\title{
ANL/ES/RP-82004
}

\section{Preliminary Investigation of Force-Reduced Superconducting Magnet Configurations for Advanced Technology Applications}

\author{
J.X. Boulllard
}

\author{
Argonne National Laboratory \\ Energy Systems Division
}

December 1992

DISCLAIMER

This report was prepared as an account of work sponsored by an agency of the United States Government. Neither the United States Government nor any agency thereof, nor any of their employees, makes any warranty, express or implied, or assumes any legal liability or responsibility for the accuracy, completeness, or usefulness of any information, apparatus, product, or process disclosed, or represents that its use would not infringe privately owned rights. Reference herein to any specific commercial product, process, or service by trade name, trademark, manufacturer, or otherwise does not necessarily constitute or imply its endorsement, recommendation, or favoring by the United States Government or any agency thereof. The views and opinions of authors expressed herein do not necessarily state or reflect those of the United States Government or any agency thereof. 


\section{Table of Contents}

Page No.

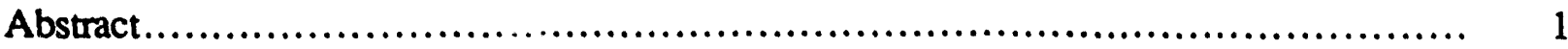

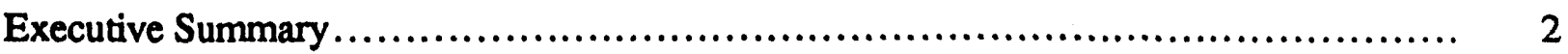

1. Background and Modifications................................................ 4

2. Introduction.................................................................... 6

2a. Review of Force-Free Solutions.................................................... 6

2b. Review of Virial Theorem/Scaling Laws ..................................... 17

3. Three-Dimensional Finite Element Computer Modeling .............................. 24

3a. Description of the TOSCA Numerical Algorithm................................. 24

4. Preliminary Comparative Study of Force-Reduced Magnets with

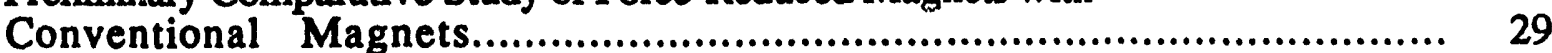

4a. Review of Proposed MHD Thruster Configurations.................................. 29

4b. Torsatron Analysis ....................................................... 33

4c. Preliminary Comparison of Electromagnetic Forces Between

Torsatron and Conventional Systems.................................... 35

4d. Comparative Study of Toroidal Pod and Toroidal Annulus Conventional

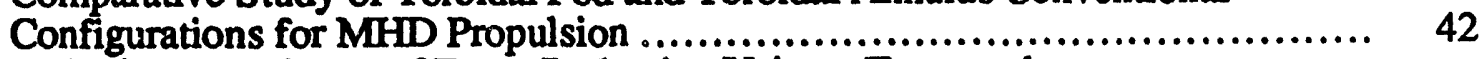

4e. Preliminary Estimate of Force-Reduction Using a Truncated Force-Free Field Geometry .................................................. 49

5. Needs and Directions for Future R\&D .................................... 70

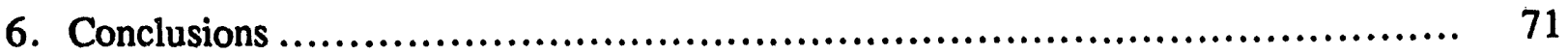

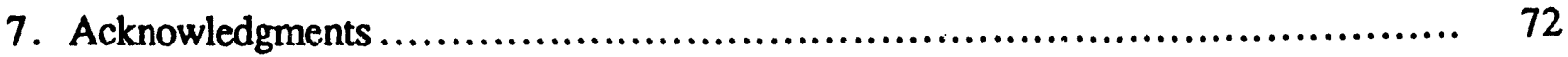

Appendices

A. Review of Superconducting Magnet Procedures ................................. 73

B. Basic Concepts in Magnetostatics .................................................. 81 


\title{
Preliminary Investigation of Force-Reduced Superconducting Magnet Configurations for Advanced Technology Applications
}

\author{
J.X. Bouillard
}

\section{Abstract}

The feasibility of new high-field low specific weight superconducting magnet designs using force-free fields is being explored analytically and numerically. This report attempts to assess the technical viability of force-free field concepts to produce high-field, low specific weight and large bore volume magnets, which could promote the use of high temperature superconductors. Several force-free/force-reduced magnet configurations are first reviewed, then discussed and assessed. Force-free magnetic fields, fields for which the current flows parallel to the field, have well-known mathematical solutions extending upon infinite domains. These solutions, however, are no longer force-free everywhere for finite geometries. In this preliminary study, force-free solutions such as the Lundquist solutions truncated to a size where the internal field of the coil matches an externally cylindrical magnetic field (also called a Lundquist coil) are numerically modeled and explored. Significant force-reduction for such coils was calculated, which may have some importance for the design of lighter toroidal magnets used in thermonuclear fusion power generation, superconducting magnetic energy storage (SMES), and mobile MHD power generation and propulsion. 


\section{Executive Summary}

In past years, it has buen acknowledged and documented that the state-of-the-art superconductor magnet technology is being too conservative in the design of magnet structure. The weight of these structures often exceed the limit imposed by the virial theorem. It is possible that present magnet design could significantly be improved by using force-free fields. This approach could open new possibilities for manufacturing large high-field magnets of low specific weight which are critical to the development of applications such as thermonuclear power stations, SMES, and mobile MHD generation or propulsion systems.

The design of force-reduced magnets is based on solutions to the force-free magnetic field equation, where the current and the field lines are parallel, thereby canceling Lorentz forces. For finite-force-free magnetic field configurations, the virial theorem sets limits on the structural mass required to contain the magnetic energy. While Lorentz forces may be eliminated in the interior of a given force-free field region, they are actually displaced on the periphery of the region where they can thus be compensated by structural tensile forces. Furthermore, it has been shown that the critical current density could be raised by as much as three times when the current flows parallel to the field lines. Therefore, potential improvements for large high-field magnets of low specific weight need to be explored and assessed, which is precisely the objective of this study.

This objective was reached by first reviewing the principal force-free winding configurations published to date, and identifying the possible technological applications of such windings. The three-dimensional finite element computer code (TOSCA) was then used to help determine magnetic field distributions and assess electromagnetic stress loads for proposed forcereduced winding configurations. Two force-reduced configurations were computationally studied: the rectangular torsatron and the truncated Lundquist coil. For these two configurations a force reduction was successfully computed in comparison with conventional windings. For the truncated Lundquist coil, the predicted solution grossly followed the theoretical Lundquist solution in the interior region of the windings, which reinforced our confidence in the computer model predictions. A force-reduction of about $25 \%$ was computed for a discrete Lundquist coil made of six helices.

The computer code was initially installed on the ANL VAX 8700, which turned out to be too slow for these types of computations. The code was then transferred and implemented on the ANL CRAY XMP supercomputer to expedite these computations. A significant effort was expended in implementing this code on both machines. 
Although the three-dimensional modeling of discrete force-free field configurations successfully showed a reduction of forces on the windings, further research needs to be conducted to study more complex coil geometries to possibly obtain greater force reductions. An optimum is to be sought which balances the structural weight reductions obtained from force-reduced designs with the increased complexity of windings, structure and cooling systems required to support these new types of windings. 


\section{Background and Motivations}

In past years, there has been considerable interest in the development force reduced superconducting magnets for a wide variety of technological applications for which high magnetic fields are needed. Coils in such high magnetic fields are subjected to large electromagnetic stresses which are usually contained by using heavy superstructures. By eliminating these electromagnetic stresses by using force-free field concepts, one could expect to significantly reduce the weight of large field magnets. Civilian and military technologies that could benefit from such concepts are briefly reviewed.

One of the most popular superconducting magnet applications is the Magnetic Resonance Imaging (MRI) technology which has now achieved the status of a mature industry. Typical magnetic fields of 1.5 to 4 Teslas with a bore space of $1 \mathrm{~m}$ are required for research and medical MRI superconducting magnets. The MRI industry significantly impacts the magnet industry, with an annual turnover greater than $\$ 600 \mathrm{M}$. MRI systems are already well developed and effectively cost-engineered. However, new designs that would reduce the weight and magnetic pollution, increase field strength, and maximize the bore space are strongly desired, especially for transportable high-field medical and research MRI systems.

Another application of high field superconducting magnets is the development of superconducting magnetic energy storage (SMES) devices. The use of these devices for electric leveling grids will only be competitive with conventional systems (pumped hydrostorage) for large units of about $10^{14} \mathrm{~J}$ or more. Since the largest magnets presently store at most 10-100 T, the use of superconducting energy storage for electric load leveling grids is only a remote possibility. Not only is the energy storage capacity of these systems an important characteristic, but equally important, is the current rise time of these systems. Figure 1 shows how the cost of energy storage varies with the size of the energy store, while Figure 2 shows distinct regions in which particular forms of energy storage are most appropriate. Capacitors are preferred for charge/discharge times below a few milliseconds, whereas flywheels, batteries, and homopolar machines are best suited for times ranging between 10 to 100 milliseconds. In the range of millisecond time rise, superconductive inductive energy storage (SMES) seems most promising. These storage devices can be used for ground and space based systems, such as electromagnetic vehicle launchers, hypervelocity guns, neutral particle beam weapons, proactive defense systems, chemical and free-electron lasers. For such devices, light weight - high energy storage capacity superconductive magnets are desired, which could offer high energy conversion efficiency, low maintenance, low capital costs and minimal production of chemical and magnetic pollution. 


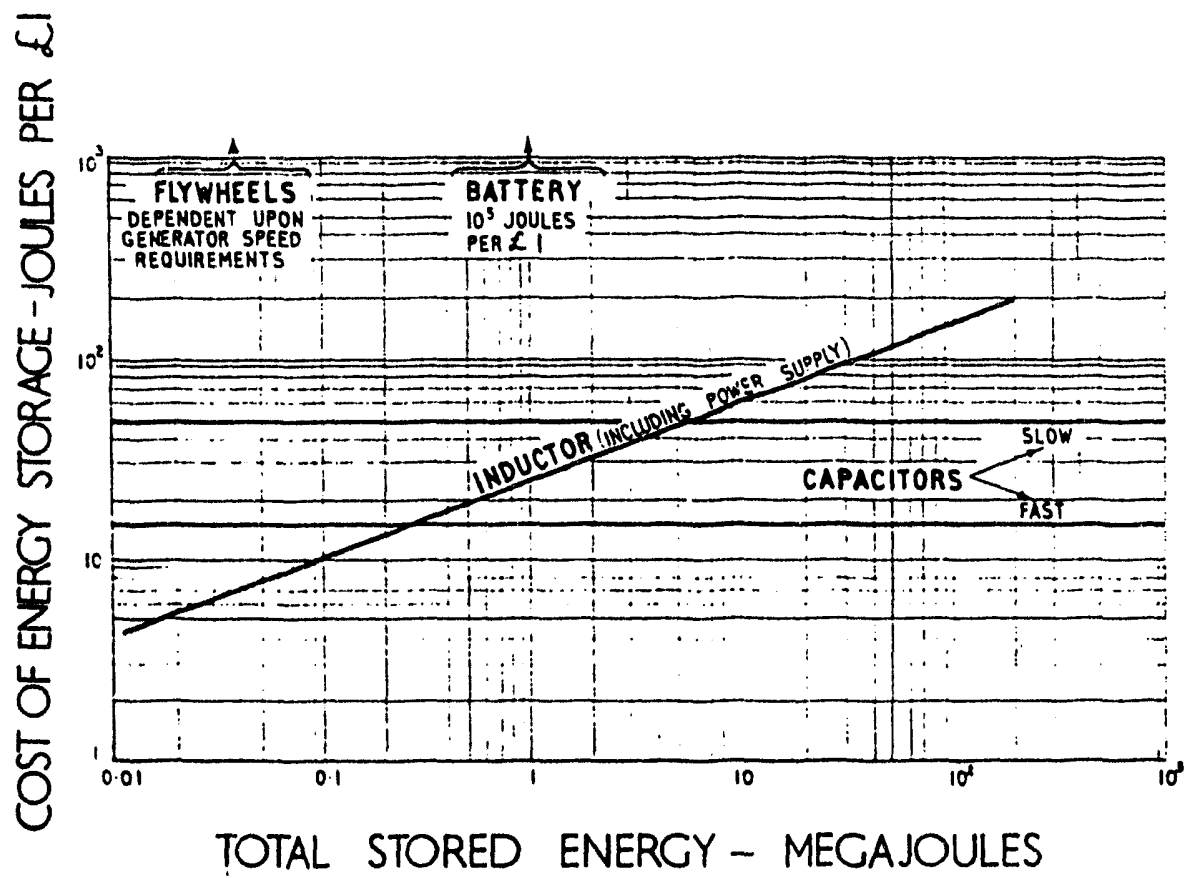

Figure 1. Cost of energy storage vs. total stored energy (Carrathers, 1961)

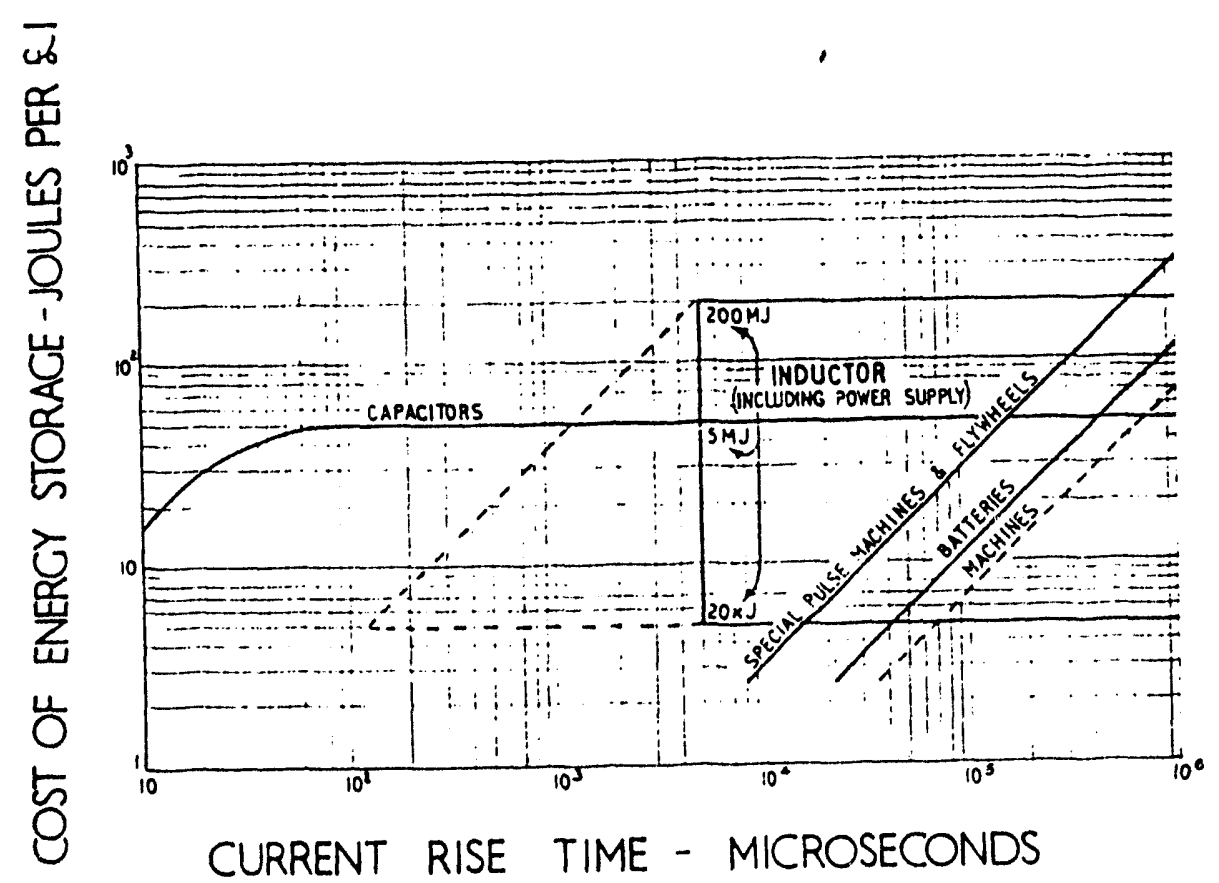

Figure 2. Cost of energy storage vs. current rise time (Carruthers, 1961) 
Finally, magnetohydrodynamic (MHD) power generation and propulsion technology would require lighter and more powerful magnets (10-12 $\mathrm{T})$ to make it competitive with present technology. This requirement becomes even more stringent for mobile generators or thrusters to be implemented on marine ships, rockets, space platforms or laboratories. Similar requirements also apply for large fusion magnets. For these toroidal magnets, the large forces and bendings moments acting on such coil systems have been partly accommodated by adopting $\mathrm{D}$-shape toroidal coils. Such coils, however, are being subjected to greater constraints than those encountered in MHD power generation or propulsion, namely they have to be stable in the presence of large pulsed fields, offer fast safety discharge performance, and permit sufficient access to the blanket and the first wall so that maintenance and replacement can be made periodically and in a timely manner. Hence, the use of force-free magnets appears to be more promising for MHD applications than for thermonuclear fusion applications, because of lesser rigid constraints imposed on the coils.

In summary, it is clear that force reduced superconducting magnets could have significant technical and economical impacts on each of these technological fields. All the application fields described above could thus provide the necessary impetus to develop a strong and well-structured research program geared to assess the technical viability and feasibility of such concepts; it is in this spirit that this report attempts to investigate and assess the technical potential for some limited force-reduced coil configurations.

\section{Introduction}

In the construction of large magnetic field superconducting coils, challenging mechanical issues have to be resolved. These issues arise because of the existence of strong forces and bending moments which become increasingly important at higher fields. These forces,could in principle be minimized through the application of force-free field concepts.

\section{2a) Review of Force-Free Solutions}

Force-free coils are coils in which the field and current are everywhere parallel. The construction of such coils with force-free conditions applied everywhere is practically not possible. However, near force-free or force-reduced regions in the coil are possible: it is, for example, possible to design a force-reduced torus coil surrounded by a force-bearing ring, as displayed in Figure 3 (Wells and Mills, 1961). 
There is renewed interest in force-reduced magnetic field configurations due to the recent discovery of high-temperature superconductors. Because the materiais that exhibit such behavior tend to be quite brittle, it is important to reduce the forces they might experience if they are to be used for high-field applications. In addition, Furth, et al. (1988) has raised the possibility that force-free field configurations may have the potential to raise the critical magnetic field and currentdensity limits in such superconductors, thus making such concepts even more attractive.

For finite force-free magnetic field configurations the virial theorem, which can be used to relate the stored magnetic energy of the system to the integral of the trace of the stress tensor over the magnetic field volume, sets structural limits that must be obeyed in practice. While stresses may be eliminated in a given region, they cannot be canceled everywhere. In general, magnetic stresses have to be contained at the periphery of the coil region.

Several force-free configurations have already been studied. The simplest configuration is the one given by Bobrova and Syrouastiki (1979) for a planar constant- $\alpha$ force-free configuration. The field is given by

$$
B=(\operatorname{Cos} \alpha z, \operatorname{Sin} \alpha z, 0)
$$

in the infinite region between the fixed boundaries $z=0$ and $z=1$, as shown in Figure 4 . The current density, $j=$ curl $B$, is given by:

$$
j=(-\alpha \operatorname{Cos} \alpha z,-\alpha \operatorname{Sin} \alpha z, 0)=-\alpha B
$$

which makes this configuration a force-free configuration since JXB $=0$. Forces are only generated at the periphery of the device. Note that the periphery of the device could be made circular, limiting the angle stresses. In this device, the planar layers of superconducting materials could be laid on each other, thus making this configuration well suited for planar thin films of high temperature superconductors.

For cylindrically symmetric fields, Furth, et al (1957) described the following expression for constant $\alpha$ force-free solution

$$
\begin{aligned}
& \mathrm{H}_{\phi}=A \cos (k z) J_{1}\left[\left(\alpha^{2}-k^{2}\right)^{1 / 2}\right], \\
& H_{z}=A\left[\left(\alpha^{2}-k^{2}\right) 1 / 2 / \alpha\right] \cos (k z) J_{d}\left[\left(a^{2}-k^{2}\right)^{1 / 2}\right] \\
& H_{r}=A(k / \alpha) \sin (k z) J_{1}\left[\left(r\left(a^{2}-k^{2}\right)^{1 / 2}\right],\right.
\end{aligned}
$$




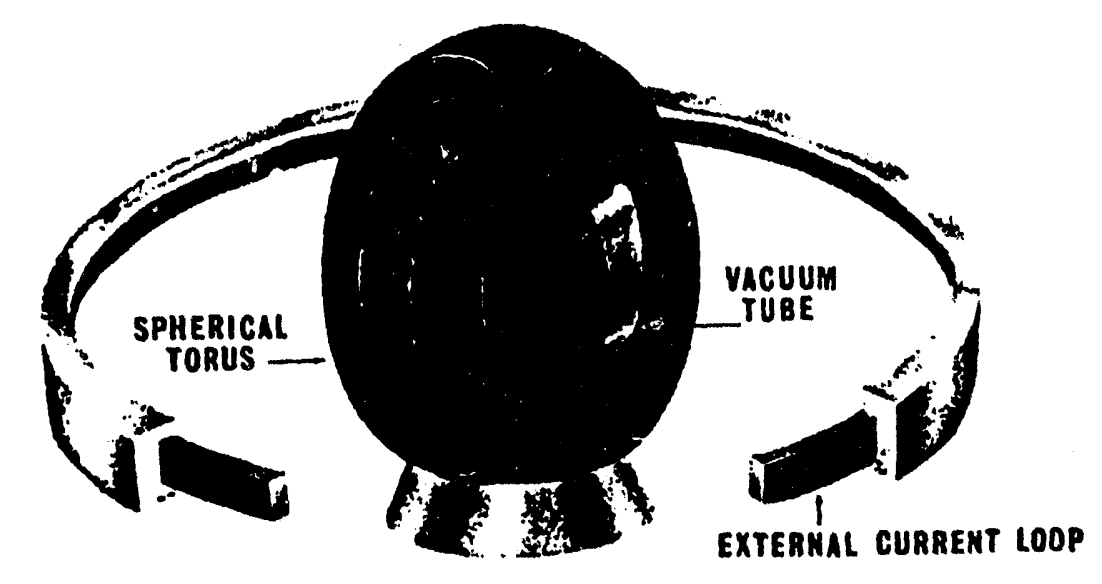

Figure 3. Schematic of the force-free torus of Wells and Mills (1961).
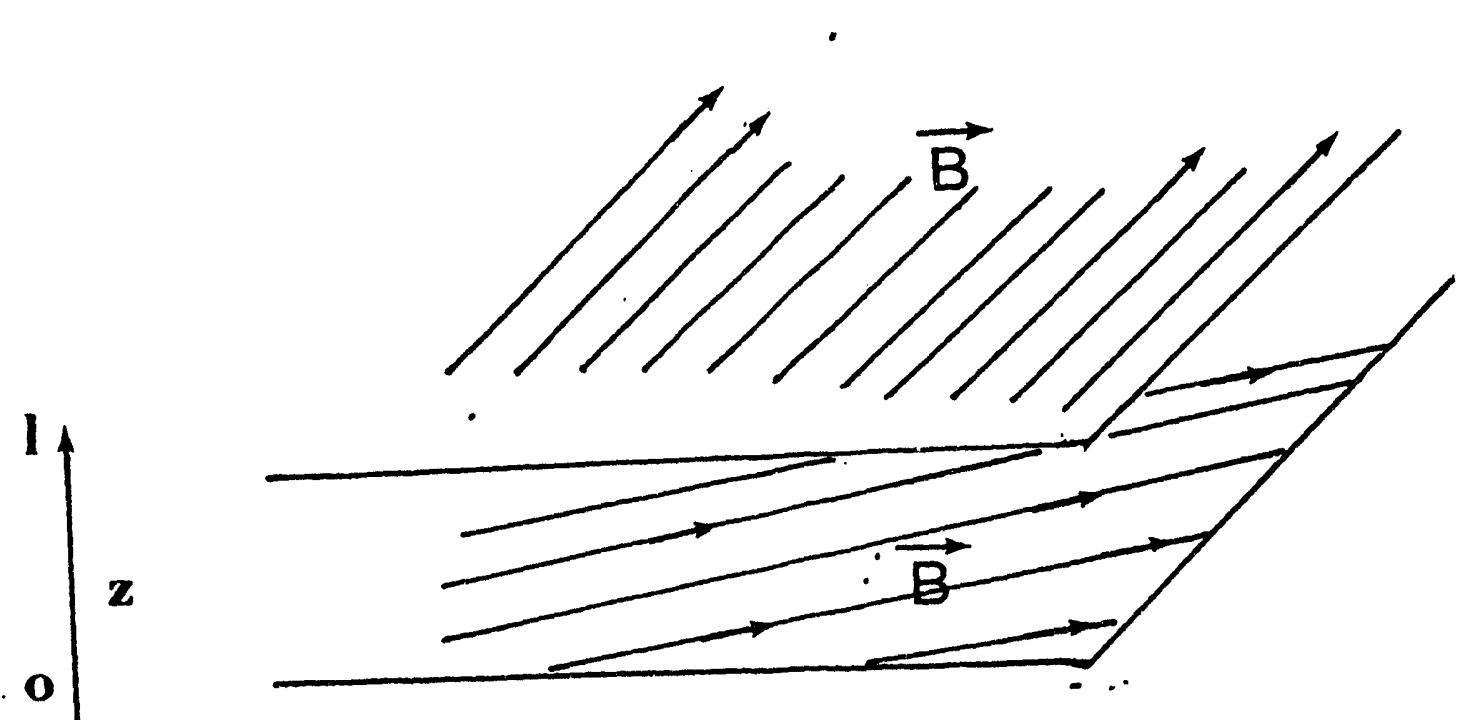

Figure 4. Planar force-free configurations (constant $\alpha$ ) 
where $A$ and $k$ are constants. For $k=0$, the radial component vanishes and the solution becomes that of Lundquist (1951). The field lines of the Lundquist solution are shown in Figure 5. For nonconstant $\alpha$ cylindrically symmetric solutions, Marsh derived a set of solutions that are presented in Figure 6 (Marsh, 1990). For axially symmetrical spherical nonconstant $\alpha$ solutions, Marsh further generalized the Grad-Shafranov equations for which the magnetic field components are expressed in terms of the flux function. This resulted in a form of Bernouilli's equation for the function $\alpha$ and an equation for the flux equation which depends on an arbitrary function of the flux. Typical results of nonconstant alpha force-free solutions are shown in Figures 7 and 8 . As can be seen in Figure 8, the magnetic surfaces are generally toroids with banana-like crosssections.

For toroidal coordinates, Buck (1965) derived the constant $\alpha$ force-free solution which is illustrated in Figure 9. Tsujii (1991) generalized Buck's solution for axisymmetric torus of arbitrary ratios in form of infinite series. Though great strides have been made in establishing analytical solutions of force-free fields for various geometries, the application of these solutions to produce useful force-reduced magnets has been slow, if not nonexistent. Although very promising, the theoretical solutions which often times assume some perfect symmetry do not easily lend themselves to the practical manufacturing of magnets. Several practical reasons explaining the difficulty of using such concepts to manufacture force-reduced coils may be given as: a) force-free fields in essence are very nonuniform and, therefore, of limited use, b) coil winding is complex and thus difficult to manufacture, $c$ ) maintenance, replacement of such coils could be difficult and costly, and d) coils of finite-size cannot be strictly force-free. The last reason is a critical one, and brings the question of how much structure reduction can we expect to gain using force-free mathematical solutions truncated to a limited and finite geometrical domain? Such questions will be explored in this report for a truncated Lundquist coil.

Several attempts in this direction have been made to produce force-reduced configurations. One of these configurations, shown in Figure 10, has been proposed by Mawardi (1975). In this configuration the poloidal and toroidal components have been decomposed by setting a coil carrying a poloidal current inside a toroidal shell which supports coils carrying a toroidal current. Of course, this arrangement is not purely force-free, but can be said to be "force compensated" or "force-reduced". The poloidal currents form a toroidal magnetic field which is completely contained inside the torus. The direction of the mechanical forces on the torus are compressive for the toroidal current sheet and expansive for the poloidal current sheet. Mawardi estimated the cost savings realized when force-free coils are used instead of conventional magnets for superconducting magnetic energy storage applications as indicated in Tables 1 (a) and (b). His 


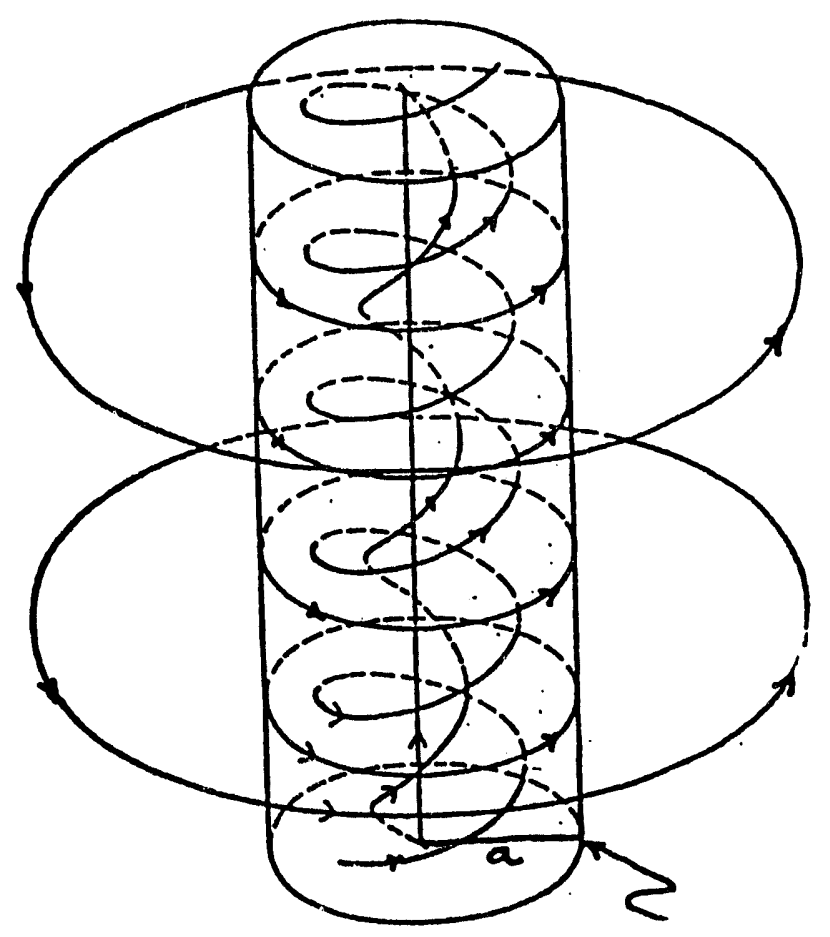

Figure 5. Force-free cylindrical solution (Lundquist) 


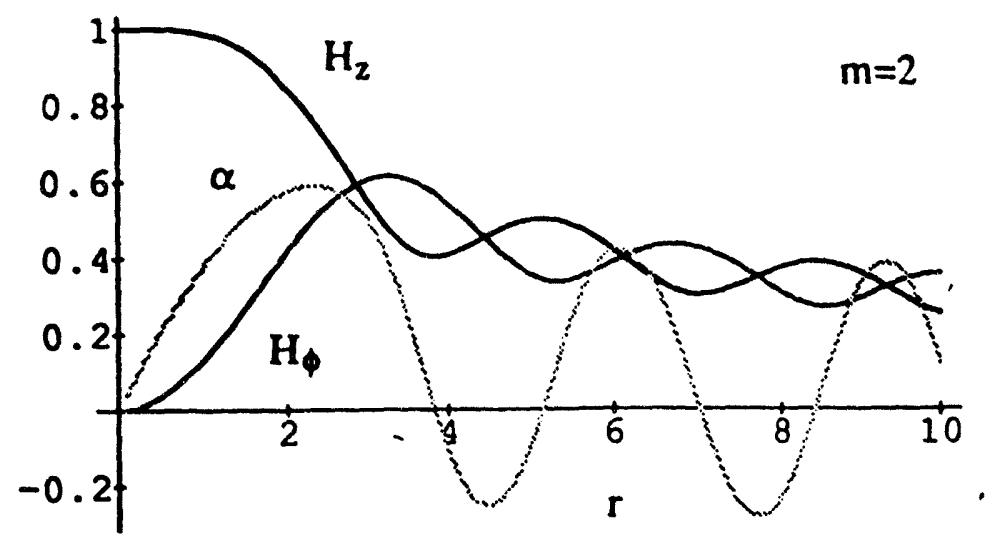

Figure 6. Magnetic field components $\mathrm{Hz}(r), \mathrm{H}_{\phi}(r)$, and the function $\alpha(r)$ for $\mathrm{m}=$ 2.(Marsh, 1990)
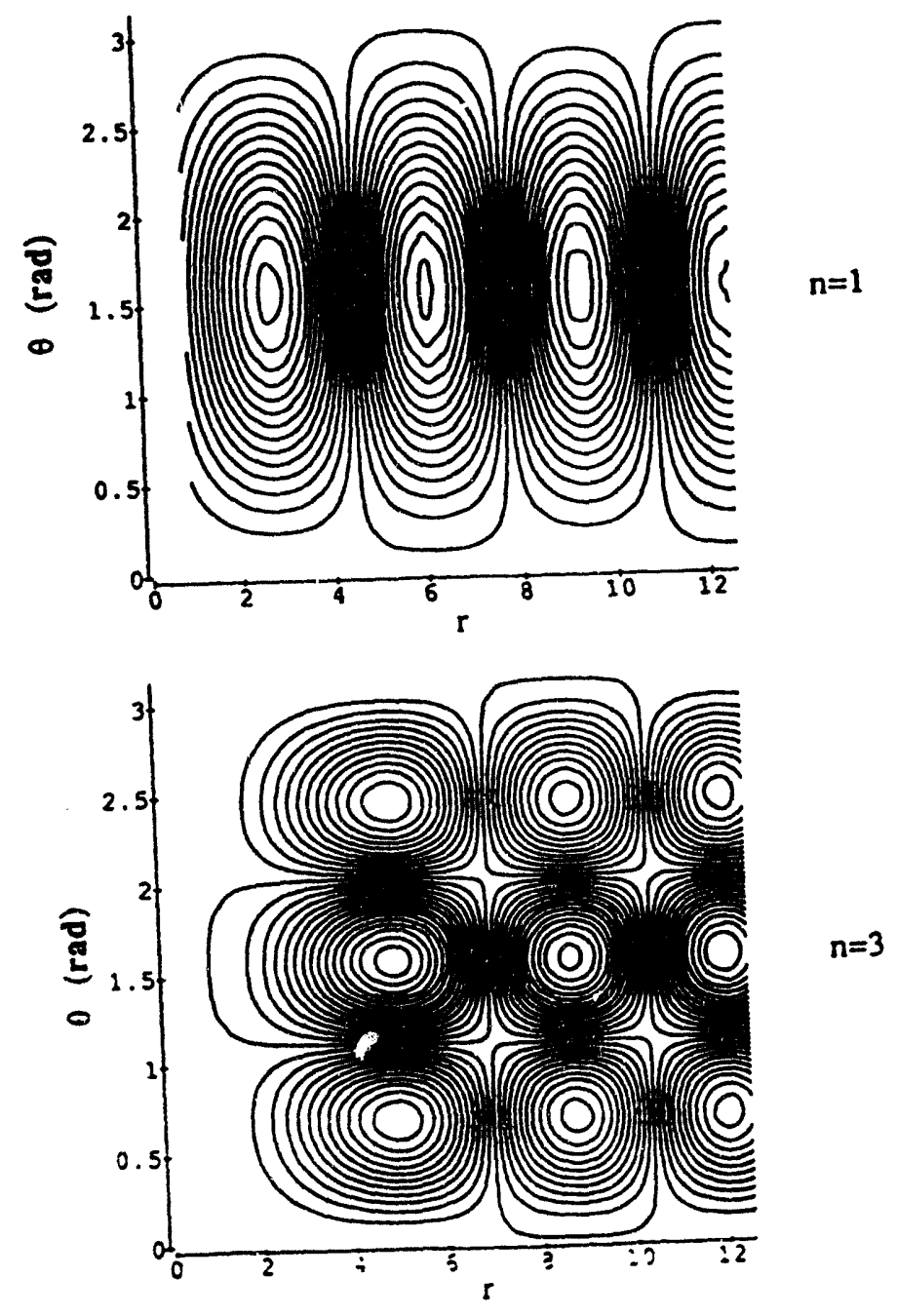

Figure 7. Contour Plot of $\psi=\sqrt{\pi / 2 \pi} J_{n+1 / 2}(r)(1-\mu) P_{n}^{(1,-1)}(\mu)$ for $n=1$ and 3, (Marsh, 1992) 


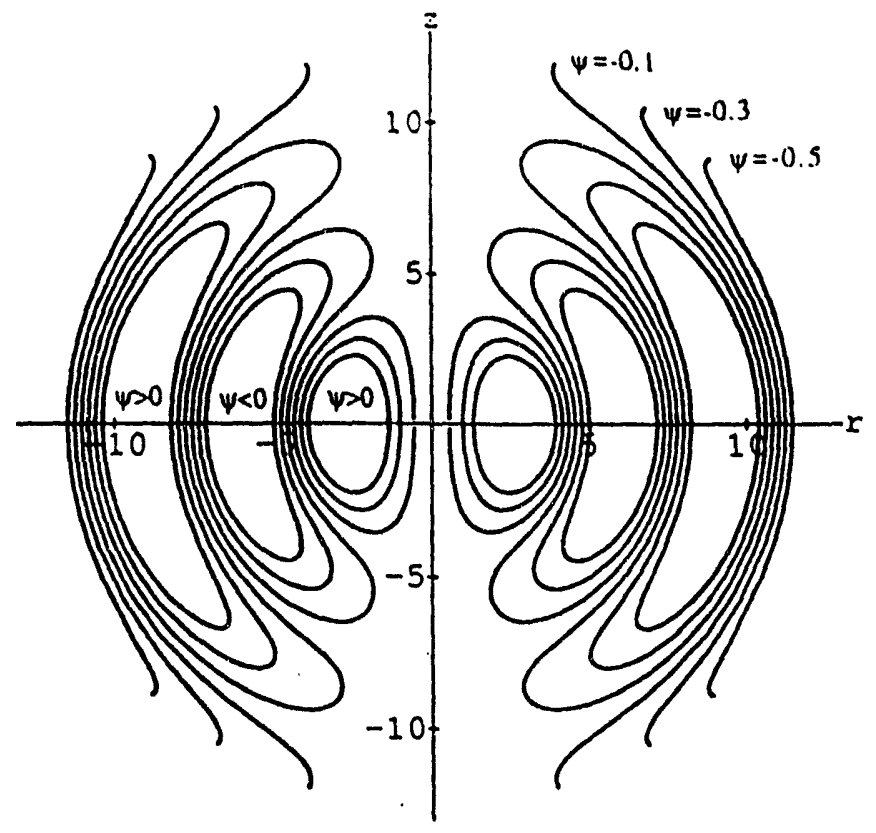

Figure 8. Parametric plot of $\psi=\pi \sqrt{\pi / 2 r} J_{n}+1 / 2(r)(1-\mu) P_{n}^{(1,-1)}(\mu)$ for $\psi .= \pm 0.1, \pm 0.3, \pm 0.5$. Note that $\psi=0$ on the $z$ axis.(Marsh, 1992).

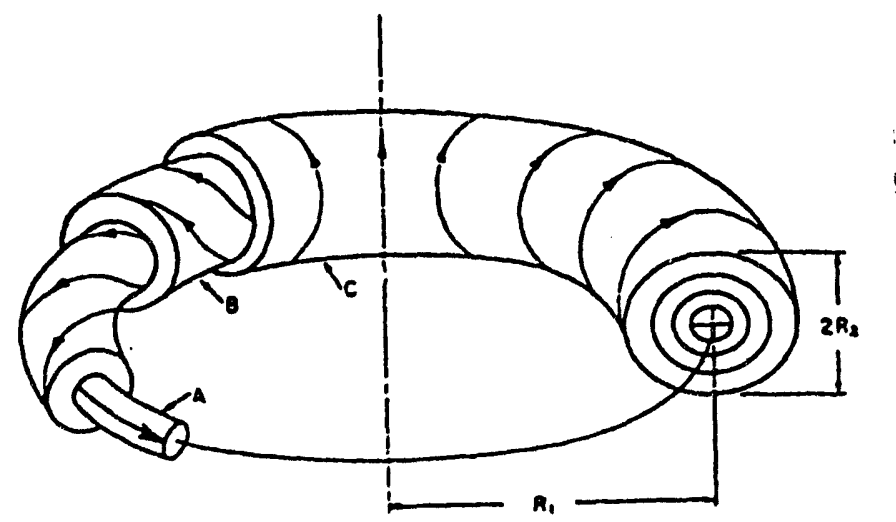

Figure 9. Typical constant $\alpha$ force-free solution for toroidal geometry (Buck, 1965) 


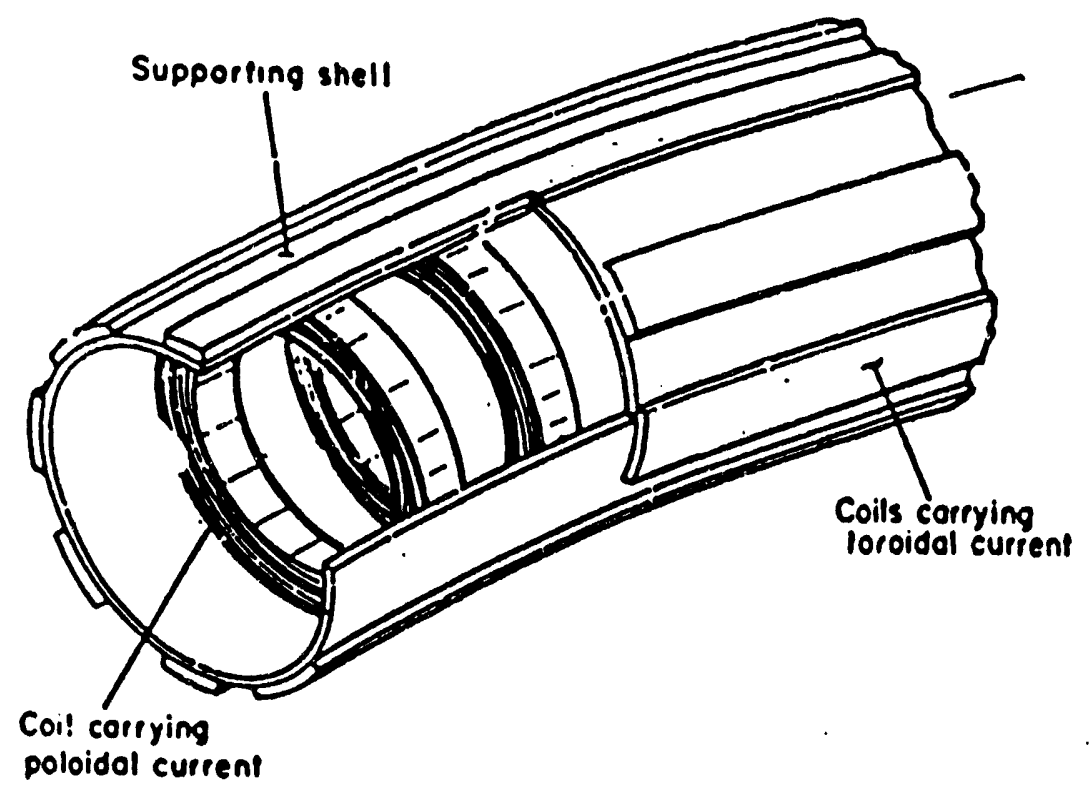

Figure 10. Reduced force energy storage concept. The poloidal coils produce outward forces which are in opposition to the inward forces of the toroidal coils.(Mawardi, 1975). 


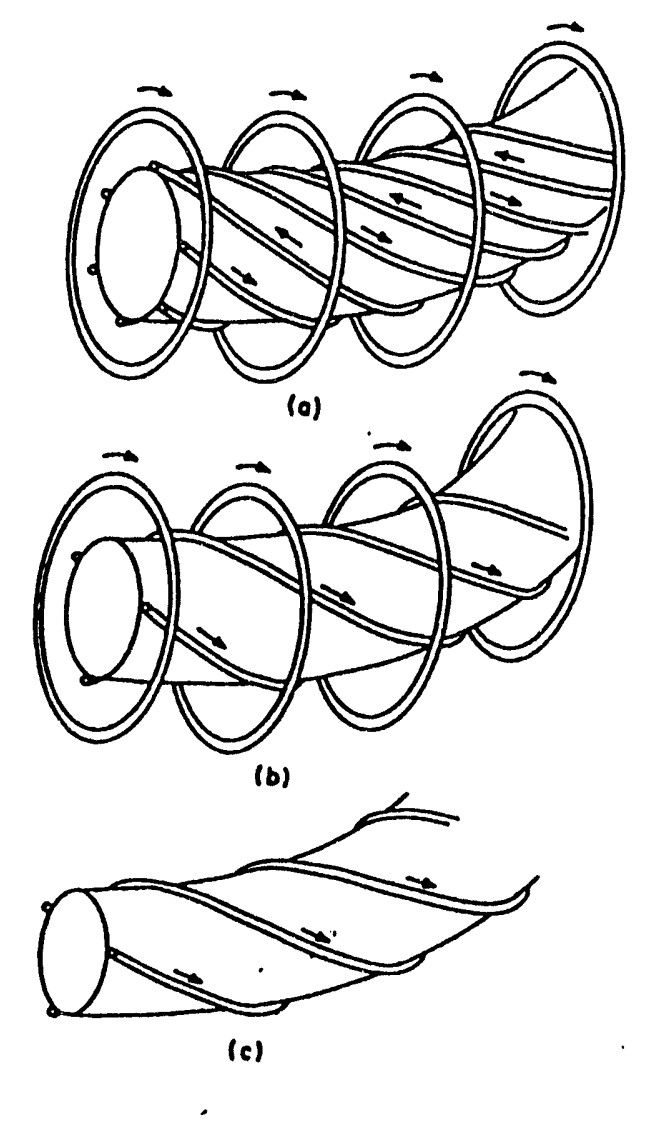

Figure 11. Schematic diagram of (a) stellarator, (b) heliotron, and (c) torsatron windings.(R. Thome, 1982) 
- Table 1 (a)

Cost Estimates for Force-Free Coil* (1975 dollars)

\begin{tabular}{|c|c|c|c|c|}
\hline Energy Stored (MJ) & $3 \times 10^{4}$ & $10^{6}$ & $3.0 \times 10^{7}$ & $10^{8}$ \\
\hline Cost of superconductor $\left(\$ \times 10^{6}\right)$ & 2.24 & 22.77 & 261.96 & 489.7 \\
\hline Dewar cost $\left(\$ \times 10^{6}\right)$ & 2.0 & 24.6 & 300 & 581 \\
\hline Structure $\left(\$ \times 10^{6}\right)$ & 0.267 & 8.67 & 338 & 866.67 \\
\hline ac-dc converter $\left(\$ \times 10^{6}\right)$ & 0.04 & 1.4 & 54 & 140 \\
\hline Miscellaneous $\left(\$ \times 10^{6}\right)$ & 47 & 2.1 & 51 & 128 \\
\hline Total & 5.02 & 59.24 & 51 & 2205.37 \\
\hline
\end{tabular}

Table 1 (b)

Comparison of Construction Costs of Force-Free Coil

with Costs of Cylindrical Coil* (1975 dollars)

\begin{tabular}{lllll}
\multicolumn{1}{r}{ Energy Stored $(\mathrm{MJ})$} & $3 \times 10^{4}$ & $10^{6}$ & $3.0 \times 10^{7}$ & $10^{8}$ \\
\hline Force-free coil $\left(\$ 10^{6}\right)$ & 5.02 & 59.54 & 1004.96 & 2205.37 \\
Conventional coil $\left(\$ 10^{6}\right)$ & 5.2 & 73.4 & 1641 & 3864 \\
*Mawardi (1975) & & & &
\end{tabular}


analysis revealed that no significant gains are made at low energy storage, but beyond $1 \mathrm{MJ}$, substantial gains $(40 \%)$ could be achieved with force-free coils.

Other magnet geometries such as the stellarator, heliotron and torsatrons have been proposed for thermonuclear fusion power generation. These magnets use helical windings of different helicity, as shown in Figure 11. The stellarator consists of 2-1 helical wires with alternating direction of current, but large forces exist on the helical coils. By eliminating the appropriate set of alternate helical coils, the remaining coil set experiences no outward forces, and this geometry is known as the heliotron. By removing the toroidal coils, one obtains a simpler geometry called the torsatron. By appropriately choosing the pitch of the windings, the torsatron configuration can be made essentially free of external forces, thereby requiring less structural material to support it.

Recent inventions by Prueitt, et al. have been geared towards establishing several configurations of conductors capable of supporting extremely high magnetic fields suitable for plasma confinements. Typical wire arrangement proposed by Prueitt, et al. is shown in Figure 12, with the corresponding isomagnetic field lines shown in Figure 13 (Prueitt, et al., 1991). In this arrangement, the wires transport currents of opposed directions, thus canceling the large electromagnetic forces. This arrangement is based on finding low-force configurations of electric current carrying wires, which have been shown to be equivalent to locate stationary states for flowing systems having localized logarithmic potentials. Campbell and Kadtke (1987a) and Kadtke Campbell (1987b) showed that equilibrium (force-free) configurations could be possible in some instances.

\section{2b) Review of the Virial Theorem/Scaling Laws}

Two puzzling ideas have intrigued many magnet designers regarding the structural mass necessary to hold magnetic forces. One is, of course, the force-free concept, while the other is the limits given by the virial theorem. Superficially, if a magnet is designed according to the force-free concept, $\mathrm{JXB}=0$, thus one could expect that there is no need for structural force to hold the magnetic field in place. On the other hand, the virial theorem sets a lower limit of structural material necessary to hold the coils/field together. This dilemma is quickly resolved by realizing that although volumetric forces are eliminated in a force-free concept, surface forces at the coil periphery can be present. Let us review the virial theorem, and see how these two seemingly contradicting hypotheses are in fact complementary. 


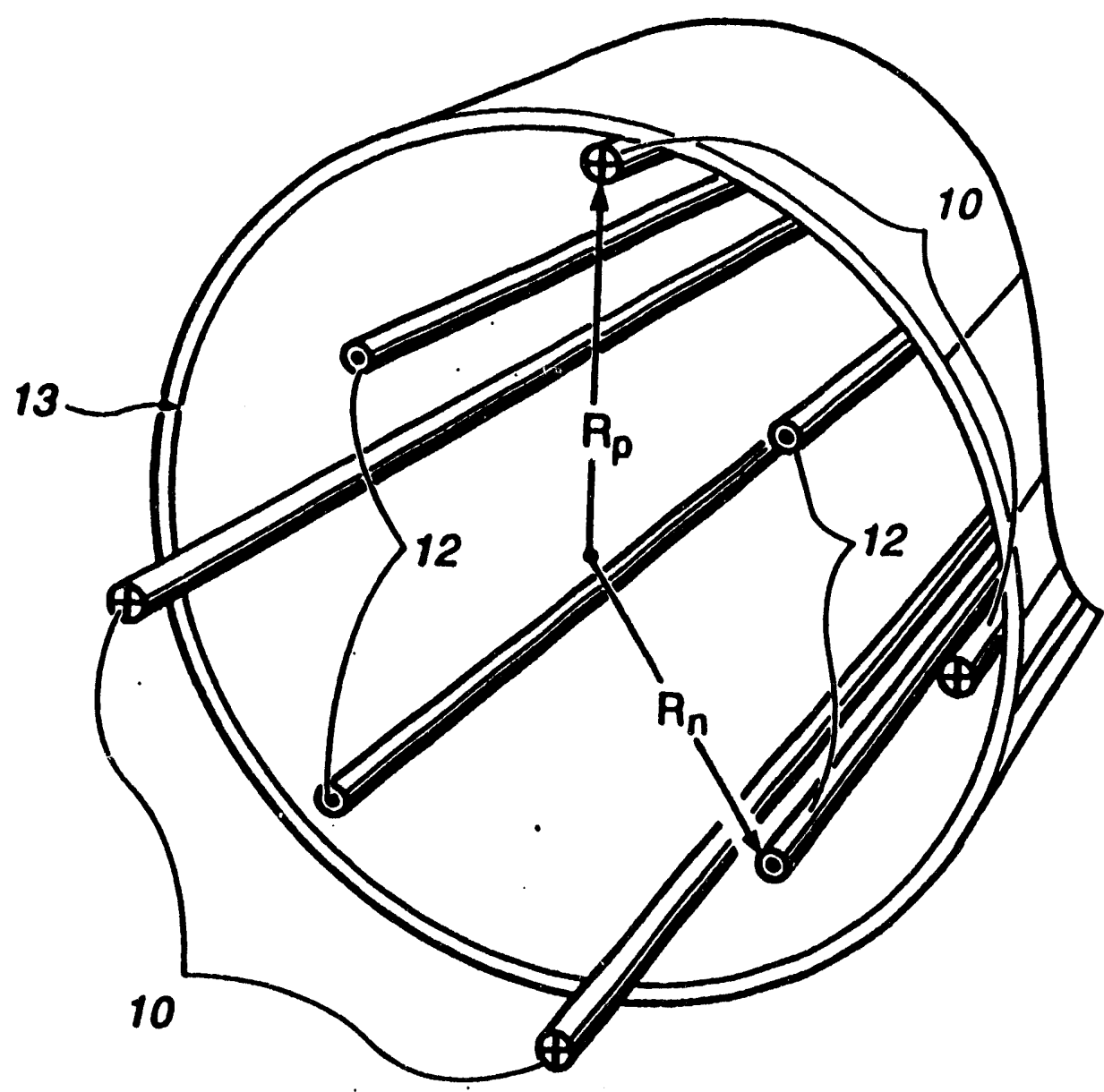

Figure 12. Schematic representation of a force-reduced configuration of eight conductors (Prueitt, et al., 1991). 


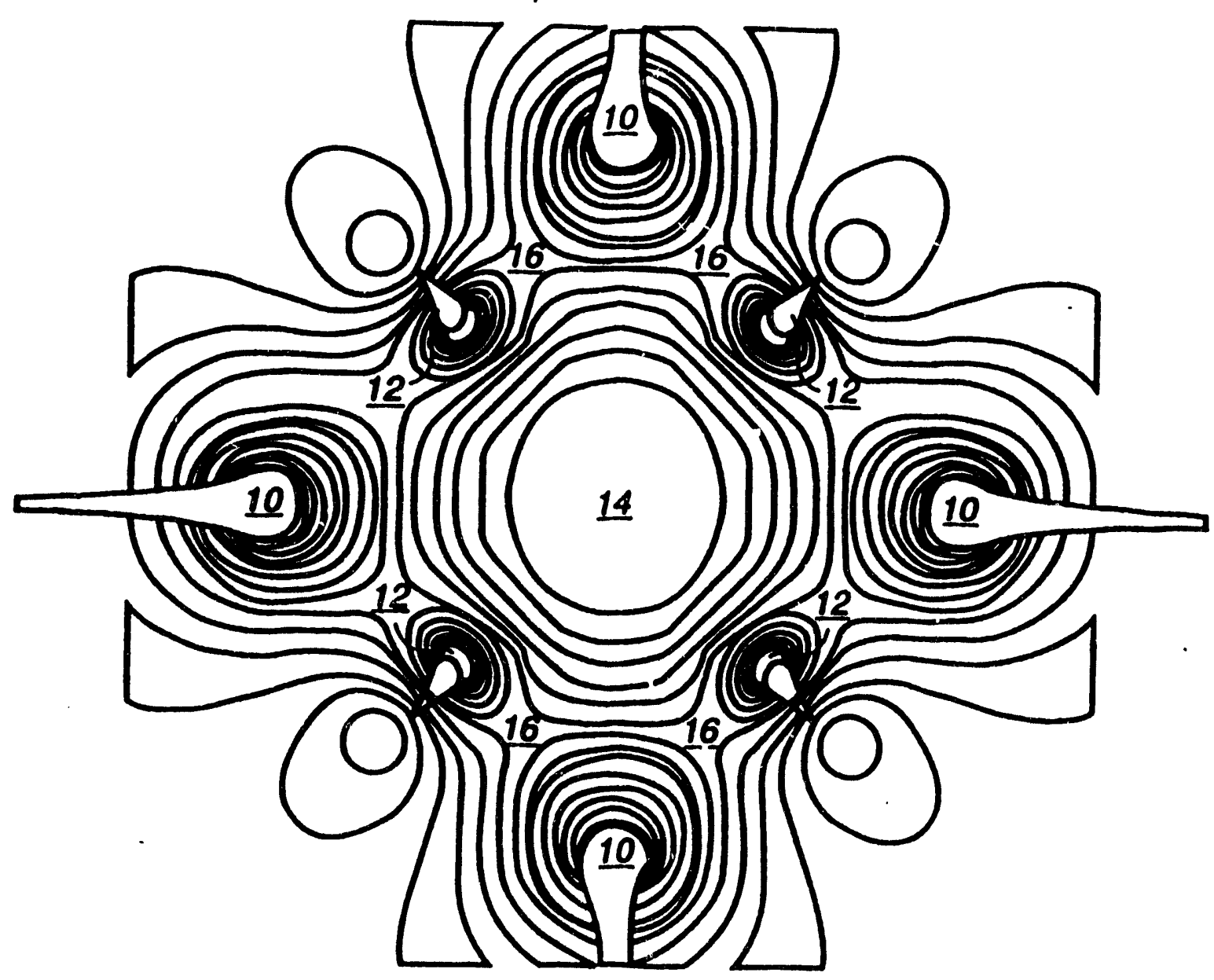

Figure 13 Representation of the strength of the magnetic field with isofield contours for the system shown in Figure 12 (Prueitt, et al., 1991). 
The virial theorem of Clausius can be extended by analogy to the case of a coil and its magnetic field. When the electromagnetic forces are in equilibrium with elastic forces in a medium that contains the electromagnetic field, then the Maxwell stress tensor $T$ and the elastic stress tensors $\mathrm{S}$ obey the following relationship:

$$
\nabla \cdot(\mathrm{T}+\mathrm{S})=0 \text {. }
$$

With the summation convention, this equation can be written as

$$
\frac{\partial}{\partial x_{j}}\left(T_{i j}+S_{i j}\right)=0
$$

Forming the inner product of the coordinate vector $x_{j}$ with this equation and integrating over a volume $\mathrm{V}$ bounded by a surface $\mathrm{S}$, we have

$$
\int_{v} x_{i} \frac{\partial}{\partial x_{j}}\left(T_{i j}+S_{i j}\right) d v=0
$$

Integrating by parts and applying Gauss's theorem, one obtains

$$
\int_{S} n_{j} x_{i}\left(T_{i j}+S_{i j}\right) d A-\int_{v}\left(T_{i i}+S_{i i}\right) d v=0 .
$$

where the Maxwell stress tensor, $T$, is defined as

$$
\begin{aligned}
& T_{i j}=\frac{\sigma_{i j}}{2 \mu_{0}} B^{2}-\frac{B_{i} B_{j}}{\mu_{0}} \\
& \int_{S} n_{j} x_{i}\left(T_{i j}+S_{i j}\right) d A-\int_{v}\left(T_{i i}+S_{i i}\right) d v=0 .
\end{aligned}
$$

The trace $T_{i i}$ of the magnetic stress tensor is $+\frac{B^{2}}{2 \mu_{0}}$. Thus

$$
\int_{v}\left(+\frac{B^{2}}{2 \mu_{0}}+S_{i i}\right) d v=\int_{s} n_{j} x_{i}\left(T_{i j}+S_{i j}\right) d A .
$$

This equation is known as the virial theorem. 
If the surface integral converges to zero at infinity, thus we have

$$
-\int_{v} S_{i i} d V=\int_{v} \frac{B^{2}}{2 \mu_{0}} d V
$$

which indicates that all the electromagnetic energy stored has to be compensated by some structural elastic energy. Note at this point, no assumption is made on the form of the field. The elastic forces are tensile in character because of the sign of the left-hand side of the equation.

If the surface integral is not zero, it is positive because of the definite positive form of the integrand. Therefore, if all the magnetic energy is not compensated by a volumetric tensile stress, it has to be confined by surface forces on the surface $S$. Hence, the tensile forces are only exerted on the periphery of the domain. This is typically the case for force-free fields, since in the volume under consideration, no volumetric forces are present. All the magnetic forces created in the volume must be compensated by tensile forces on the surface of the volume.

The mass of the structure is given by

$$
M_{s}=\frac{\rho_{s}}{\sigma_{w}} \int_{v}\left|S_{i i}\right| d v
$$

where $\sigma_{W}$ is the working stress and $\rho_{s}$ is the density of the structure. The mass necessary to support the structure is, therefore

$$
M_{s} \geq \frac{\rho_{s} B^{2}}{2 \mu_{0} \sigma_{w}}
$$

Moon (1982) found that for the majority of the MHD and thermonuclear fusion superconducting magnets, the mass of the magnets scales as $\mathrm{E}=\mathrm{CM}^{4 / 3}$, rather than the linear scaling implied by the virial theorem, as shown in Figures 14 and 15 . He also underlined that all of the superconducting magnets he studied are heavier than the limit imposed by the virial theorem, by about an order of magnitude. He concludes his analysis by realizing that the primary constraint on the design of these magnets is not stress, but rather current density, field strength, uniformity, and thermal stability. Moon concluded that reduction of magnet weight, although desirable, may not be easily to accomplished because of the many other interdependent components and constraints involved in the construction of superconducting magnets (see Appendix A). 
As fields of the order of $20 \mathrm{~T}$ seem to be attainable with conventional low $\mathrm{Tc}$ superconducting technology, critical fields may be raised by orienting the field in the direction of the current. Under this condition, it has been observed that the critical current density can significantly be augmented, which is often expressed as (Cody, et al., 1964)

$$
\mathrm{Jc}=\alpha(\mathrm{HS} \operatorname{Sin} \theta+\mathrm{Bo})
$$

where $\theta$ is the angle between the current and the field $H$ with $\alpha$ and Bo being constants. As can be seen, if the current flows parallel to the field $\theta=0$ the maximum critical field becomes equal to $\mathrm{Jc}=\alpha /$ Bo. Furth, et al., (1988) indicate that $\mathrm{J}_{\mathrm{cl} 1}$ can be at least three times higher than for some superconductors. As a result, the use of force-reduced configuration could mean the possibility of winding magnets which could provide fields of the order of $100 \mathrm{~T}$. At these high fields, the internal conductor magnetic pressure rises quickly to the order of $100 \mathrm{M} \mathrm{Pa}$, and hoop stresses are of the order of $5000 \mathrm{M} \mathrm{Pa}$ which could introduce significant strain on these conductors during normal operation and therefore decrease the critical current density (see Appendix A). It is therefore clear that if large fields are needed the force-free concept becomes attractive since it systematically eliminates all these concerns. In fact, force-free field concepts may be the only practical way of containing such large stresses. 


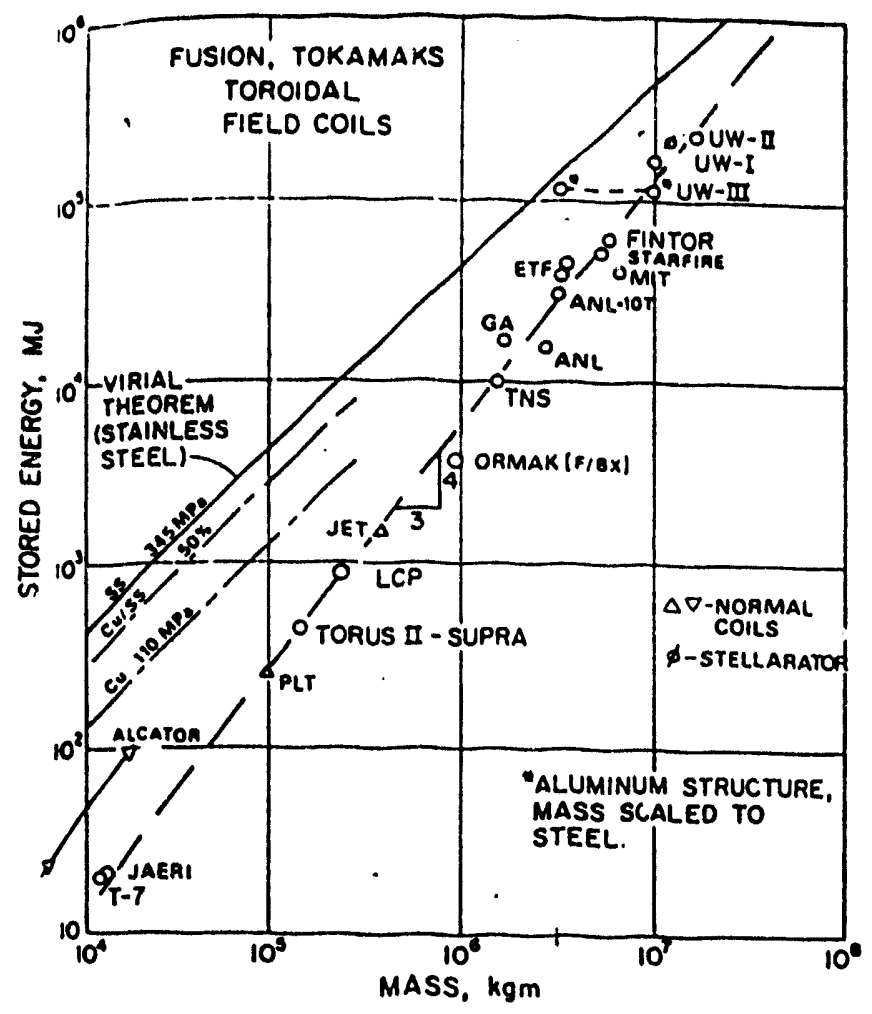

Figure 14 Stored magnetic energy vs. structural mass for tokamak toroidal field coils for fusion reactors. The virial limit is based on a design stress of $34.5 \mathrm{kN} / \mathrm{cm}^{2}(50,000 \mathrm{psi})$ in stainless steel (Moon, 1982)

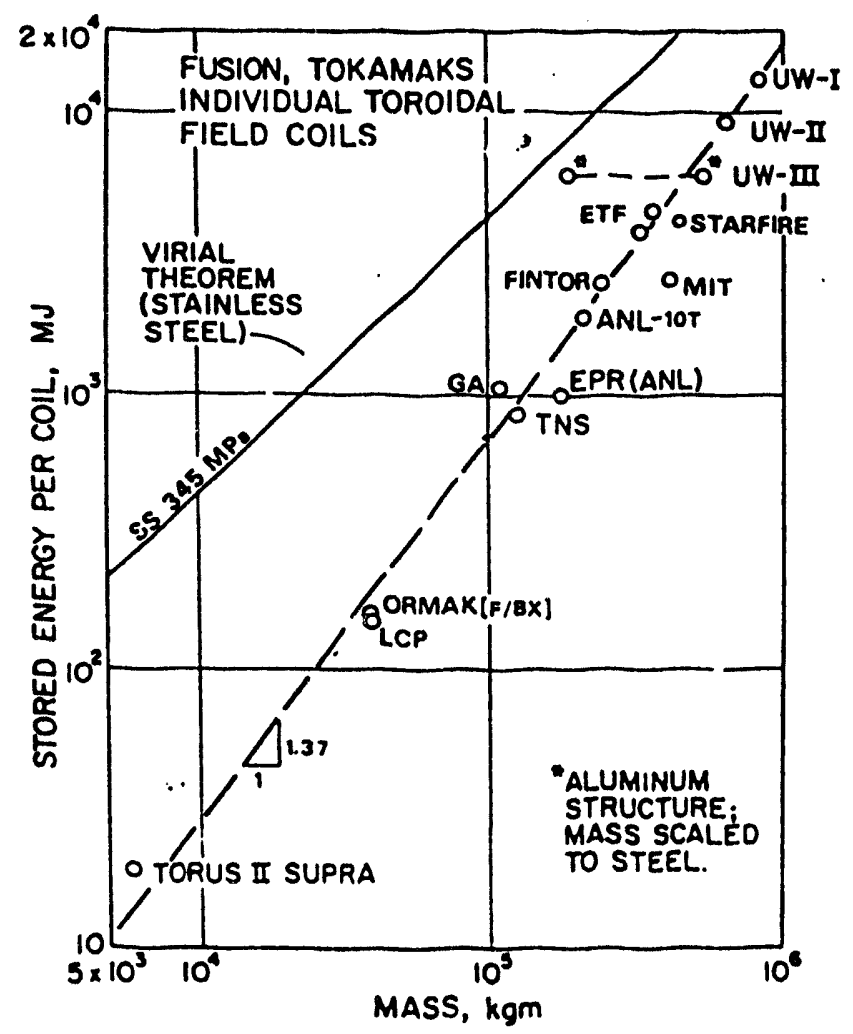

Figure 15 Stored magnetic energy vs. structural mass for individual tokamak toroidal field coils for fusion reactors. The virial limit is based on a design stress of $34.5 \mathrm{kN} / \mathrm{cm}^{2}$ (50,000 psi) in stainless steel (Moon 1982). 


\section{Three-Dimensional Finite Element Computer Modeling}

A comprehensive computer model that can address the main design issues prior to the construction of the magnet can be a very valuable and cost effective design tool. Most designs of superconducting magnets rely on computer modeling. Ideally, the computer model should calculate the magnetostatics, the structural dynamics and heat transfer performances of the magnet. However, such complexity often leads to computer programs that are highly cumbersome and inefficient to use. The magnetostatics and the structural dynamics must be computed interdependently. A typical illustration of the stress dynamics is shown in Figure 16 in the work of Chapman, et al. (1989) for the design of the yoke and collars of a supercollider dipole magnet. The computer model predicts the mechanical stresses and deformations during various cool-down sequences of the assembly. The computer code used in their analysis was the ANSYS finite element code developed by Dawson Analysis Systems. In this work, another computer code, r.amed TOSCA, was used. TOSCA only solves the three-dimensional magnetostatics but offers, however, better solution convergence performances than the ANSI computer code. As indicated in Appendix A, the final design of a superconducting magnet often relies on the magnetostatics, thermal and structural analyses, which are dependent of one another. In our analysis, however, thermal and structural effects are ignored, and only magnetostatics effects are considered. TOSCA computer ccule only solves the three-dimensional magnetostatics using a finite element algorithm. Since its solution deals only with magnetostatics, it offers better solution convergence performances than the ANSI computer code.

\section{3(a). Description of the TOSCA Numerical Algorithm}

The three-dimensional computer code TOSCA was originally developed at the Rutherford Appleton Laboratory, in England, to study magnetostatics and electrostatics problems. The code was then further extended and packaged with new graphics, pre- and post-processors by Vector Fields Limited. A copy of the computer code has been purchased by ANL.

Finite element discretization forms the basis of the TOSCA numerical algorithm, which can be used to simulate linear, nonlinear and anisotropic phenomena. In this code, the total magnetic field intensity $(\overrightarrow{\mathrm{H}})$ is decomposed into a reduced magnetic field intensity $\left(\overrightarrow{\mathrm{H}}_{m}\right)$ and a current magnetic field intensity $\left(\overrightarrow{\mathrm{H}}_{\mathbf{s}}\right)$ : 

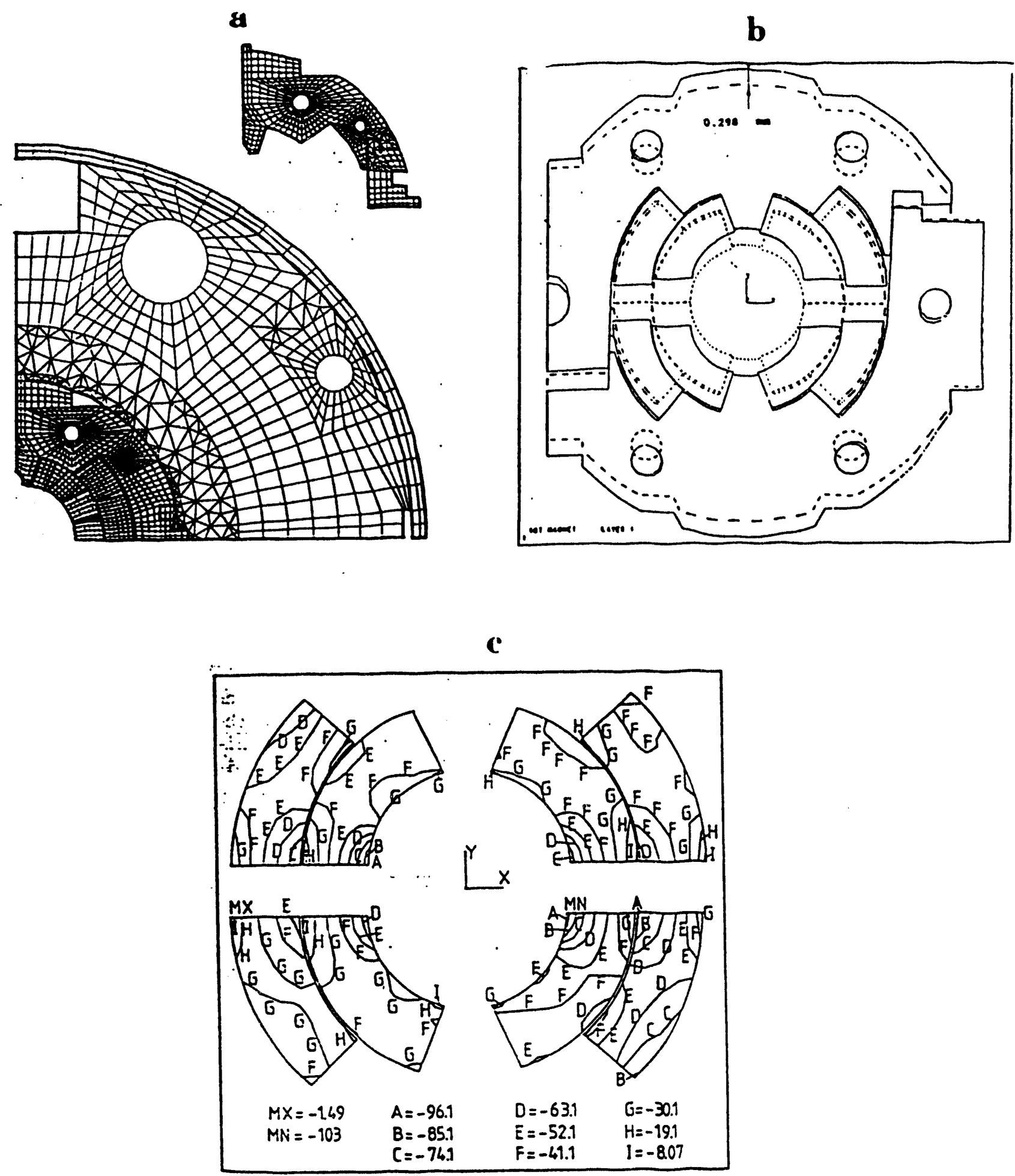

Figure 16 (a) Finite element model of a superconducting super collider dipole magnet, (b) computed deformation of the coils and collars, (c) computed stresses on coils (computations made using the ANS YS computer code (Chapman, et al. 1989). 


$$
\overrightarrow{\mathrm{H}}=\overrightarrow{\mathrm{H}}_{\mathrm{m}}+\overrightarrow{\mathrm{H}}_{\mathrm{s}}
$$

since $\left(\vec{H}_{m}\right)$ represents the magnetic field where no current sources are present,

$$
\text { curl } \overrightarrow{\mathrm{H}}_{\mathrm{m}}=0 \text { or } \overrightarrow{\mathrm{H}}_{\mathrm{m}}=\operatorname{grad} \phi
$$

where $\phi$ is the reduced scalar potential. The field due to the current sources, can be expressed by means of the Biot and Savart's Law (see derivation in Appendix B):

$$
\overrightarrow{H_{s}}=\int_{\Omega_{j}} \frac{\vec{J} \times \vec{R}}{\mid \vec{R} / 3} d \Omega_{j}
$$

In TOSCA, a quadrature is used to compute this integral. The requirement that divergence of the magnetic flux is zero, leads to the following equation:

$$
\nabla \cdot \mu \nabla \phi-\nabla \cdot \mu\left(\int_{\Omega_{j}} \frac{\vec{J} \times \vec{R}}{|\vec{R}|^{3}}\right) d \Omega_{j}=0
$$

This equation is a second order elliptical equation that can be easily solved using a finite element iterative routine. Equation (19) can be recast as

$$
\nabla \cdot(\mu \nabla \psi)=0
$$

where $\psi$ is the total magnetic potential. In the area of interface between total and reduced potentia : the limiting conditions of normal and tangential $\vec{B}$ being continuous (see Appendix $B$ ) are applied. Boundary conditions on the surfaces of the mesh are expressed as functions of the total potential $\psi$.

In TOSCA, the conductors are represented by finite element brick units as shown in Figure 17. This brick is defined by 20 nodes, each of which has 3 coordinates. Thus each brick is defined by 60 parameters. As can be seen, any conductor geometry can be fitted with this brick. Another component which has to be superimposed upon the geometry of the brick is the current density which crosses the surfaces of the brick. Complex conductor geometries, such as those used in force-free coils, require detailed description of each conductor brick. The geometry description and code formulation of the coil is often tedious and time consuming. The magnetic 


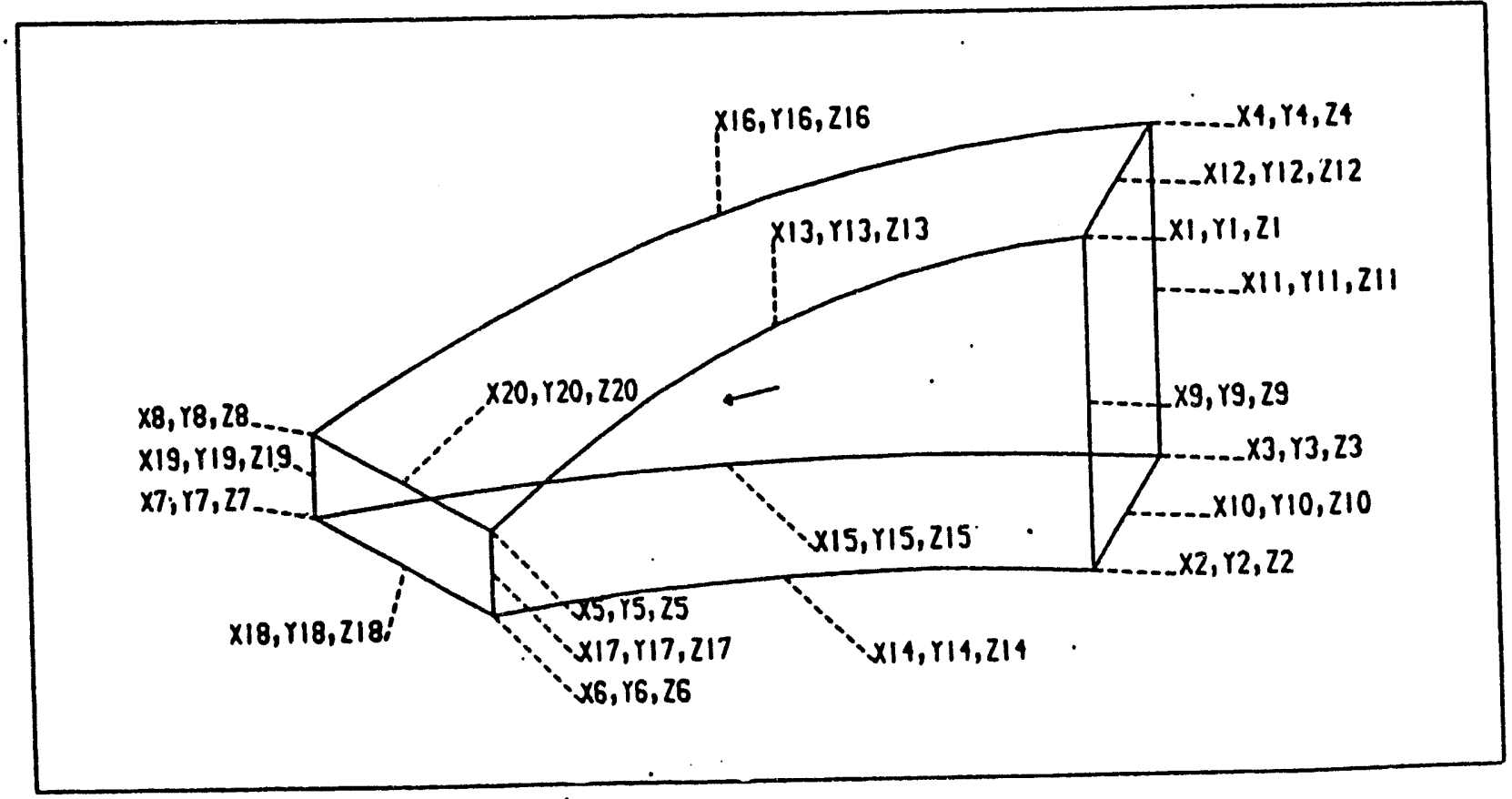

Figure 17. Typical finite element 20 node brick 
field is calculated over a three-dimensional computational mesh which covers the conductor mesh and regions surrounding the conductor. 


\section{Preliminary Comparative Study of Force-Reduced Magnets with Conventional Magnets}

Recent interests in MHD propulsion for marine vehicles have rece 'ied the U.S. and Japan to investigate large-scale MHD propulsion systems. One of the bottlenecks of the technology is the development of light-weight/high-field superconducting magnets. Several coil configurations have been proposed in the literature. In the following section, these configurations are first reviewed and a proposed force-reduced rectangular torsatron for MHD propulsion is analyzed and compared to a conventional racetrack configuration.

\section{4(a). Review of Proposed MHD Thruster Configurations}

NUSC (Naval Underwater Systems Center) has proposed to study several MHD thruster configurations. Some of these proposed configurations, shown in Figure 18 and 19, are a) the double solenoid, b) the clustered dipole, and c) the toroidal annulus. In the double solenoid geometry, two concentric ducts hold the electrodes with an inner and outer solenoid magnet. The current flows radially between the radial current and the axial magnetic field creates an azimuthal thrust that is redirected in the axial direction by means of helicoidally shaped vanes. The second configuration is the clustered dipole geometry where several cylindrical thrusting sections with superconducting saddle-shaped dipole magnets are clustered around a central cylindrical duct. This clustered configuration is the one used by the Japan Foundation for Shipbuilding Advancement (JAFSA). The third configuration is the toroidal annulus thruster configuration which is shown in Figure 19(a) and mounted on a submarine vehicle in Figure 19(b).

For both types of configuration (saddle dipole and toroidal annulus), the large operating magnetic field introduces a strong magnetic pressure on the central cylinder, as it is to the bucking post of a Tokamak. This central region is prone to much of the mechanical stress resulting from electromagnetic forces. To relax this pressure, one can devise a force reduced coil configuration as shown in Figure 20, whereby a helicoidal torsatron of two helices is mounted around the submarine. The water would flow between the coils and perpendicular to the plane of Figure 20(a). The resulting field in the torsatron is mainly toroidal. As discussed earlier, the torsatron is a force-reduced configuration provided that the helical pitch is appropriately chosen.

In the following section, a brief introduction on the torsatron is first given, and an attempt to compare electromagnetic forces for a rectangular torsatron and a racetrack configuration is then made. 
a

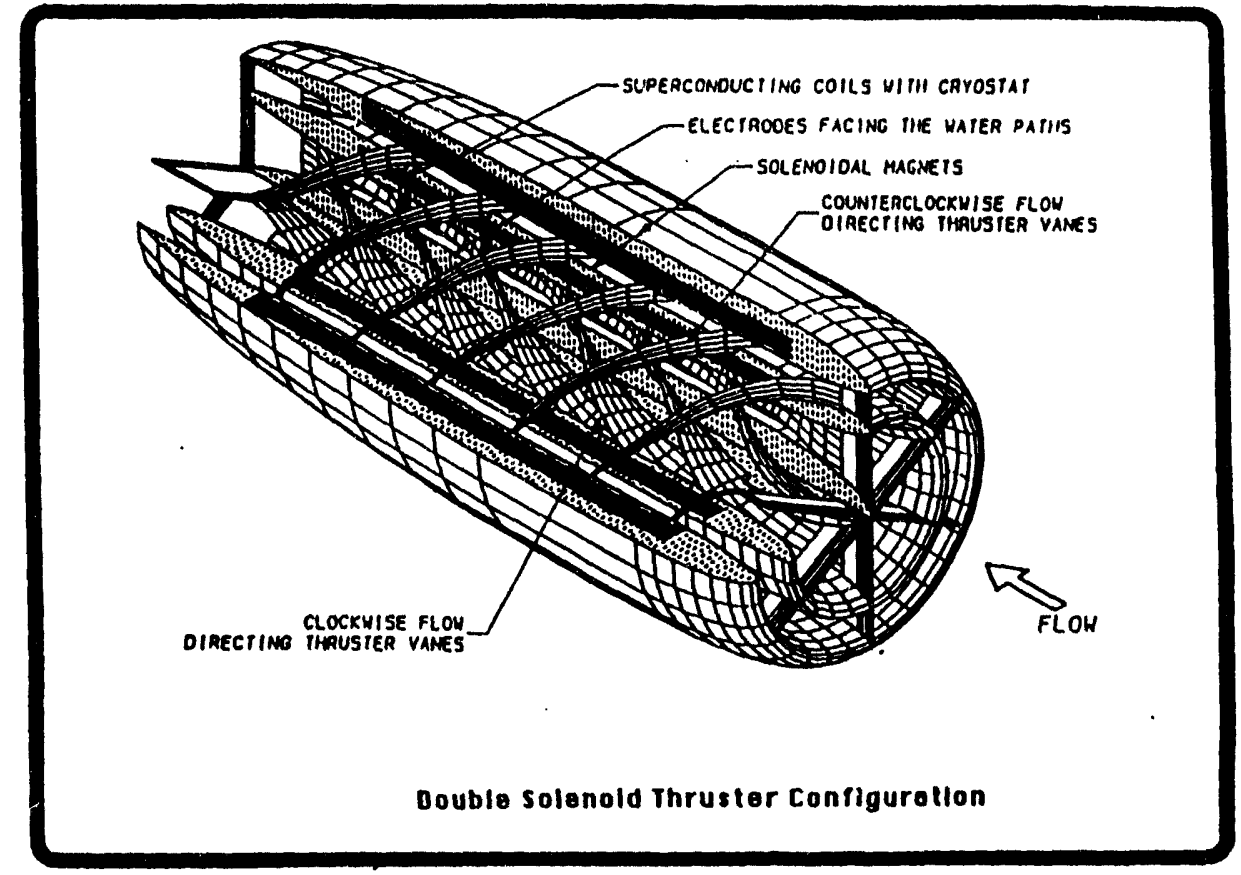

b

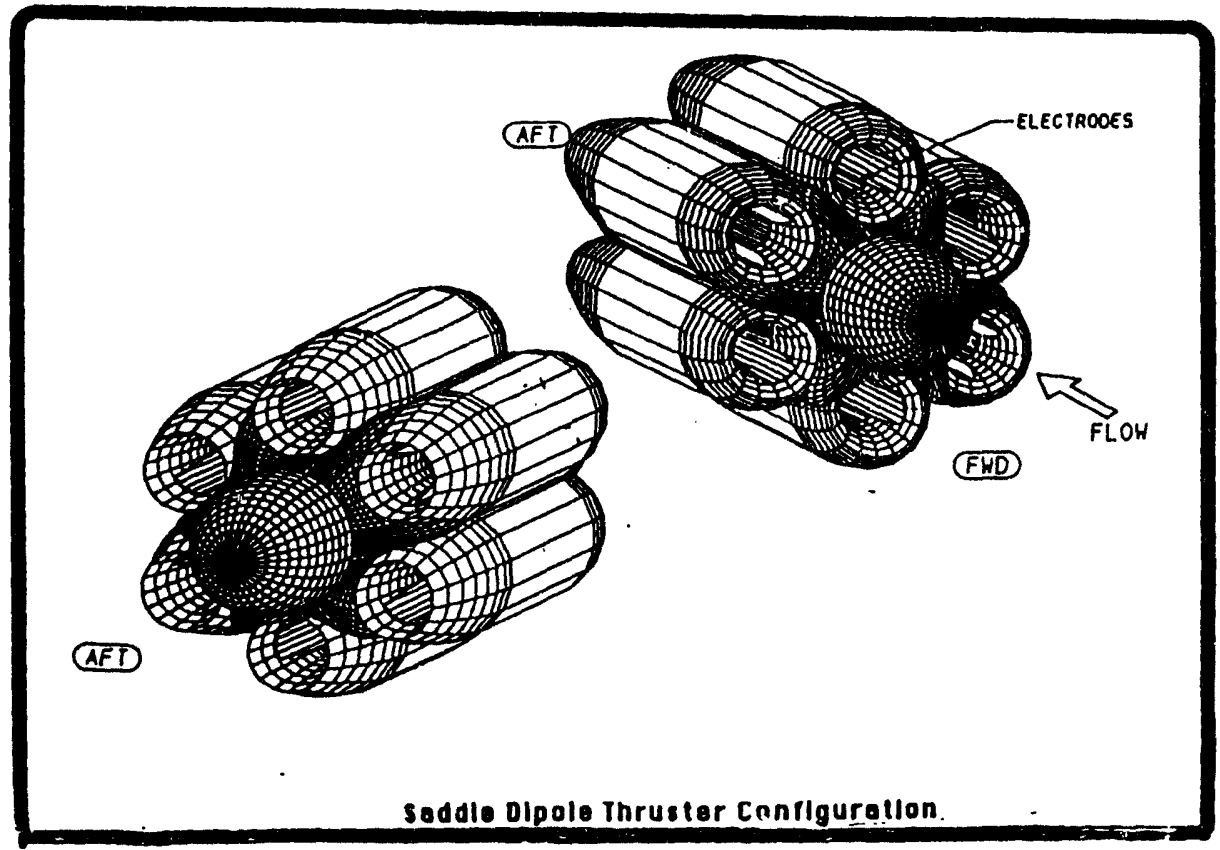

Figure 18. Two possible thruster configurations a) the double solenoid thruster and b) the saddle dipole thruster configurations 
a

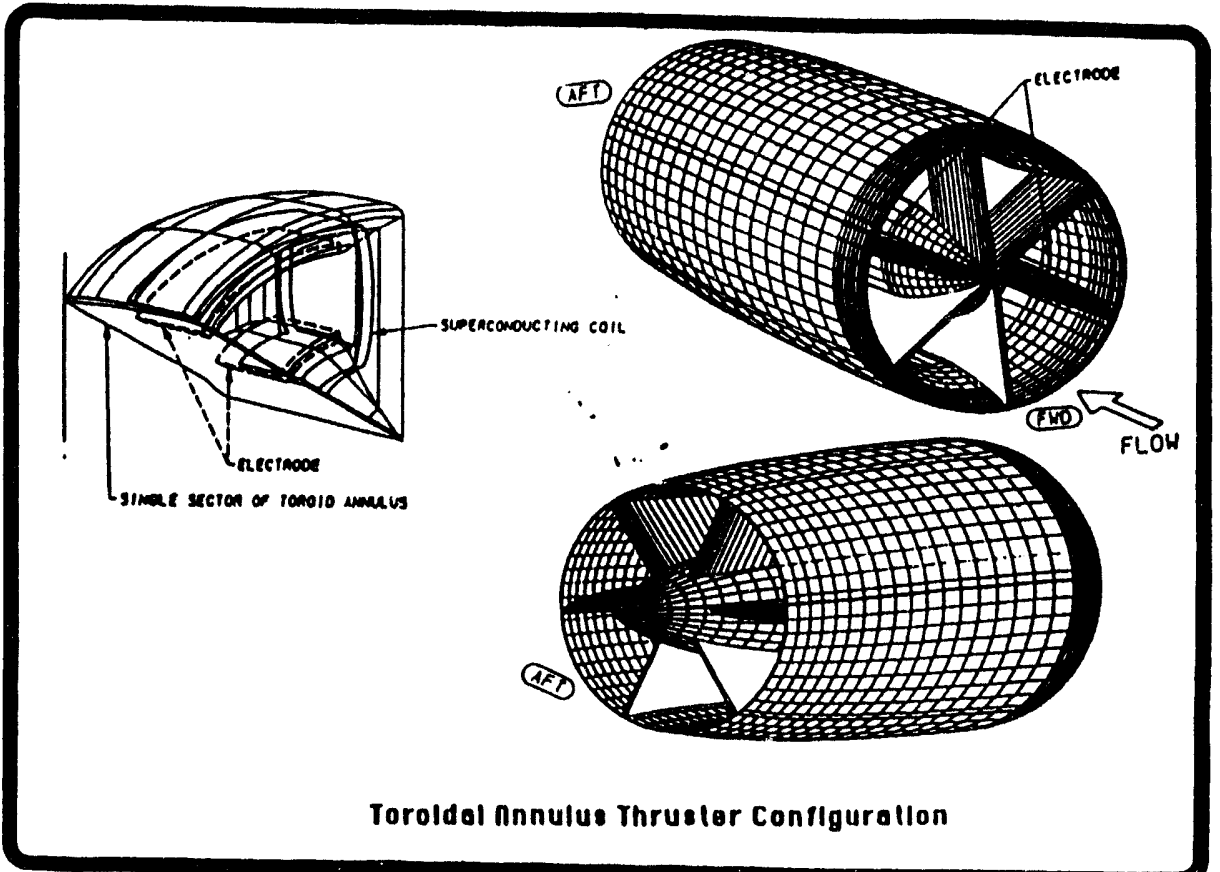

b

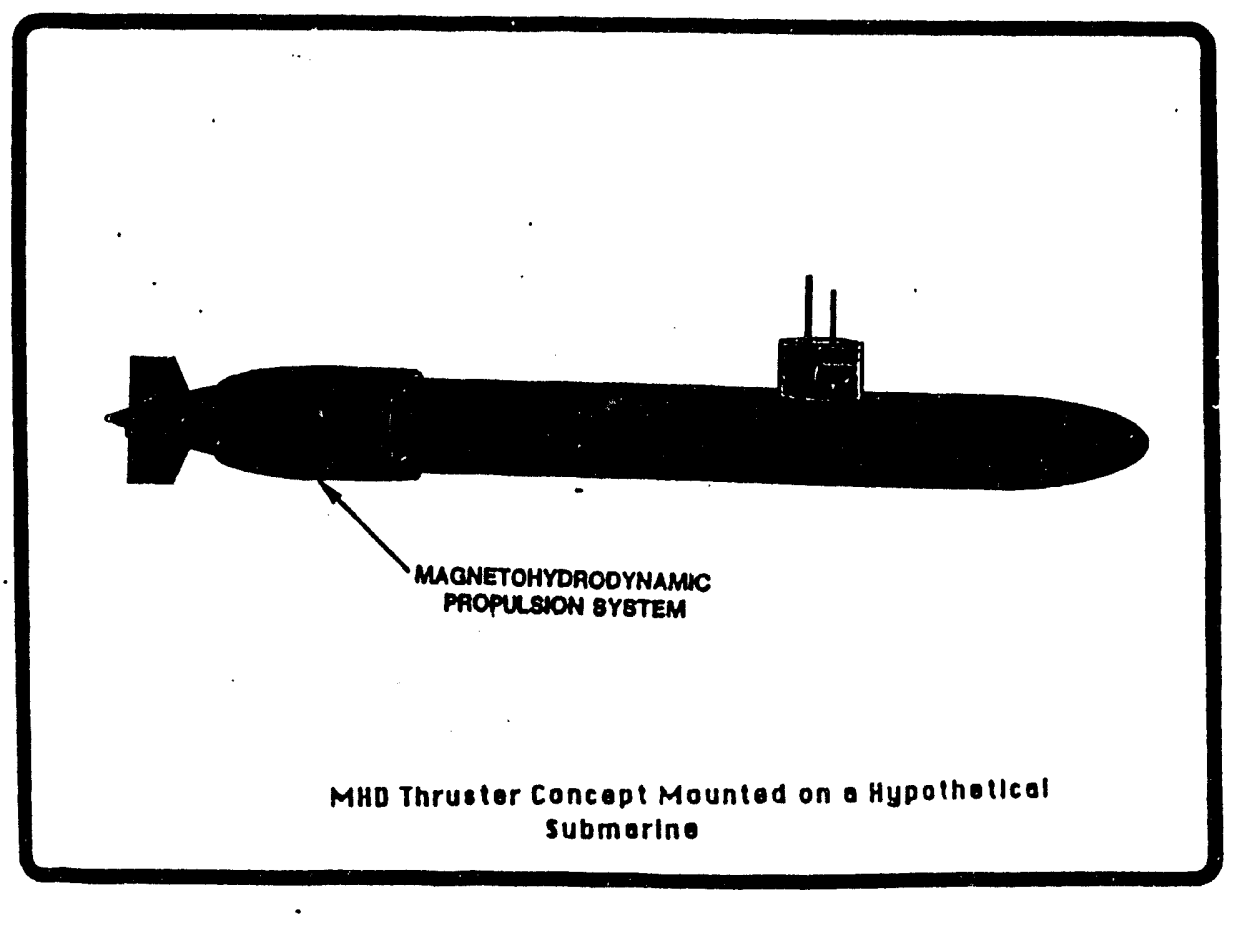

Figure 19. a) Toroidal annulus thruster configuration, b) Mounted on a submarine vehicle. 
Section A-A

$\mathbf{a}$

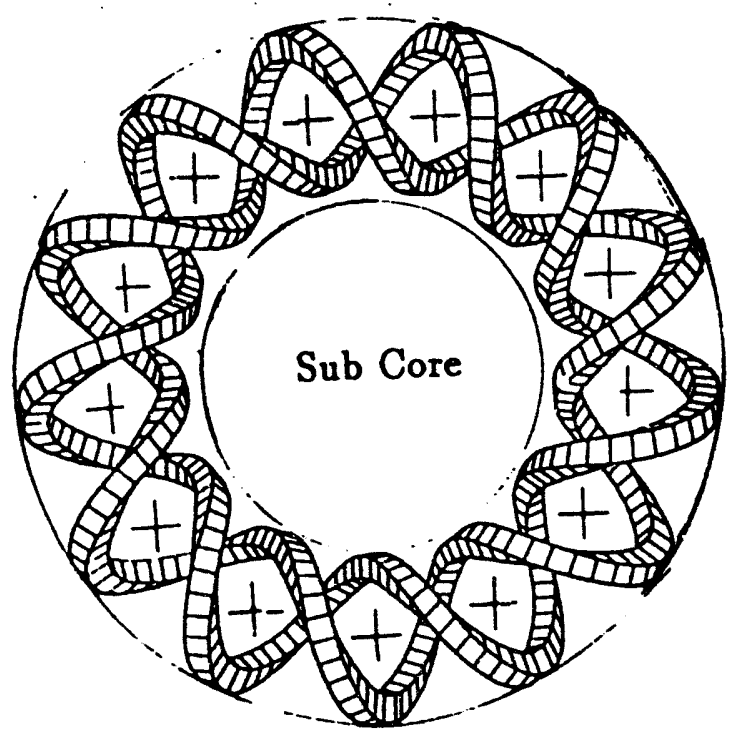

+ Seawater flow

b)

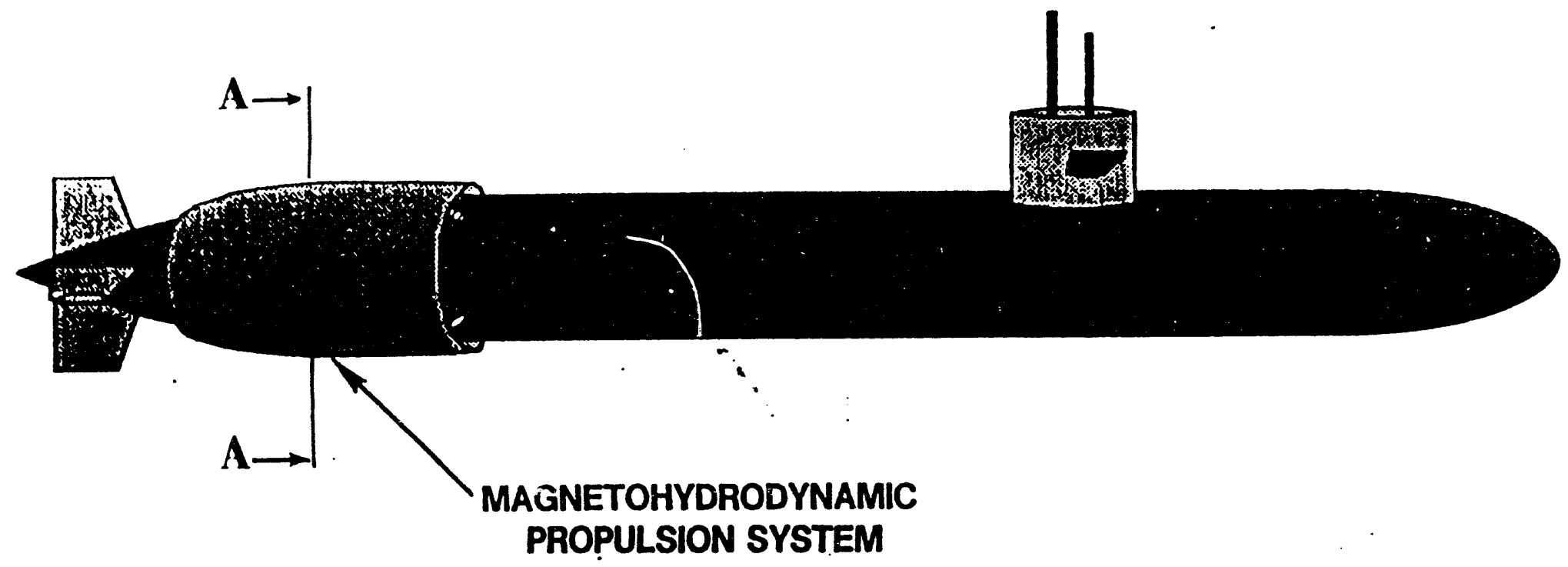

Figure 20. a) Cross section A.A. of a proposed torsatron thruster mounted on the submarine (not to scale), b) typical toroidal magnet mounted on the submarine shell 


\section{4(b). Torsatron Analysis}

A torsatron coil configuration is made of several helices. By properly adjusting each helix pitch, one can obtain a force-reduced coil system. Let us consider a helix which can be represented by the parametric coordinates (as shown in Figure 21)

$$
\begin{aligned}
& r=r \operatorname{Cos} \theta \\
& y=r \operatorname{Sin} \theta \\
& z=h \theta
\end{aligned}
$$

As readily seen, for an angle displacement $\theta$, the point on the helix moves by $h \theta$ in the vertical direction and rotates by the angle $\theta$ on the circle of radius $r$ in the horizontal plane. The Frenet trihedron which consists of a tangent, normal and binormal, is defined by the Frenet-Serret equations which read

$$
\begin{aligned}
& \frac{d T}{d s}=\kappa N \\
& \frac{d N}{d s}=-\kappa T+\tau B \\
& \frac{d B}{d s}=-\tau N .
\end{aligned}
$$

Where T, N and B are the tangent, the normal and the binormal vectors to the helix. $K$ and $\tau$ are called the curvature and the torsion, respectively. The tangent vector is given by $\left(-\sin \theta, \cos \theta, \frac{h}{r}\right)$ where $\operatorname{tang} \alpha=h / r$ and $\alpha$ is the helix angle. If another helical conductor has its tangent vector perpendicular to the first helix tangent, the perpendicularity constraint imposes that:

$$
\operatorname{Sin}\left(\theta+\frac{2 \pi}{n}\right) \cdot \operatorname{Sin} \theta+\operatorname{Cos} \theta \cdot \operatorname{Cos}\left(\theta+\frac{2 \pi}{n}\right)+(h / r)^{2}=0
$$

which is equivalent to

$$
(h / r)^{2}=-\operatorname{Cos} \frac{2 \pi}{n}
$$

if $n=2$; which is a system of two helically torsated coils; we find that $h / r=1$ or $\alpha=45^{\circ}$. If $n=3$, which is a system of three helically torsated wires, we find that $\alpha=35^{\circ}(\mathrm{h} / \mathrm{r}=1 / \sqrt{2})$. Hence, in a "force-free" torsatron configuration, the three helices depart from the horizontal plane with a 


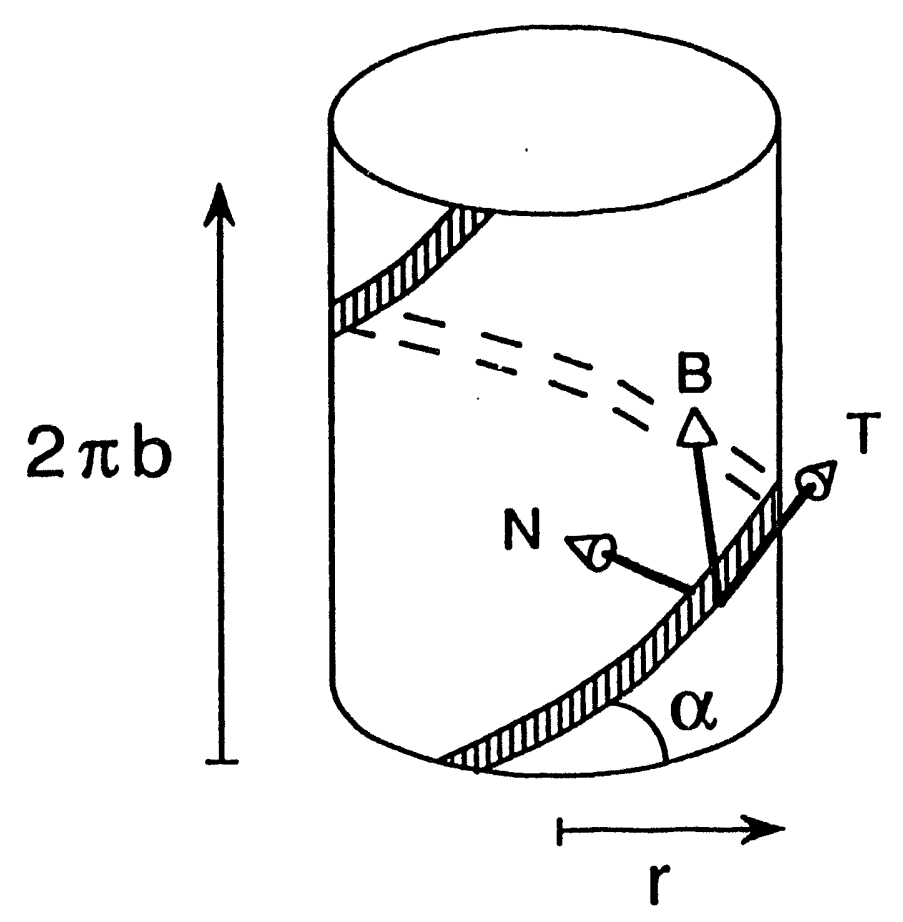

Figure 21. Geometry of a helix 
tangent angle of $35^{\circ}$. Other configurations are possible for $n>3$ provided that $\operatorname{Cos}\left(\frac{2 \pi}{n}\right)$ is negative. The system of two helically torsated coils was shown in Figure 20(a) and a system of three helically torsated coils was shown in Figure 11.

\section{4(c). Preliminary Comparison of Electromagnetic Forces Between Torsatron and Conventional Systems}

To understand why and how forces are reduced in a helical winding system, we used the TOSCA computer code to compute the magnetic field generated by a helix and the corresponding 4-ring systems as shown in Figures 22 and 23. The helix angle is $45^{\circ}$ and the conductor is a 0.01 $\mathrm{cm}$ diameter cylindrical wire. The radius of the helix and of the circular rings are $5 \mathrm{~cm}$. The current density passing through the helical coil is $10 \mathrm{~A} / \mathrm{cm}^{2}$ and that of the rings has been adjusted to maintain a constant total ampere-meter number for both systems. Such argument was used by Moses (1975) in his comparison study of superconducting magnet scaling laws. In brief, he argued that the critical current density is fixed to a certain degree by the conductor design. Hence, fixing a number of amperes would then fix the cross section of the conductor. By fixing the total ampere meters for the coils, the volume of the conductor becomes fixed. Moses further argues that the quantity of stabilizer is proportional to the ampere-meter number and so are the helium inventory and assembly mass and costs.

The magnetic field intensity histograms for the two configurations are shown in Figures 24 and 25 and Figures 26 and 27, respectively. As can be seen, the field intensity is locally larger inside the rings for the 4-ring system than that for the helical system, though the 4-ring system is sustaining a spatially averaged field density $30 \%$ smaller. The Lorentz force exerted on the 4-ring system was computed to be about $22 \%$ greater than that exerted on the helix, for the same conductor element size basis.

This example brings about several important characteristics of these two systems. First, the field generated by a single helical coil spreads more in space, as shown in Figures 24 and 25. This tends to produce a smaller field gradients on the coils and thus to impose smaller electromagnetic forces on the coils. In contrast, in a ring the field is "compressed" which tends to create more force on the winding because of larger field gradients. This example shows that minimum forces are created on the coils when the magnetic pressure gradients are minimum. In the helical coil, the magnetic field spreads more in space, yielding smaller gradients in the neighborhood of the coils, and thus smaller forces on the coils. The strength of these fields and field gradients for these two systems are represented by a shading gray palette in Figures 24 and 25 and histogram on Figures 25 and 26. 


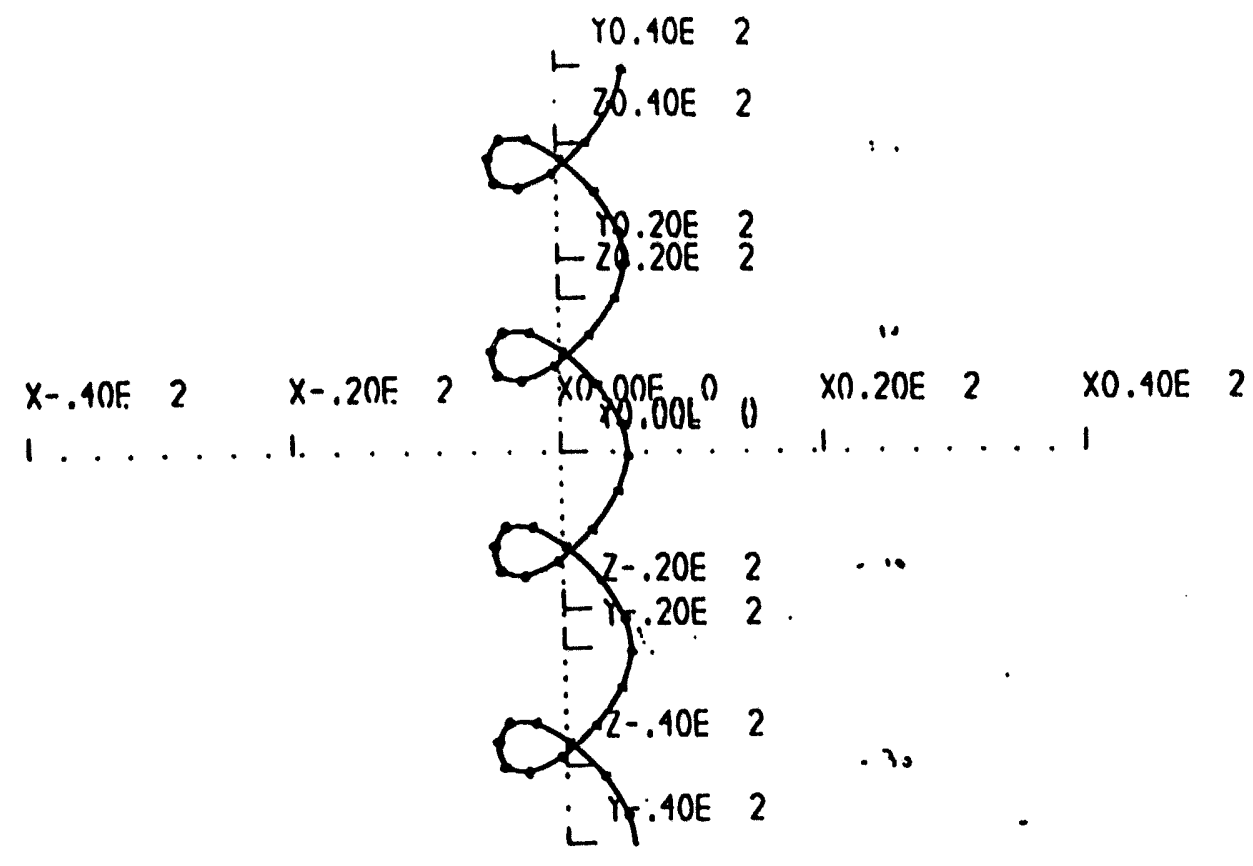

Figure 22. Single helix torsatron 


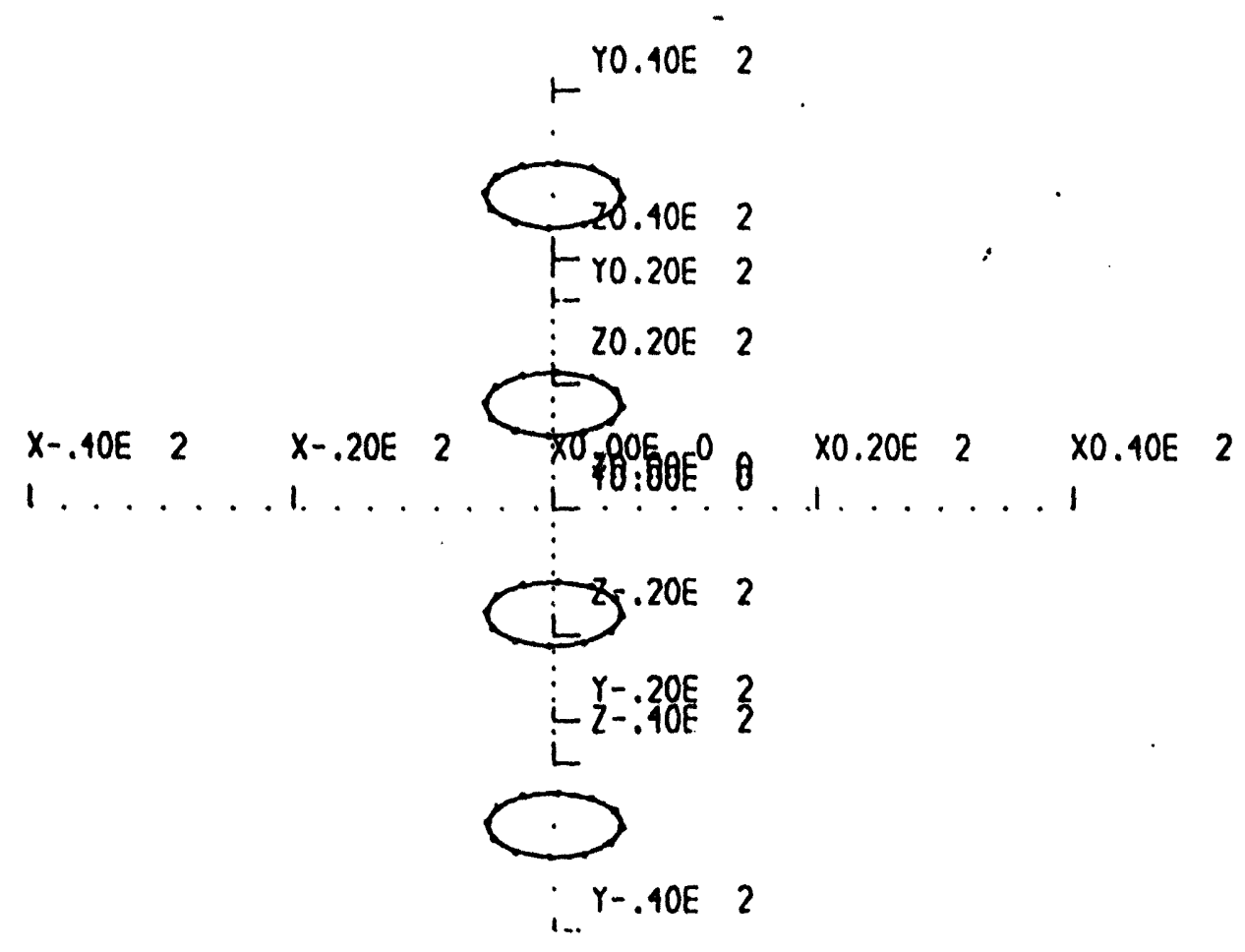

Figure 23 Four ring systems 
Figure 24. Magnetic field intensity distribution for a single wire torsatron 


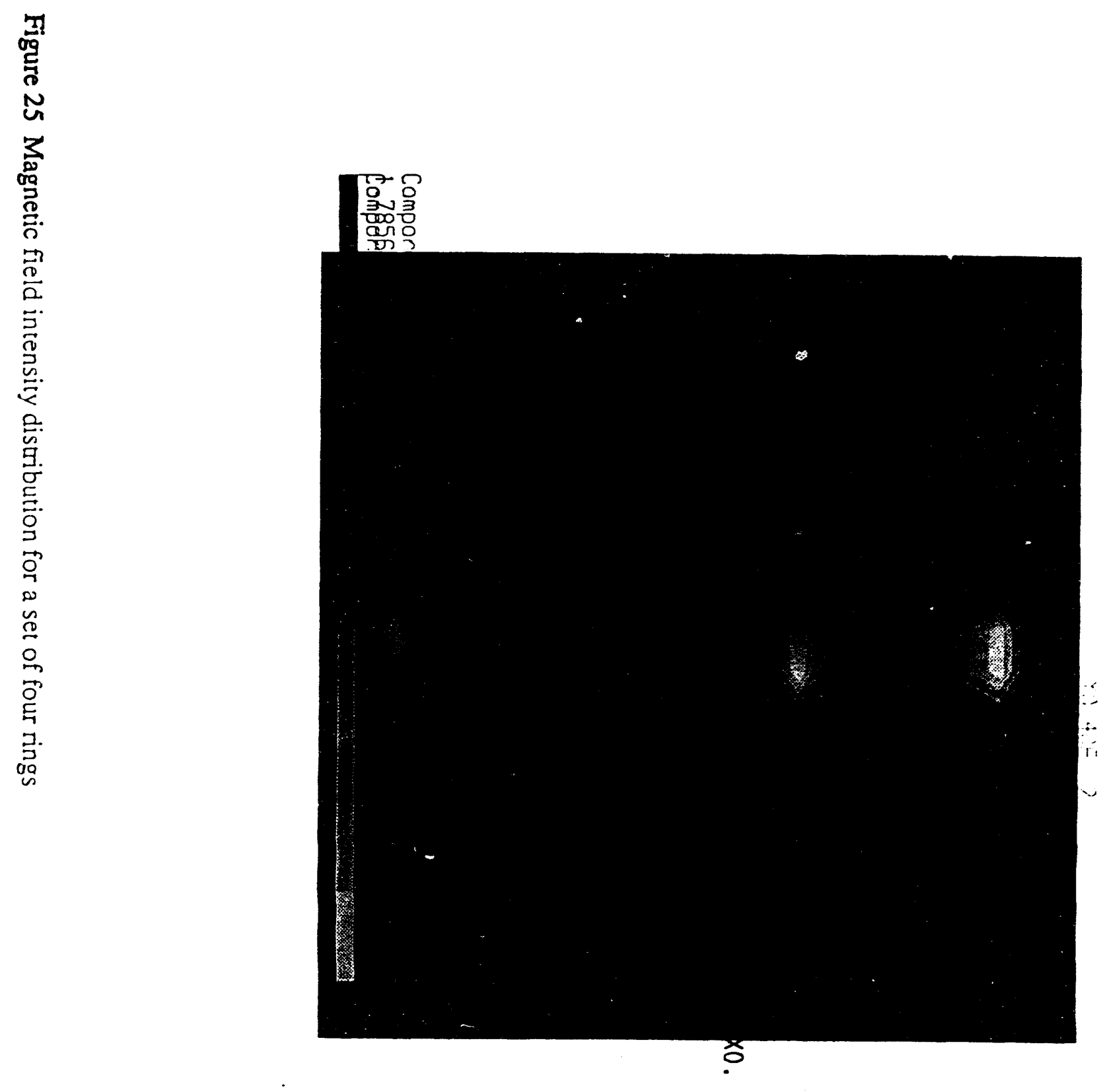




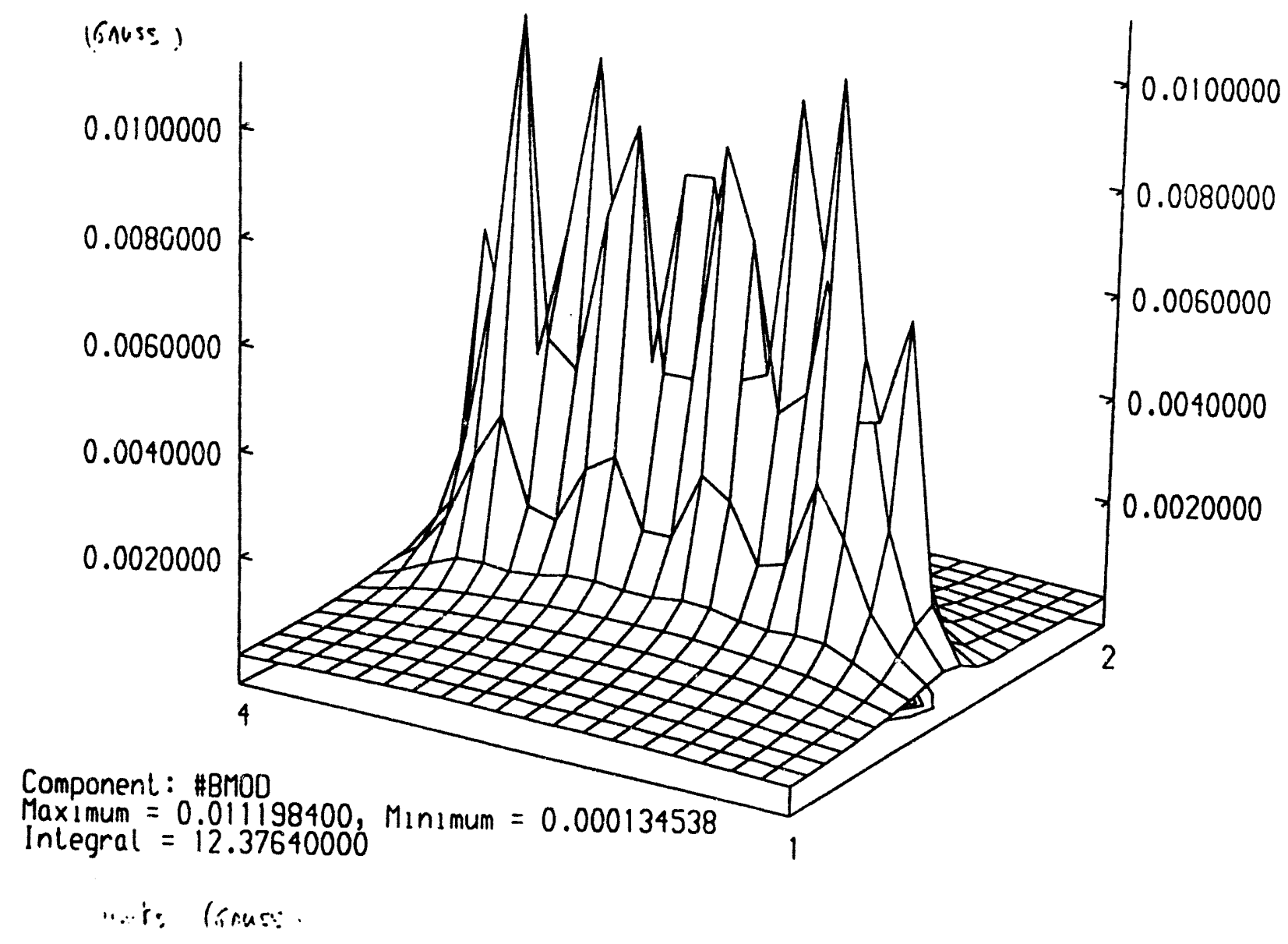

Figure 26. Histogram of the magnetic field intensity for the single wire torsatron 


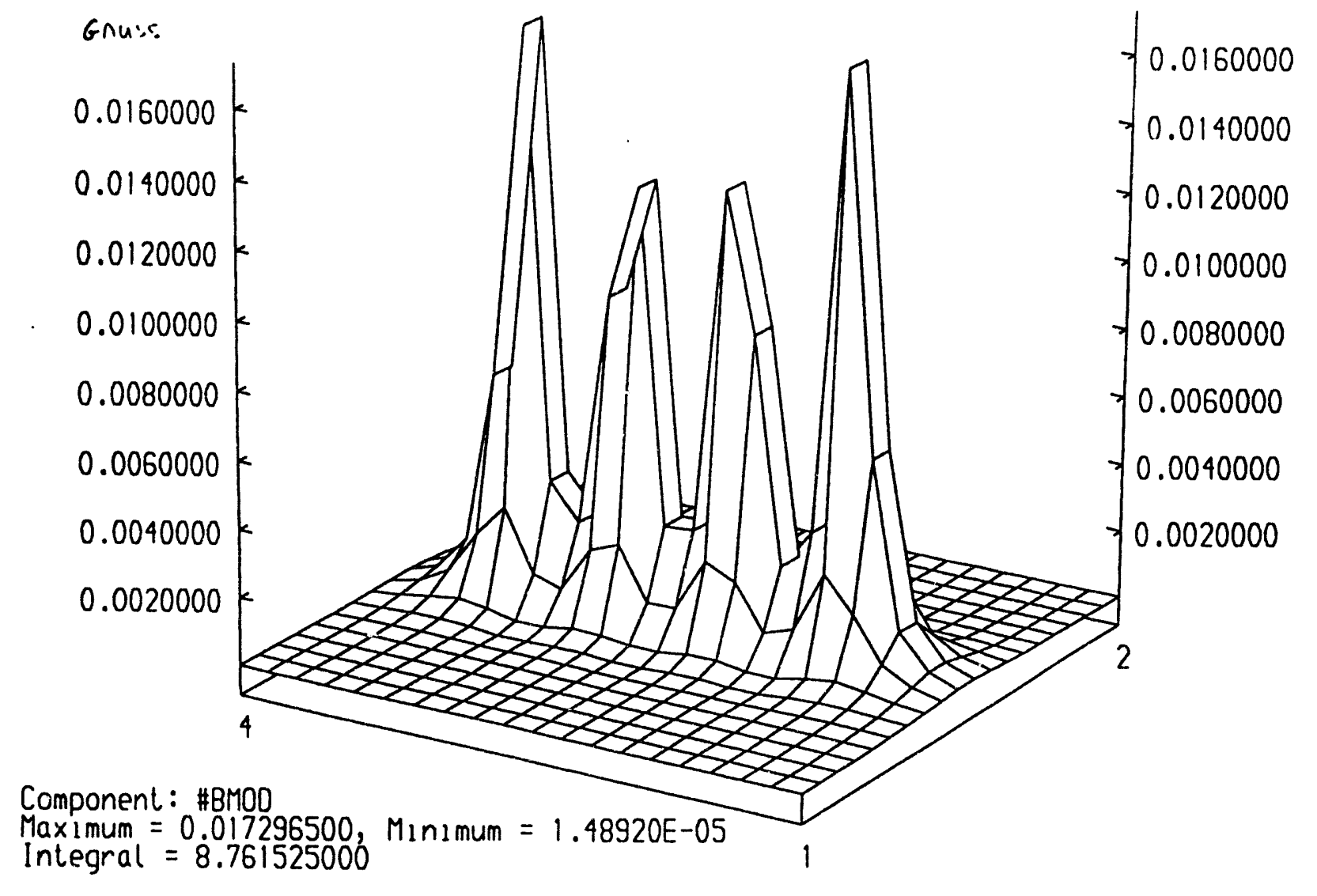

Figure 27. Histogram of the magnetic field intensity for the four ring set 
Another coil geometry relevant to MHD ship propulsion was investigated. It is a two-helix rectangular torsatron which is illustrated in Figure 28. In this configuration only two wires are used and are crossing each other at $90^{\circ}$. To simplify the analysis of this system only a portion of this winding is analyzed as shown in Figure 29. At the boundary of the computational domain, symmetry is assumed. An equivalent racetrack coil is shown in Figure 30, which has the same radial and lateral dimensions. To compare these two systems on the same basis, the current density in the equivalent racetrack coil is adjusted so that the total amperemeters are the same for both configurations following the arguments discussed above. The geometrical ratios are such that $\mathrm{r} / \mathrm{L}=1$ and $\Delta \mathrm{r} / \mathrm{r}=0.01$ (see Figure 28). These aspect ratios were suggested as typical for MHD toroidal thruster mounted on a submarine vehicle. Schematics for these two coils configurations are indicated in Figures 29 and 30. The computed forces exerted by the regular torsatron on the radial portions of the torsatron are about 2.7 times smaller than that exerted on the radial portions of the racetrack. A force reduction of about 20 times is calculated on the lateral portions of the torsatron coil with respect to the lateral portion of the racetrack.

This example illustrates that spreading the field over the coil as it is the case in a rectangular torsatron, yields a significant force reduction on the wires. Using such configurations, one could therefore hope to generate higher fields with less structural constraints .

Although forces are being reduced in such configurations, one may realize that the resulting magnetic field is tortuous and highly nonuniform. This could lead to nonuniform momentum thrust that could induce unnecessary turbulence and possible flow reversal, thus seriously limiting the hydrodynamic and acoustic performances of the thruster.

\section{4(d). Comparative Study of Toroidal Pod and Toroidal Annulus Conventional Configurations for MHD Propulsion}

To gain further insight and provide a basis for magnet performance comparison, two conventional geometries were studied and are presented in Figures 31 and 32 for a six racetrack toroidal pod and a 8 racetrack toroidal annulus configurations. A same current density $4000 \mathrm{~A} / \mathrm{cm}^{2}$ is assumed in both configurations, which is an upper bound for the current density presently used in superconducting magnets. As a reminder, the 6 Tesla ANL superconductor (Nb (48\%) Ti) dipole magnet was designed for a current density of about $2000 \mathrm{~A} / \mathrm{cm}^{2}$. As can be seen in Figure 33 , this current is well within the envelope of the conductor characteristics. 


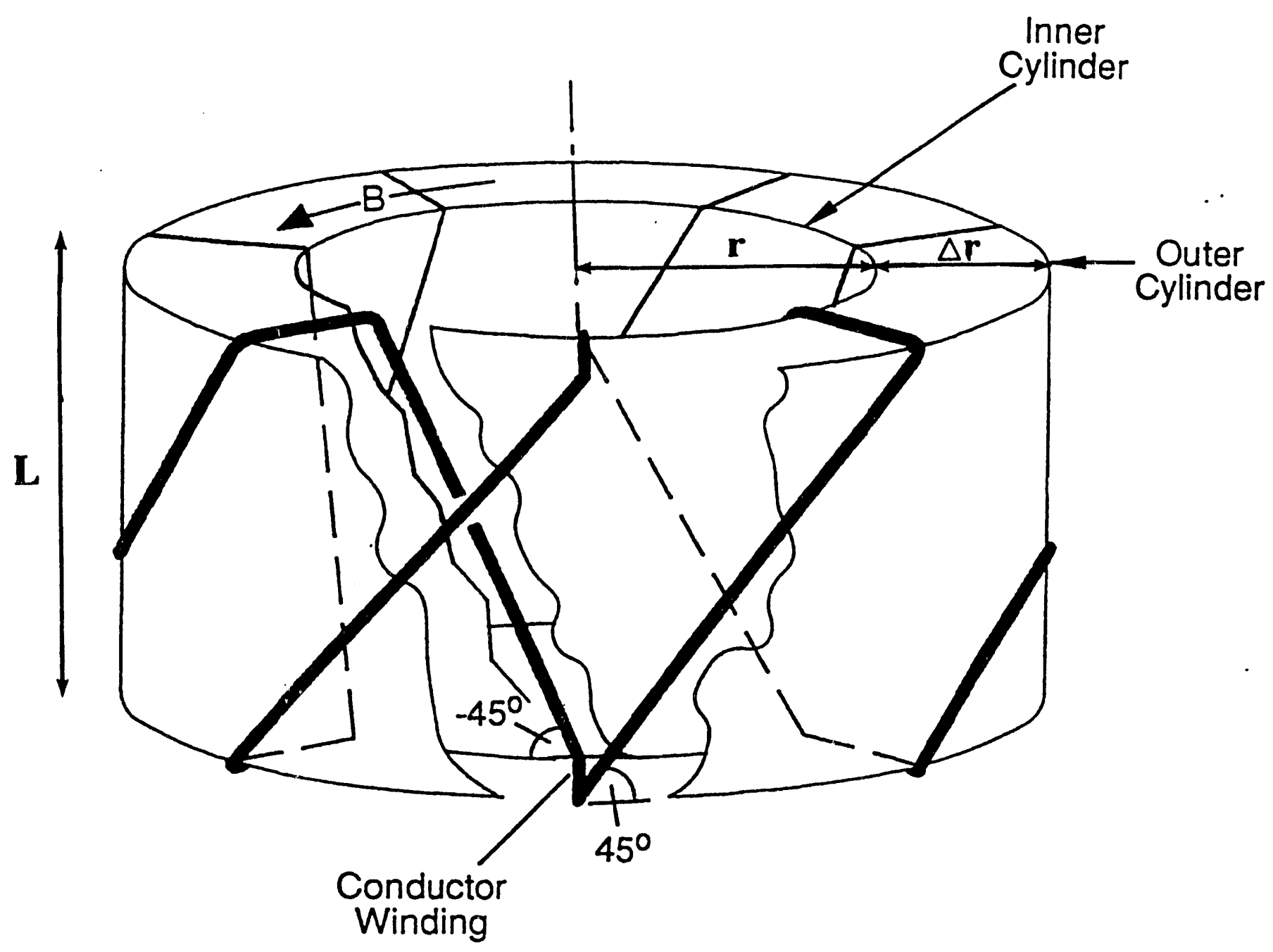

Figure 28. Coil Winding Configuration for two-helix rectangular toroidal magnet torsatron concept 


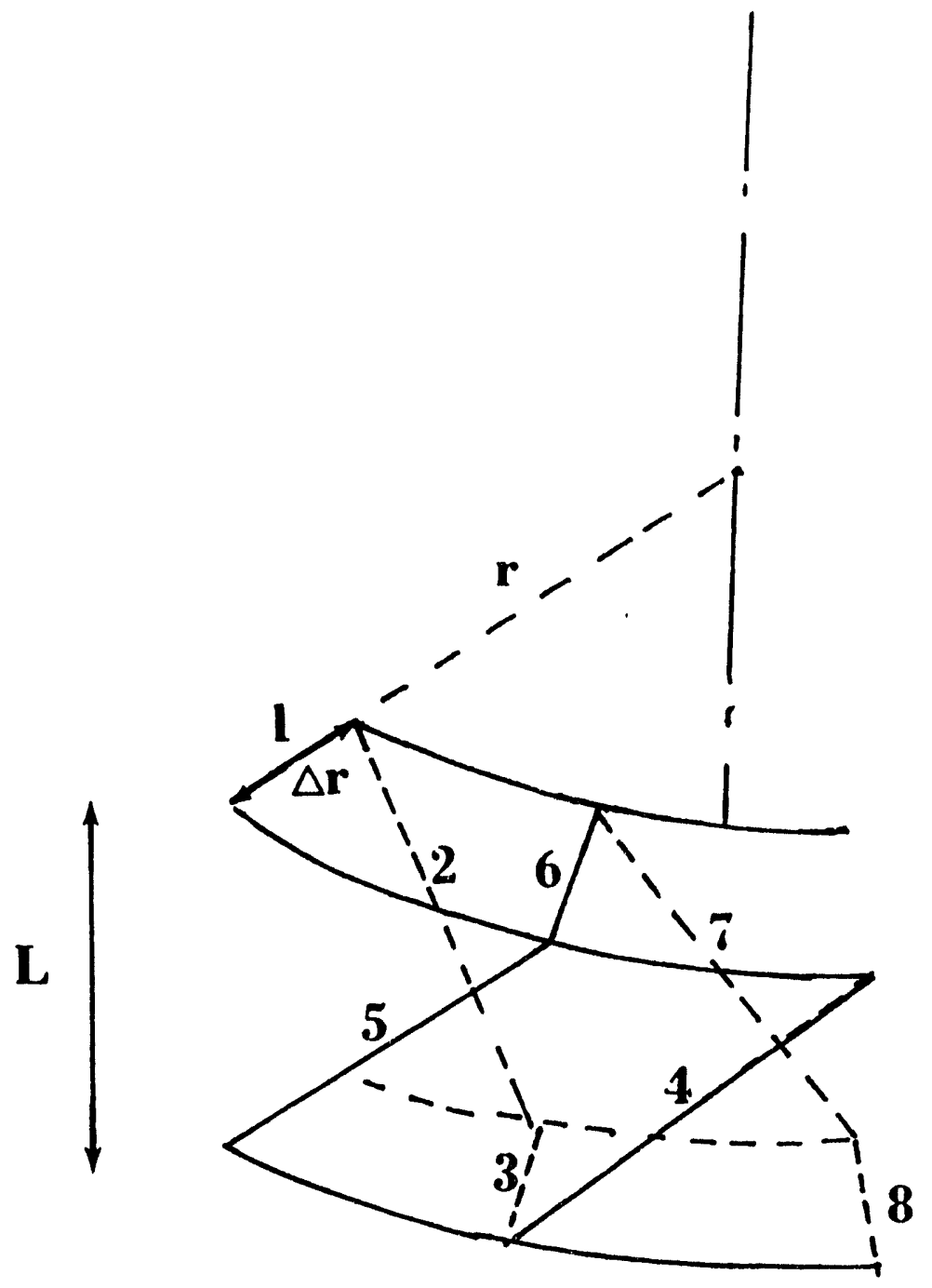

Figure 29 Two-helix rectangular torsatron 


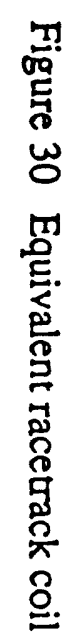

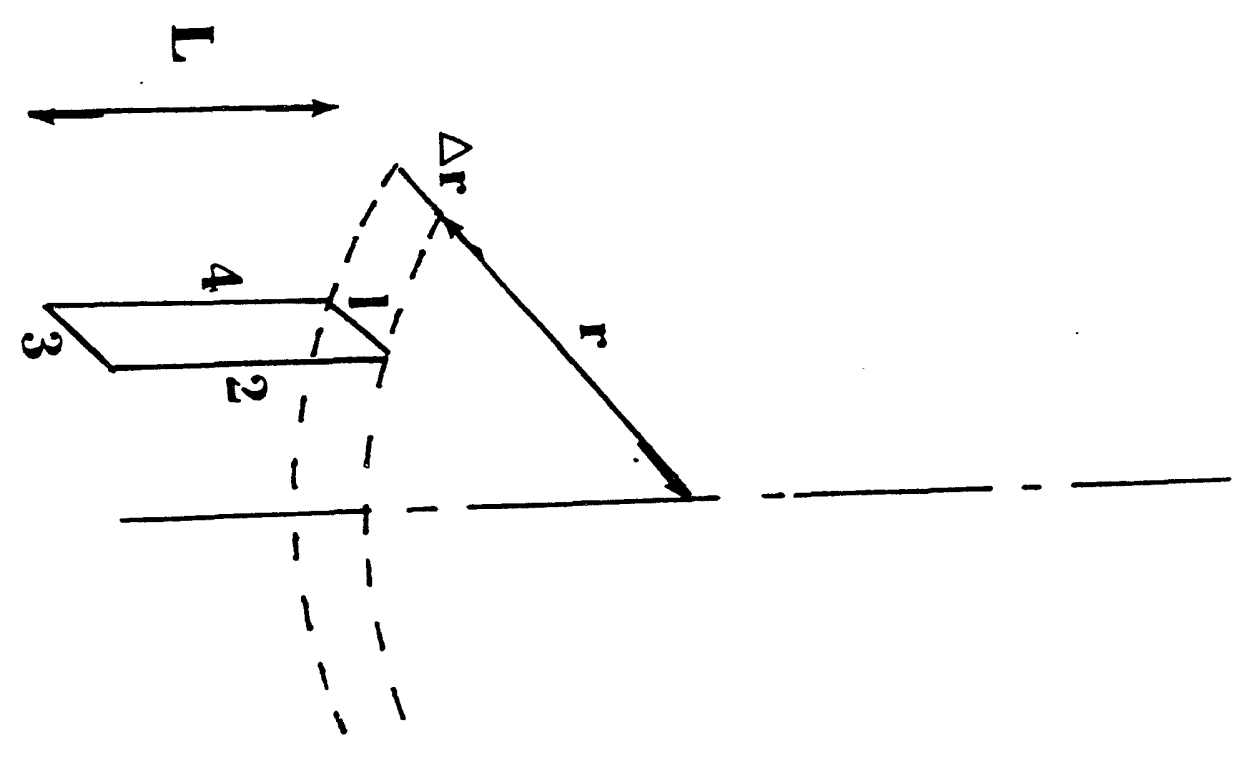




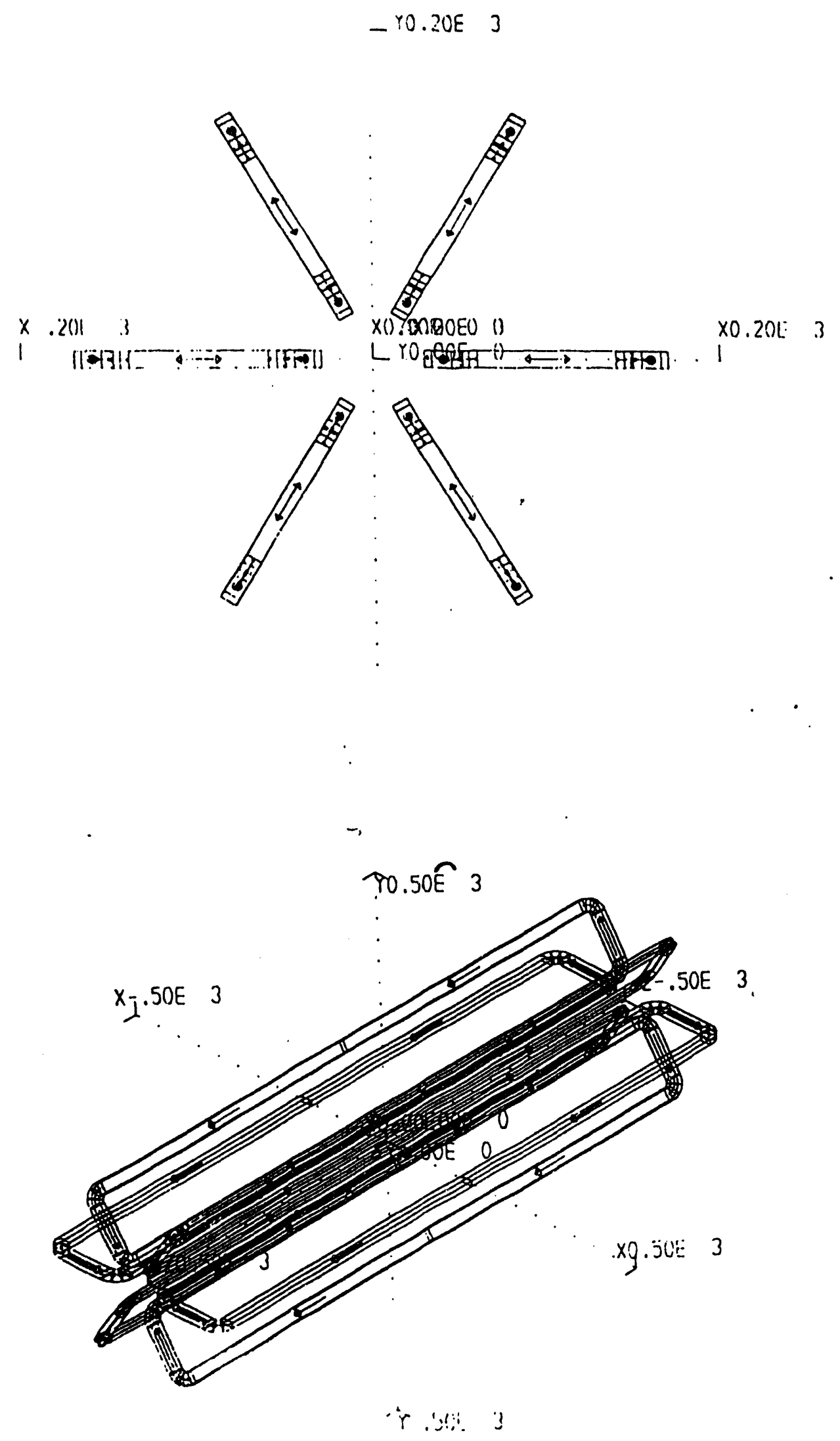

Figure 31 Six racetrack configuration 


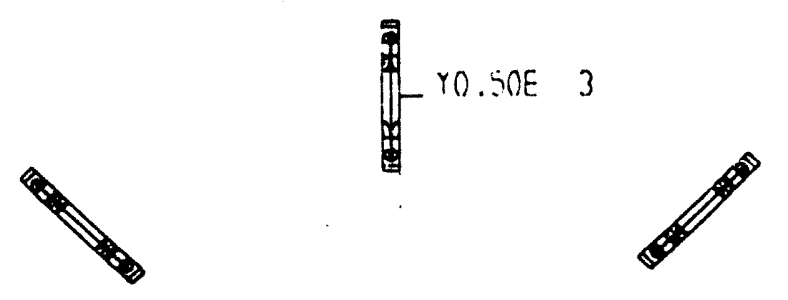

$\begin{array}{lll}X-.50 E \quad 3 & \text { XOZOOEOEO } 0 & \times 0.50 E \\ L Y O .00 E .0 & 0\end{array}$

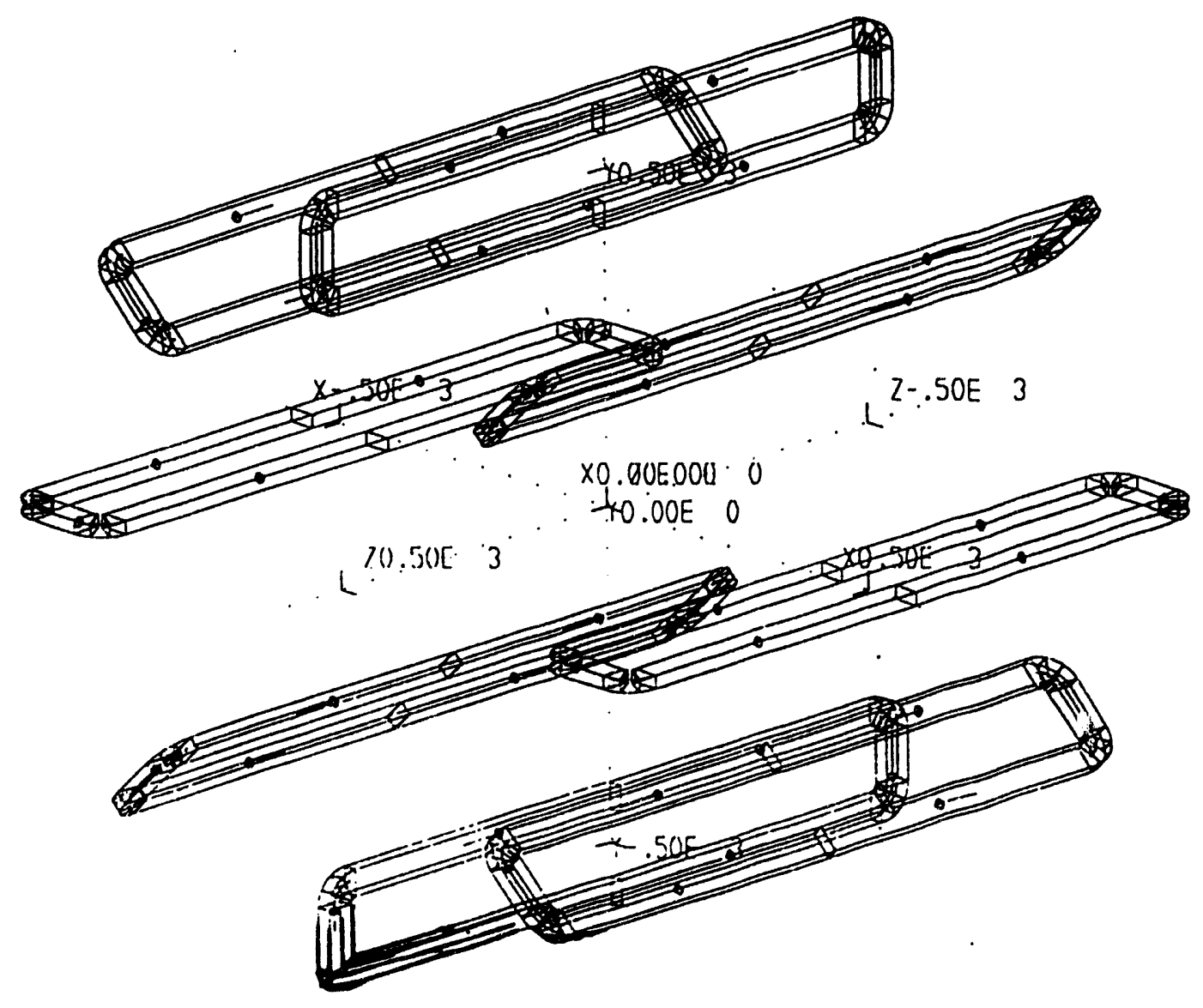

Figure 32 Eight racetrack configuration 
The first configuration consists of 6 racetracks as shown in Figure 31. Each individual racetrack length is about $10 \mathrm{~m}$. Two diametrically opposed racetracks are separated by about $1 \mathrm{~m}$. The radial size of each racetrack is about $1 \mathrm{~m}$. Each racetrack winding supports a conductor crosssection of $20 \mathrm{~cm}$ radially by $10 \mathrm{~cm}$ azimuthally. The total current flowing in each racetrack is 4000 $\times 20 \times 10=810^{5}$ Amps turn. In Figure 34(a), a plot showing the magnitude of the magnetic field is presented. The magnetic field was evaluated at mid-axial length of the racetrack. The peak field occurs in the inner leg portion of the racetrack coil at a value of 2.7 Teslas. The field inside the racetrack volume drops to a value of 1.0 Tesla. The peak field in the outer leg of the racetrack is about 2.2 Teslas. As can be seen in Figure 34(a), the field is highly nonuniform in the toroidal propulsing volume between the racetracks. This design for an MHD thruster would likely produce nonuniform thrust, with a thrust maximal in the regions close to the inner radius core of the magnet. Figure 39(b) shows a similar plot from another point of view. This figure illustrates better the rapid change of the field near the center core of the magnet configuration. Figure 34(b) also shows a relatively low field in the central portion of the magnet. The resulting large field gradients are causing a strong implosive force on the inner legs of the racetracks.

The other eight racetrack configuration, shown in Figure 32, has racetracks of the same dimensions as those used in the six coil configurations discussed above. They are equally spaced over a larger internal diameter of $10 \mathrm{~m}$. For the same current density $\left(4000 \mathrm{~A} / \mathrm{cm}^{2}\right)$, the peak value of the field drops to 1.9 Tesla, while the inner toroidal value of the field in a racetrack cross-section is reduced to 0.7 Tesla (Figure 35). The field drop-off between the racetracks is less pronounced than that for the 6 racetrack configurations because it follows $1 / r$ variations with $r$, the radius being larger. The magnitude of the field is likewise much smaller. In the eight racetrack configuration, the working field is only about $0.4 \mathrm{~T}$ between two racetracks. As can be seen, the field between two racetracks is also nonuniform, but to a lesser extent than that for the 6 racetrack pod. The ratio of peak field to inner-racetrack field is also about 3 .

These two examples show that such proposed configurations are far from being ideal for MHD propulsion and need improvement. To further gain insight on these systems, a force calculation on each racetrack was performed. A typical racetrack is discretized in 18 elements as shown in Figure 36, with each individual element numbered from 1 to 18. Elements 9 and 10 constitute the inner legs of the interior central portion of the magnet, while elements 1 and 18 constitute the outer legs. The forces in $x, y$, and $z$ directions for both the six and eight racetrack configurations are shown in Tables 2 and 3, respectively. For both magnet configurations, the greater forces are exerted on the inner leg elements 9 and 10. For the six racetrack configuration, an inward force of about $4 \times 10^{6} \mathrm{~N}$ over a surface of $50 \times 10 \mathrm{~cm}^{2}$ results in a pressure of $80 \mathrm{MPa}$ 
acting on the inner legs. Note that the magnetic pressure inside the windings is only of the order of $\mathrm{B} 2 / 2 \mu \mathrm{o}$ which only translates into a pressure of $3 \mathrm{MPa}$. A quick evaluation of this term can be made by consulting the chart of Figure 42, where the magnetic field strength is directly converted into pressure. Typical superconducting windings can easily sustain a pressure of up to $180 \mathrm{MPa}$ ( $\mathrm{NbTi}$ ) or $300 \mathrm{MPa}\left(\mathrm{Nb}_{3} \mathrm{Sn}\right.$ ). In our example, the structural pressure exerted on the central bucking post is well below the commonly used structural material yield stress limit ( $300 \mathrm{MPa}$ ). Stronger materials can sustain even larger stresses of up to $1000 \mathrm{MPa}$.

For fields of the order of $10-20$ teslas, the superconductor $\mathrm{Nb}_{3} \mathrm{Sn}$ seems to be most appropriate. At these fields, the magnetic pressure in these superconductors is of the order of 10 $\mathrm{MPa}$, which translates into a strain of $0.01 \%$. (Assuming a commonly used Young Modulus of about $10^{5} \mathrm{MPa}$ for the conductor). The $\mathrm{Nb}_{3} \mathrm{Sn}$ superconductor belongs to the chemical composite category and has a strain sensitivity which is illustrated in Figure A-4. For a $20 \mathrm{~T}$ magnet, hoop stresses may reach about $20 \times 2 \times 10^{7}=400 \mathrm{MPa}$ (current density of $2 \times 10^{3} \mathrm{~A} / \mathrm{cm}^{2}$ and $1 \mathrm{~m}$ length coil radius assumed). This limit comes very close to the limit offered by the strongest structural superconductor materials available. In force-free configurations, these stresses should in principle become minimal, thereby opening the possibility of high field low specific weight magnets.

As explained earlier, true force-free mathematical solutions have often been established for infinite domains. For fininite coil geometry, however, these fields are no longer force free. It is, however, important to quantify how much force reduction could one expect when a force-free solution is truncated to a finite geometry. A numerical approach to this problem is discussed in the following section, for a Lundquist coil.

\section{4(e). Preliminary Estimate of Force-Reduction Using a Truncated Force- Eree Field Geometry}

In practice, force-free mathematical solutions over infinite domains may loose their forcefree characteristics if they are implemented for finite volume magnets. If the force-free solution is discretized and truncated, the solution is no longer "force-free" but may still be "force-reduced". It is the objective of this section to investigate the possibility of such force reductions. 


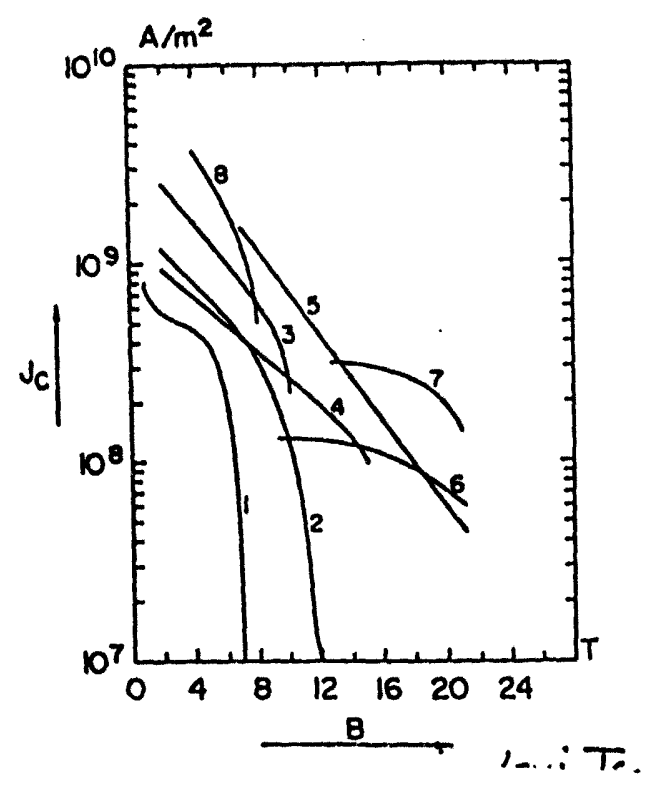

Figure 33 Short sample performance of cor ,non low Tc superconductors at 4.2K.

(1) $\mathrm{Nb}(25 \%) \mathrm{Zr}$, (2) $\mathrm{Nb}$ (48) Ti, (3) Nb(50Ti, (4) $\mathrm{Nb}_{3} \mathrm{Sn}$ (Vapor deposition, (5) $\mathrm{Nb}_{3} \mathrm{Sn}$ (Diffusion process), (6) $\mathrm{V}_{3} \mathrm{Ga}$ (Wire), (7) $\mathrm{V}_{3} \mathrm{Ga}$ (Ribbon),

(8) $\mathrm{Nb}(\mathrm{x}) \mathrm{Ti}$ 

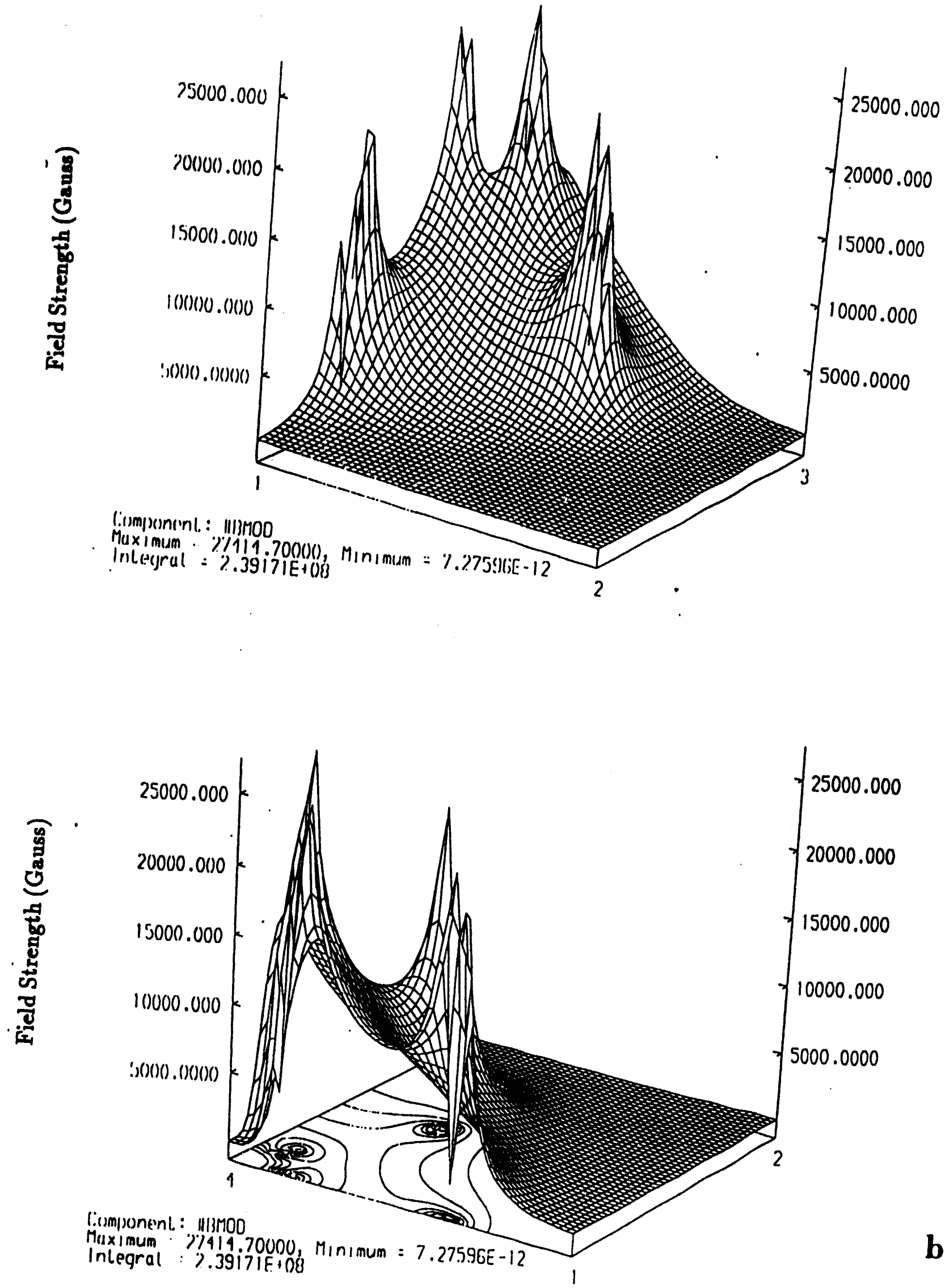

Figure 34 Computed magnetic field magnitude map at mid-axial length covering two racetracks of
the six racetrack magnet 


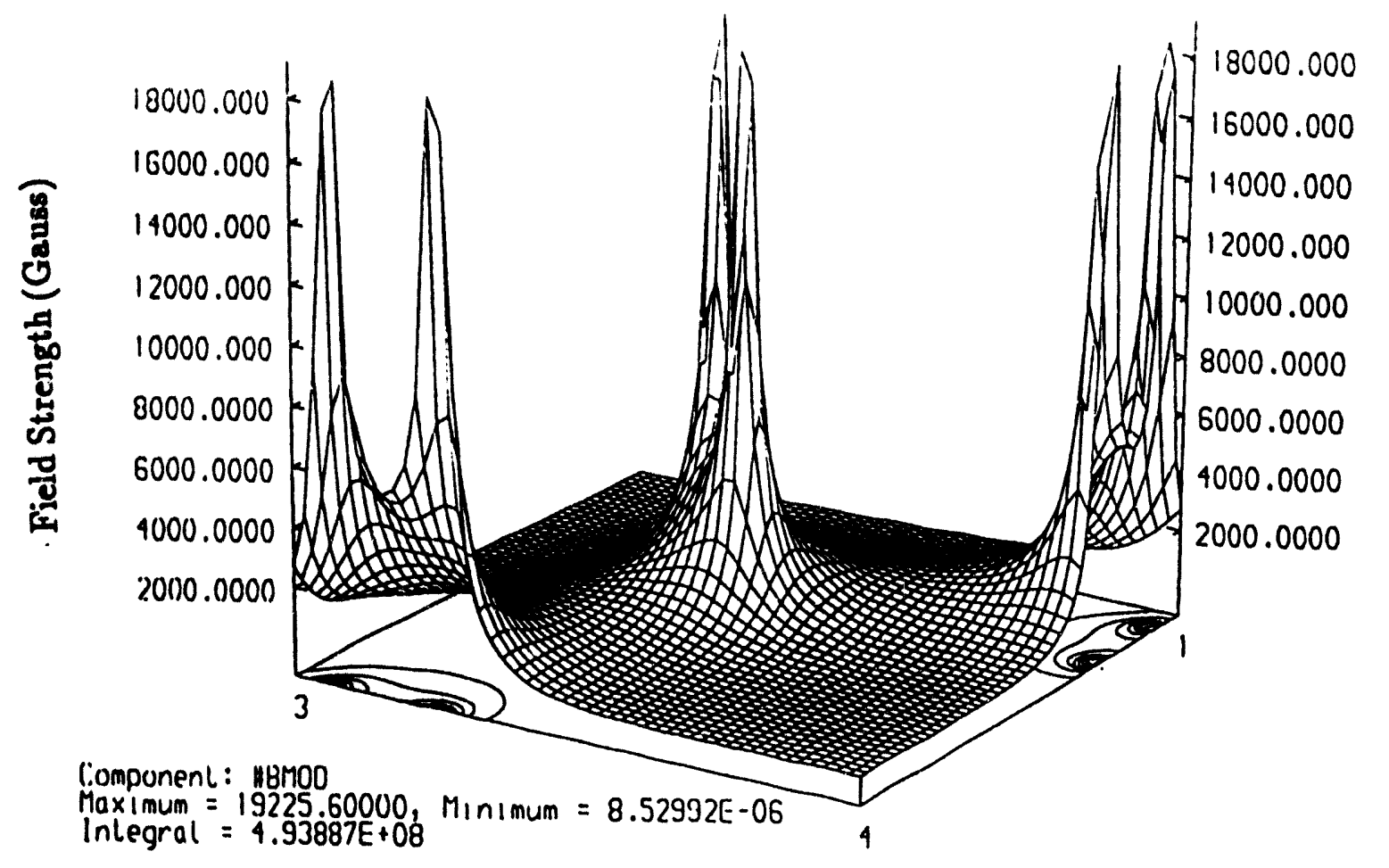
$3=7.301 E-06$ 600.00000
$4=0.0$
0.0
carlesian
$=600.00000$
0.0

$2=849.59900$

44.999900

0.0

$3=600.00000$

89.999900

0.0

$4=0.0$

0.0

polar

GRACEBCUR2 .DAT

27/Jan/92 12:35:29 Page $3:$ HIST GRACEBCUR2. OAT HBMOD VIEW=2

$1=600.00000$
0.0
0.0
$2=600.75700$
600.75700
0.0

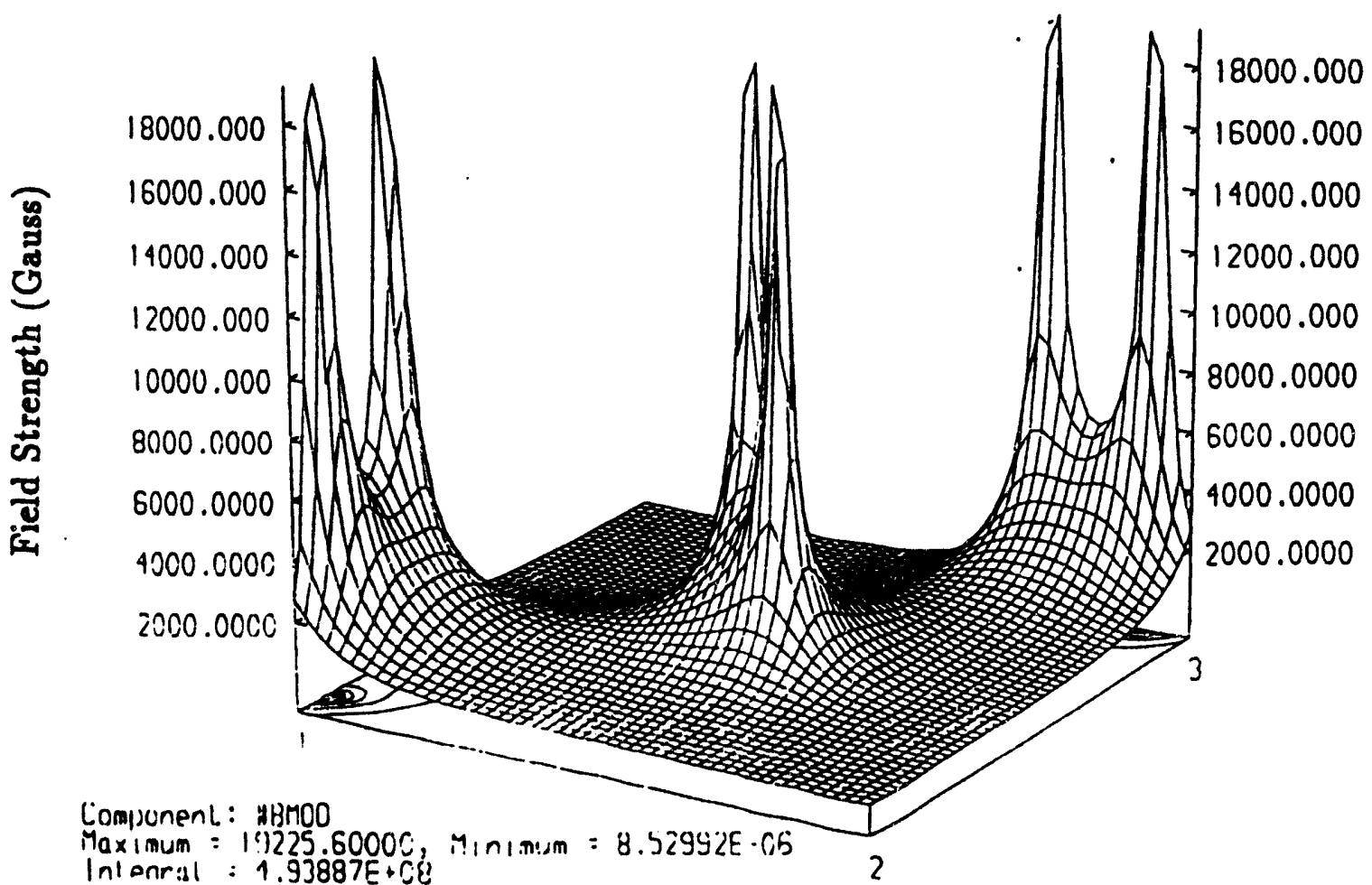

$3=7.301 E-06$ 600.00000

$4=0.0$

0.0

Carlesian

$I=600.00000$
0.0
0.0

$2=849.59900$ 44.999900 0.0

$3=6 C 0.00000$
89.999900

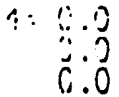

Poigr

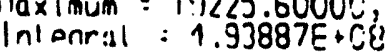

Figure 35 Computed magnetic field magnitude map at mid-axial length, covering three racetracks of the eight racetrack magnet 


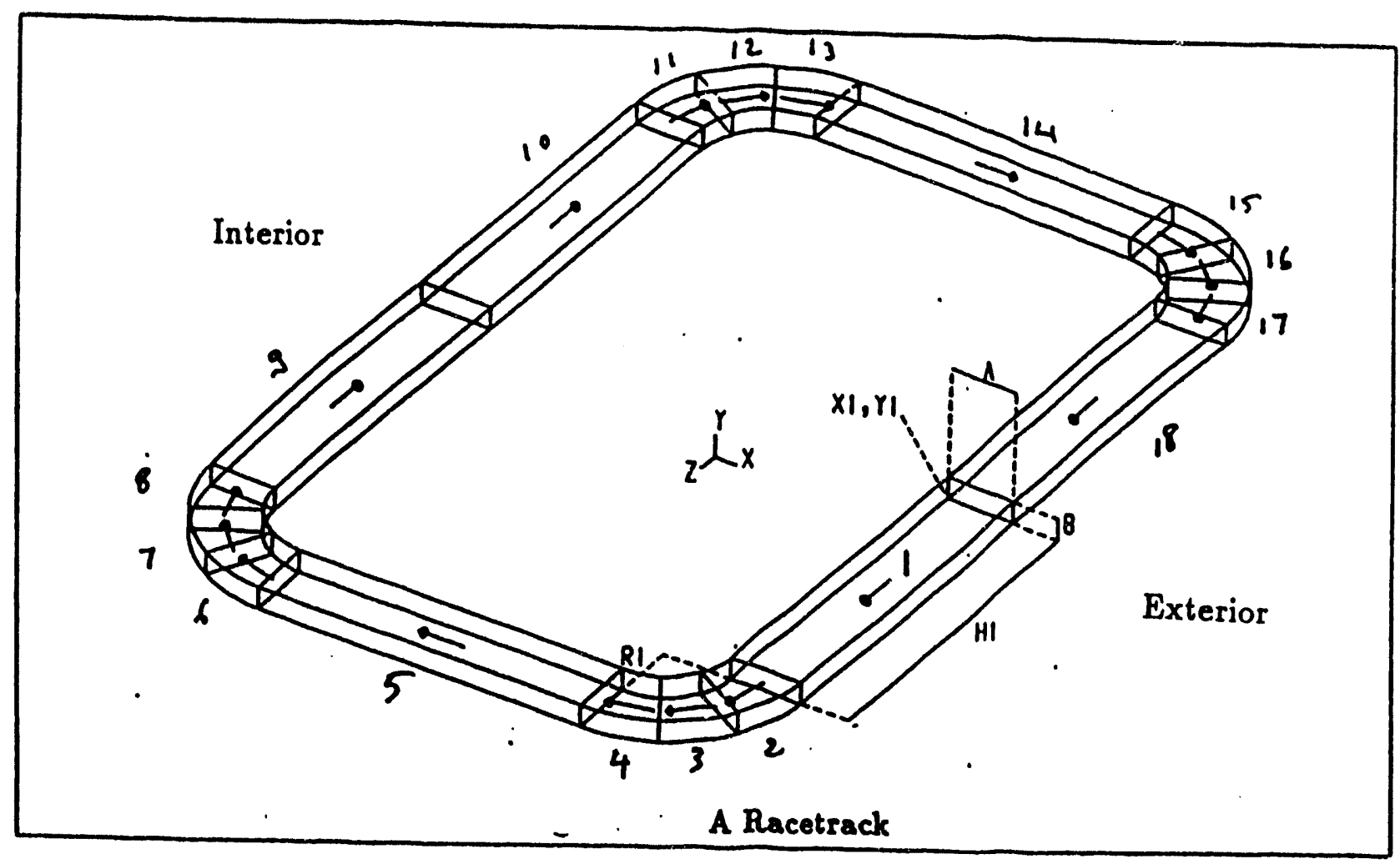

Figure 36 Layout of the 18 internal elements each racetrack 
Table 2 Force calculation on each racetrack of the 6 racetrack configuration

\begin{tabular}{|c|c|c|}
\hline \multirow[t]{2}{*}{ CoIL } & $\begin{array}{r}\text { SECTION } \\
\text { PART } 1 \\
\text { PART } 2 \\
\text { PART } 3 \\
\text { PART } 4 \\
\text { PART } 5 \\
\text { PART } 5 \\
\text { PART } 7 \\
\text { PART } 8 \\
\text { PART } 3\end{array}$ & $\begin{array}{l}\text { Force }(x) \\
1437889.000 \\
43552.00000 \\
36510.30000 \\
12551.70000 \\
0.0 \\
-18483.4000 \\
-56727.6000 \\
-78166.5000 \\
-3999647.00\end{array}$ \\
\hline & $\begin{array}{l}\text { PART } 10 \\
\text { PART 11 } \\
\text { PART i2 } \\
\text { PART is } \\
\text { PART i4 } \\
\text { PART is } \\
\text { PART if } \\
\text { PART if } \\
\text { PART if } \\
\text { ALL }\end{array}$ & $\begin{array}{l}-3999647.00 \\
-78166.5000 \\
-56727.6000 \\
-18483.4000 \\
0.0 \\
12551.70000 \\
36510.30000 \\
43552.10000 \\
1437889.000 \\
-5245042\end{array}$ \\
\hline
\end{tabular}

$\begin{array}{ll}\text { Eorce }(Y) & \text { Eorce }(Z) \\ 0.040085200 & 0.0 \\ -0.00283669 & 12300.50000 \\ 0.004469120 & 36545.20000 \\ 0.017964700 & 44694.00000 \\ -0.00499147 & 382395.0000 \\ -0.00267346 & 65952.80000 \\ 0.004630310 & 56329.40000 \\ 0.018257300 & 21239.80000 \\ 0.047695800 & 0.342595000 \\ -0.01439790 & 0.342595000 \\ -0.00293056 & -21239.8000 \\ 0.004485580 & -56329.4000 \\ 0.017331400 & -65952.8000 \\ -0.00267093 & -382395.000 \\ -0.00299228 & -44694.0000 \\ 0.004158740 & -36545.1000 \\ 0.016920500 & -12300.5000 \\ 0.021979000 & 0.0 \\ 0.164484 & 0.611328\end{array}$

Table 3 Force caiculation on each racetrack of the 8 racetrack configuration

\begin{tabular}{|c|c|c|c|c|}
\hline COIL & SECTION & $\operatorname{Eorce}(x)$ & Eorce $(Y)$ & Eorce (Z) \\
\hline & $\begin{array}{l}\text { PART : } \\
\text { PART } 2 \\
\text { PART } 5 \\
\text { PART } 4 \\
\text { PART } 5 \\
\text { PART } 6 \\
\text { PART } 7 \\
\text { PART } 8 \\
\text { PART } 9 \\
\text { PART } 10 \\
\text { PART } 11 \\
\text { PART } 12 \\
\text { PART } 13 \\
\text { PART } 14 \\
\text { PART } 15 \\
\text { PART } 16 \\
\text { PART 17 } \\
\text { PART } 18 \\
\text { ALI }\end{array}$ & $\begin{array}{l}744222.0000 \\
35273.70000 \\
30492.30000 \\
10196.50000 \\
0.0 \\
-10276.6000 \\
-30752.7000 \\
-35677.5000 \\
-777606.000 \\
-777606.000 \\
-35677.5000 \\
-30752.7000 \\
-10276.6000 \\
0.0 \\
10196.50000 \\
30492.30000 \\
35273.80000 \\
744222.0000 \\
-68256.2\end{array}$ & $\begin{array}{l}1778.650000 \\
24.78880000 \\
19.55240000 \\
9.739222000 \\
55.98920000 \\
2.639370000 \\
-16.1223000 \\
-32.4309000 \\
-2737.29000 \\
-2737.22000 \\
-32.4499000 \\
-16.1223000 \\
2.658245000 \\
55.99410000 \\
9.720674000 \\
19.55280000 \\
24.80750000 \\
1778.640000 \\
-1788.9\end{array}$ & $\begin{array}{l}0.0 \\
10101.30000 \\
30505.90000 \\
35704.00000 \\
234591.0000 \\
35991.60000 \\
30761.90000 \\
10208.10000 \\
0.047461300 \\
0.047461300 \\
-10208.1000 \\
-30761.9000 \\
-35991.6000 \\
-234591.000 \\
-35704.0000 \\
-30505.9000 \\
-10101.3000 \\
0.0 \\
0.11914\end{array}$ \\
\hline
\end{tabular}




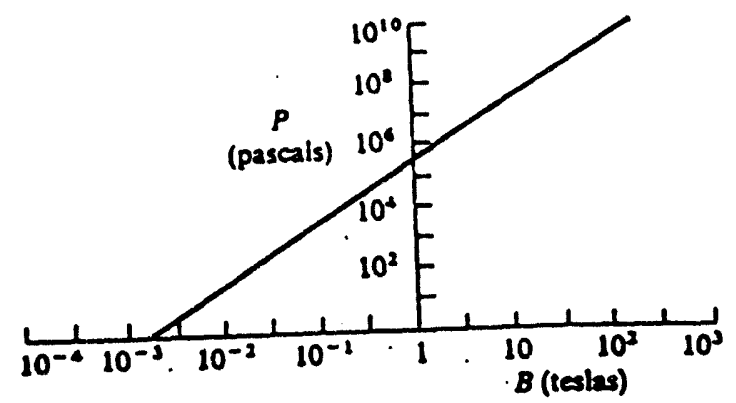

Figure 37 Magnetic pressure $\mathrm{B}^{2} / 2 \mu_{0}$ as a function of $\mathrm{B}$. The magnetic pressure is equal to the magnetic energy density. Atmospheric pressure at sea level is about $10^{5}$ pascals 


\section{4(e.1) Lundquist_Solution}

A single force-free cylindrical solution was proposed by Lundquist (1951). This solution takes the form:

$$
\begin{aligned}
& \mathrm{H}_{2}=\mathrm{AJ}_{0}(\alpha \mathrm{r}) \\
& \mathrm{H}_{\theta}=\mathrm{AJ}_{1}(\alpha r)
\end{aligned}
$$

Where $\mathrm{J}_{\mathrm{O}}$ and $\mathrm{J}_{1}$ are the first order Bessel functions. The field solution is schematically illustrated in Figure 38(a). An implementation on a finite geometry coil was proposed by Furth, et al. (1988) with four helical windings of the varying pitches as shown in Figure 38. For our wodel of the Lundquist coil, only six helical windings are used as shown in Figures 39 and 40.

Before discussing the finite element discretization of the Lundquist solution, let us review the concepts governing this solution. For a field vector $F$, the following equality applies.

$$
\nabla \times \nabla \times F=\nabla(\nabla \cdot F)-(\nabla \cdot \nabla) F
$$

For a field $\mathrm{H}$ with zero divergence, one obtains the equality

$$
\nabla \times \nabla \times \mathrm{H}=-\nabla^{2} \mathrm{H}
$$

In the case of a force free field, where $\nabla \times H=\alpha H=j$, one obtains

$$
\nabla \times \nabla \times \mathrm{H}=-\nabla^{2} \mathrm{H}=\nabla \times(\alpha \mathrm{H})=\nabla \cdot \alpha \times \mathrm{H}+\alpha \cdot \nabla \times \mathrm{H}
$$

and for constant $\alpha$ fields, one obtains the following wave equation:

$$
\nabla^{2} \mathrm{H}+\alpha^{2} \mathrm{H}=0
$$

which becomes without loss of generality

$$
\nabla^{2} \mathrm{H}+\mathrm{H}=0 .
$$

We are seeking a field which has the following characteristics

$$
\mathrm{H}=\left(0, \mathrm{H}_{\theta}, \mathrm{H}_{\mathrm{z}}\right) \text { with } \mathrm{H}=\mathrm{H}(\mathrm{r}) \text {. }
$$


$\mathbf{a}$

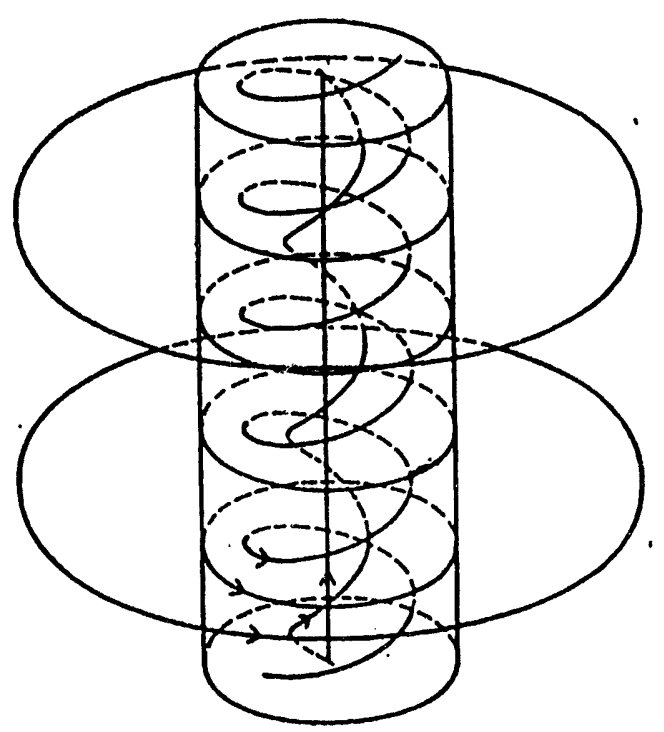

b

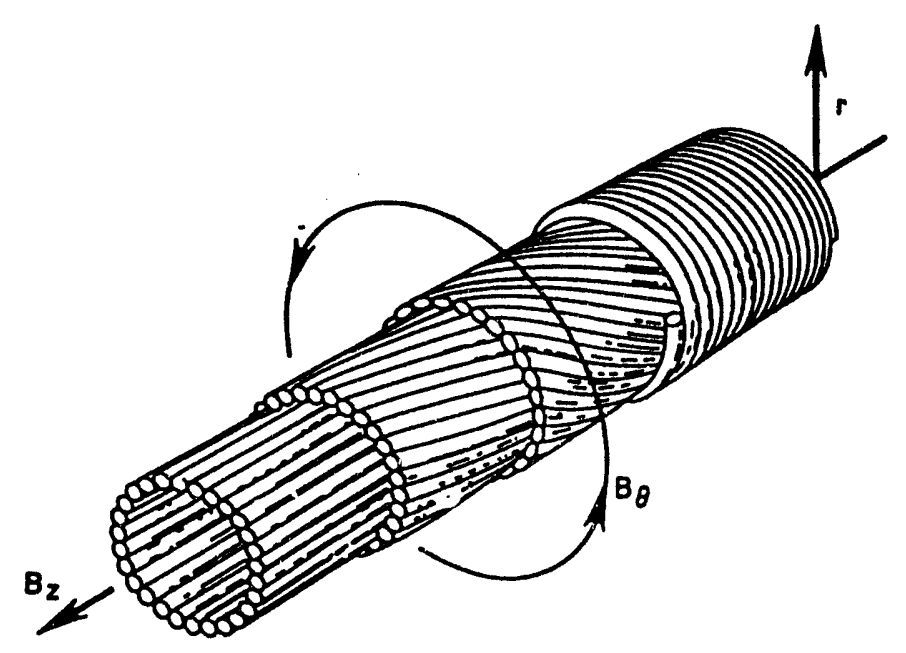

Figure 38 a) Lundquist force-free mathematical solution in an infinite medium, b) typical applications of Lundquist solution for a cable of four helical windings of varying pitch (Furth, et al., 1988) 


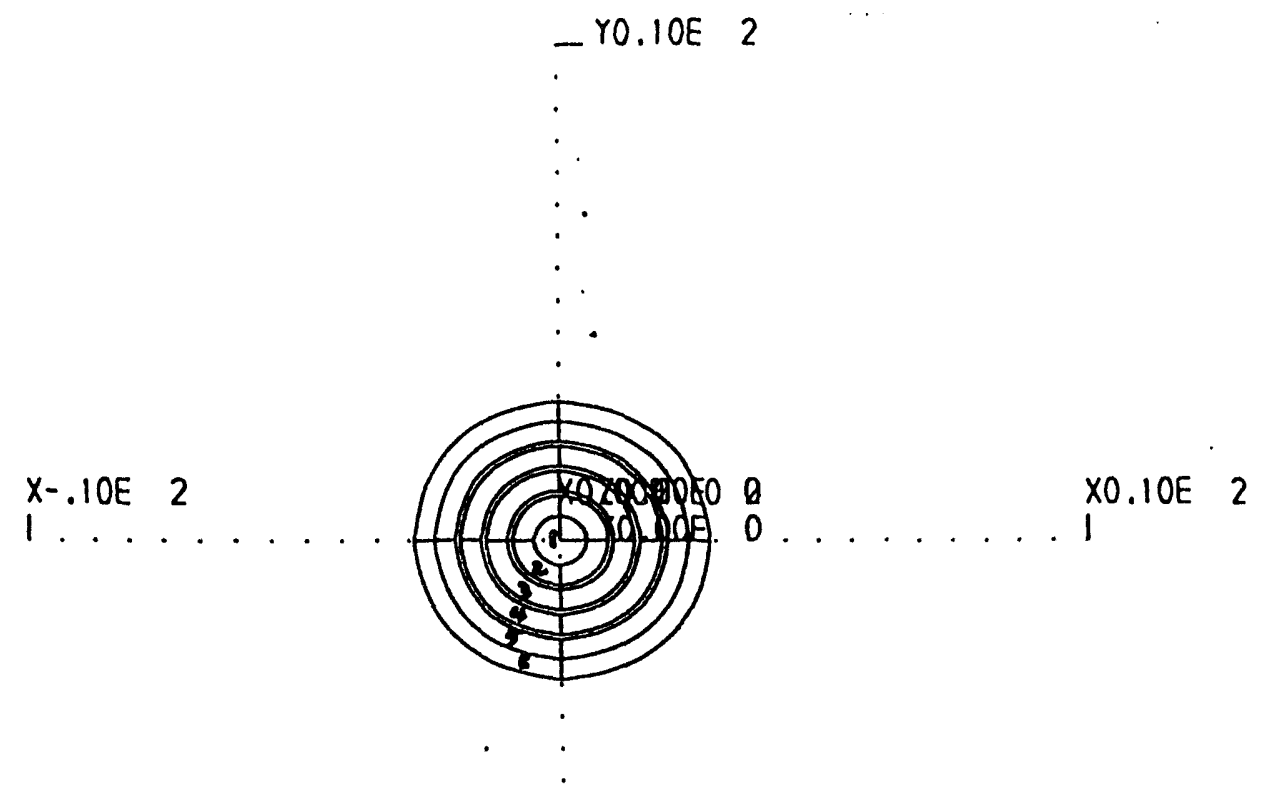

Figure 39 Model of the force-free Lundquist coil layout 
PO. 0 2
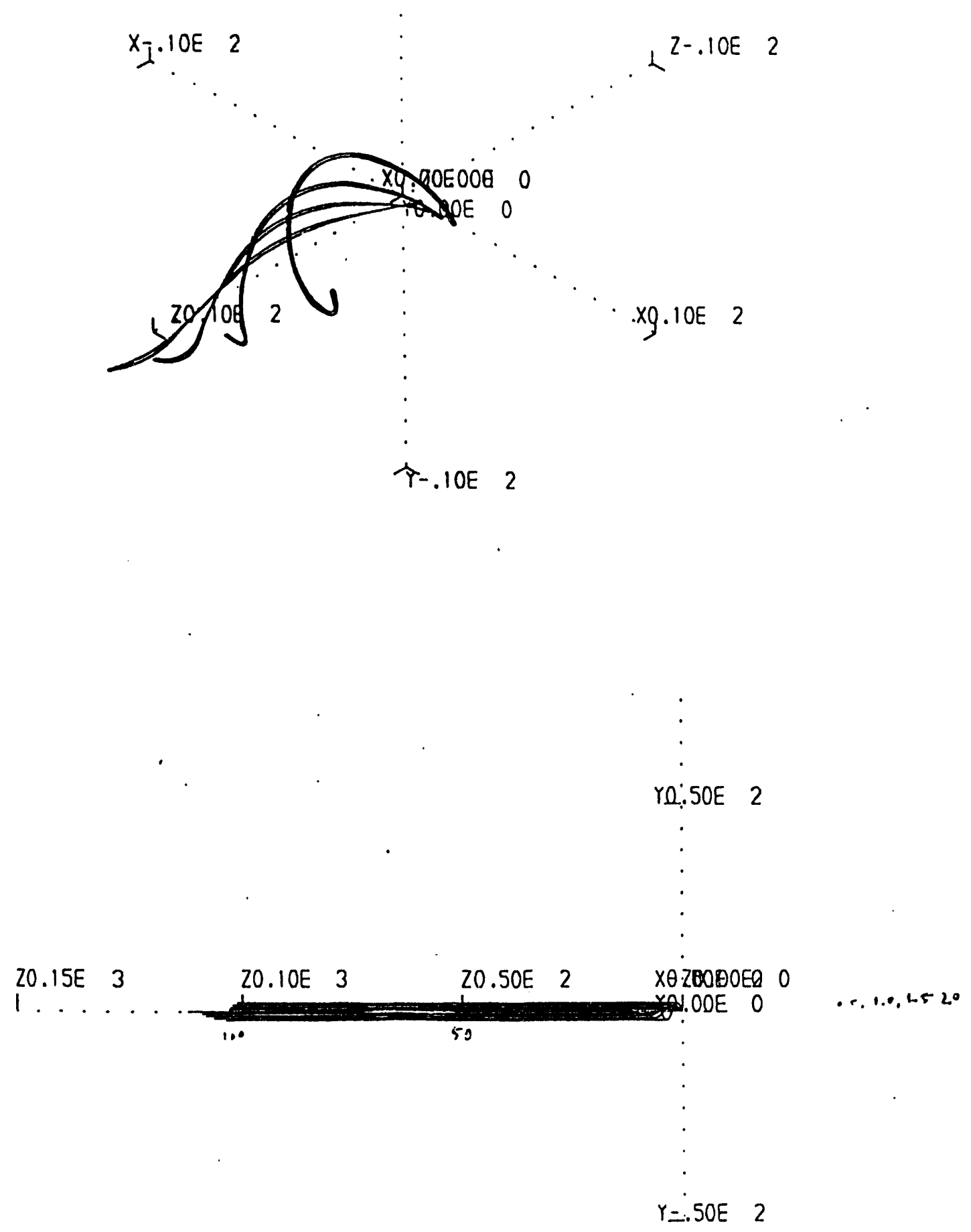

Figure 40 Top: Helix generator for the Lundquist solution.

Bottom: Six nested cylindrical helicoidal conductors 
The wave equation defined above thus becomes

$$
\begin{aligned}
& \frac{1}{\mathrm{r}} \frac{\partial}{\partial \mathrm{r}}\left(\frac{\partial \mathrm{H}_{\mathrm{z}}}{\partial \mathrm{r}}\right)+\mathrm{H}_{\mathrm{z}}=0 \\
& \frac{\partial}{\partial \mathrm{r}}\left(\frac{1}{\mathrm{r}} \frac{\partial}{\partial \mathrm{r}}\left(\mathrm{rH}_{\theta}\right)\right)+\mathrm{H}_{\theta}=0 .
\end{aligned}
$$

Equations 29 and 30 may be recast in the following form:

$$
r^{2} \frac{\partial^{2} H_{\theta}}{\partial r^{2}}+r \frac{\partial H_{\theta}}{\partial r}+H_{\theta}\left(r^{2}-1\right)=0
$$

and

$$
\mathrm{r}^{2} \frac{\partial^{2} \mathrm{H}_{\mathrm{z}}}{\partial \mathrm{r}^{2}}+\mathrm{r} \frac{\partial \mathrm{H}_{\mathrm{z}}}{\partial \mathrm{r}}+\mathrm{r}^{2} \mathrm{H}_{\mathrm{z}}=0
$$

As can be seen Equations 31 and 32 are special cases of the more general Bessel's differential equations which read

$$
\mathrm{r}^{2} \mathrm{H}^{\prime \prime}+\mathrm{rH}^{\prime}+\left(\mathrm{r}^{2}-\mathrm{n}^{2}\right) \mathrm{H}=0
$$

whose solutions are

$$
J_{n}(r) \text { and } J_{-n}(r) \text { for } n \text { nonintegers }
$$

and

$$
J_{n}(r) \text { and } Y_{n}(r) \text { for } n \text { integers }
$$

Since $Y_{n}(0)$ is unbounded, the solution field becomes

$$
\begin{aligned}
& H_{\theta}=a J_{1}(\alpha r) \\
& H_{z}=b J_{0}(\alpha r)
\end{aligned}
$$




\section{4(d.2) Verification of the Force-Free Conditions}

It is known that the solutions of the wave equations are not necessarily the solutions of the force-free field equations. To show that solutions 34 and 35 are indeed force-free let us calculate $\nabla \times \mathrm{H}$

$$
\nabla \times \mathrm{H}=\left(0, \mathrm{hJ}_{1}, \mathrm{iJ}_{0}\right)
$$

which equals to $\mathrm{H}$ if and inly if $\mathrm{a}=\mathrm{b}$. Hence, the force-free solution becomes

$$
\mathrm{H}=\left(0, \mathrm{~J}_{1}, \mathrm{~J}_{0}\right)
$$

\section{4(d.3) Boundary Conditions}

We are interested in a force-free solution within a bounded region, say $r$ in $[0, a]$. In this region, the force-free field is governed by the wave equation $\nabla^{2} \mathrm{H}+\alpha^{2} \mathrm{H}=0$, but outside this region, where no currents exist, the wave equation becomes $\nabla^{2} \mathrm{H}=0$. The exterior fields is thus governed by the following equation:

$$
\frac{\partial}{\partial r}\left(\mathrm{rH}_{\theta}\right)=\mathrm{kr}
$$

but at $r=\infty$ the field must be bounded, thus $k=0$, and $H_{\theta}$ varies as $1 / r$. For $H_{z}, r \frac{\partial}{\sigma r}\left(H_{z}\right)$ should also be constant, $k$, so the field varies as $k \ln r$. Since the field cannot become infinite at $r=\infty$, thus $\mathrm{k}=0$, and the field $\mathrm{H}_{\mathrm{z}}$ is zero throughout the external region. The external field is then represented by

$$
H=(0, k / r, 0)
$$

In the interior region, where the conductor is present $(\alpha \neq 0)$ force-free solution is still represented by

$$
\mathrm{H}=\left(\mathrm{U}, \mathrm{J}_{1}, \mathrm{~J}_{0}\right)
$$

boundary conditions at $\mathrm{r}=0$ are implicit in the sense that they are somewhat given by the Bessel functions. They are:

$$
\mathrm{H}_{\theta}(0)=0
$$




$$
\frac{\partial H_{z}}{\partial r}(0)=0
$$

The boundary conditions at $\alpha r=a$ are given by $H_{\theta}(a)=H_{i}=\frac{k}{a}$

and $\mathrm{H}_{\mathrm{z}}(\mathrm{a})=\mathrm{J}_{0}(\mathrm{a})=0 . \mathrm{H}_{\mathrm{i}}$ denotes the interface field.

\section{4(d.4) Force Calculations on the Lundquist Force-Reduced Coil}

To calculate the forces on the conductors, one may use the Maxwell stress tensor

$$
\mathrm{T}=\frac{1}{2} \mathrm{H}^{2} \mathrm{I}-\mathrm{HH}
$$

In the radial direction, the force is

$$
\mathrm{F}_{\mathrm{r}}=(\nabla \cdot \mathrm{T})_{\mathrm{r}}=\frac{1}{\mathrm{r}} \frac{\partial}{\partial \mathrm{r}}\left(\mathrm{r} \mathrm{T}_{11}\right)-\frac{1}{2} \frac{\partial}{\partial \mathrm{r}} \mathrm{T}_{12}-\frac{1}{\mathrm{r}} \mathrm{T}_{12}-\frac{1}{\mathrm{r}} \mathrm{T}_{22}+\frac{\partial \mathrm{T}_{13}}{\partial \mathrm{z}}
$$

where

$$
\begin{aligned}
& T_{11}=1 / 2 B^{2} \\
& T_{12}=0 \\
& T_{22}=\frac{1}{2}\left(H_{z}^{2}-H_{x y}^{2}\right) .
\end{aligned}
$$

The radial force becomes

$$
\begin{aligned}
& F_{r}=\frac{1}{r}\left(T_{11}-T_{22}\right)+\frac{\partial T_{11}}{\partial r} \\
& =\frac{1}{r} H_{x y}{ }^{2}+\frac{1}{2} \frac{\partial H^{2}}{\partial r} .
\end{aligned}
$$

Where $\mathrm{H}_{\mathrm{xy}}$ represents the azimuthal field magnitude in the $\mathrm{xy}$ plane.

\section{4(d.5) Examples}

As example, one may take the Bessel function representation of the force-free field. In this case, the radial force $F_{r}$ given (46), becomes 


$$
\begin{aligned}
F_{r} & =\frac{J_{1}^{2}}{r}+\frac{1}{2} \frac{\partial J_{0}^{2}+J_{1}^{2}}{\partial r} \\
& =\frac{J_{2}}{r}+J_{1}\left(J_{0}-\frac{J 1}{r}\right)-J_{0} J_{1}=0
\end{aligned}
$$

indicating that the field is indeed force-free. Note that this results is independent of the truncation radius chosen. The forces acting on external regions can be similarly evaluated by

$$
F_{r}=\frac{k^{2}}{r^{3}}-\frac{k}{r} \frac{k}{r^{2}}=0
$$

here again no radial forces are exerted in the outer domain. Hence, the solution given by Equations 39 an $\mathrm{d} 40$ is force-free in both the interior and exterior domains.

\section{4(d.6) Compressive Forces in a Conventional Cylindrical Conductor}

If a longitudinal cylindrical conductor is used to create an azimuthal magnetic field $\mathrm{Hi}$ at the surface of the conductor, the field inside the conductor is given by

$$
H_{\theta}=H_{i}\left(\frac{r}{r_{i}}\right) \text {, for, } r \text { in }\left[0, r_{i}\right]
$$

The force exerted inside the conductor is given by Eq. 46 or

$$
F_{r}=\frac{H^{2} r}{r_{i}^{2}}+\left(\frac{H_{i}}{r_{i}}\right)^{2} r=2\left(\frac{H_{i}}{r_{i}}\right)^{2} r \neq 0 .
$$

We see that for a conventional wire, a non-zero internal force is exerted in the interior of the conductor. The maximum radial force takes place on the surface of the conductor $\left(r=r_{1}\right)$, the force being equal to $2 \mathrm{Hi}^{2} / \mathrm{r}_{\mathrm{i}}$ (force per unit of volume). Note, on the other hand, that for the same external field $\mathrm{H}_{\mathfrak{i}}$, the Lundquist solutions indicates that no forces reside inside the conductor (Eq. 48).

To gain further understanding on how much force reduction can be accomplished using a force-free coil made of six helical windings, a three-dimensional computer finite-element model analysis is performed in the following section by using the TOSCA computer code.

\section{4(d.7) Finite-Element Computer Modeling of Force-Free Fields}

A discretization of the Lundquist's solution is presented below for which six evenly spaced concentric cylindrical conductors were chosen to follow the helical system described by Equations 
(21) and (22). The helix generators are shown in Figures 39 and 40 (top) with the final six cylindrical conductors shown below (Figure 40 bottom). In the numerical simulations, $A$ in Equations (1) and (2) is assumed to be unity and $\alpha$ r varies from 0 to 10. The Lundquist conductor is truncated at $\alpha \mathrm{r}=2.5$, value for which $\mathrm{J}_{0}(\alpha r)$ becomes zero.

To solve this problem, each conductor was defined by four conducting elements to limit the total number of conductors under 100 . Such crude resolution of the conductors was initially attempted on the VAX, but it took 10 hours of VAX CPU just to compute, on a very crude mesh the field generated by the six helices. A decision was made to use the ANL CRAY supercomputer to accelerate the resolution of this problem.

Figure 41(a) provides a schematic representation of the computer model for the coils with the three-dimensional finite element mesh on which magnetic field calculations are performed. The fields are computed only in this mesh, which limits computation running time. As can be seen in Figure 41 (b), the computed field is cylindrical with a preferential field orientation pointing towards the $z$ axis near the $z$ axis center. In Figure 42, two histograms of the magnetic field strength are presented which show a continuous decreasing function of the field as $r$ increases. In Figure 43 (top), the azimuthal strength of the field is presented and qualitatively follows the theoretical $\mathrm{J}_{1}$ Bessel function which is presented in Figure 45. It has a maximum at $r=1.56(x=1.1, y=1.1)$ and decreases as $r$ increases beyond $r=1.6$. The total field strength is shown at the bottom of Figure 43. It shows that at $x=1.8(r=2.5)$ the field is about 1000 Gauss, which is about half the central value of the field. This result is consistent with the Lundquist solution which predicts a field reduction of about $50 \%$ at the first zero of the Bessel function Jo (see Figure 45). In Figure 44 , the poloidal field is represented. As can be seen beyond $r=2.8$ or $x=y=2.0$, the poloidal field is zero and that is indeed what is expected with a truncated Lundquist solution.

\section{(4.d8) Radial Force Comparisons}

By using Equations 46 and 50, one can estimate and compare the radial forces exerted between a force-reduced and a conventional coil. At $r=2.5$ ( $\alpha$ is set to unity), the inward compressive force exerted at the periphery of the force-reduced coil is about $25 \%$ smaller than that for a conventional wire of same radius. To physically understand how forces are reduced in a force-free winding, one can first look at the field distribution created by a conventional straight

cylindrical wire. The field exterior to the wire varies as $\frac{1}{r}$, and the field interior to the wire varies linearly with the radius $r$. At the conductor surface the field is continuous, but there is a discontinuity in the field gradient, which results in a force compressing the wire. In a force-free winding, the helical coils produce a poloidal field inside the conductor. This field build-up is 
a

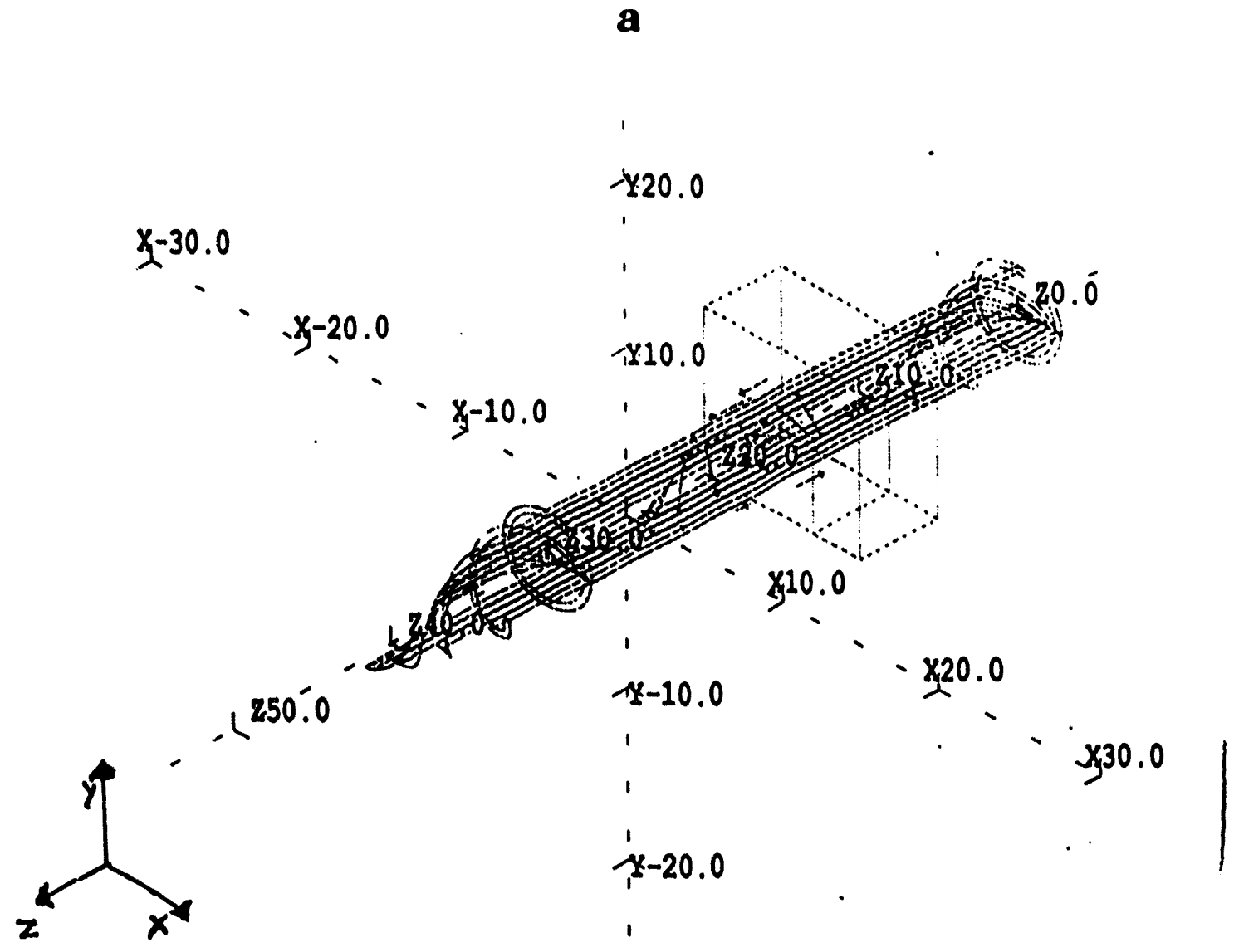

b

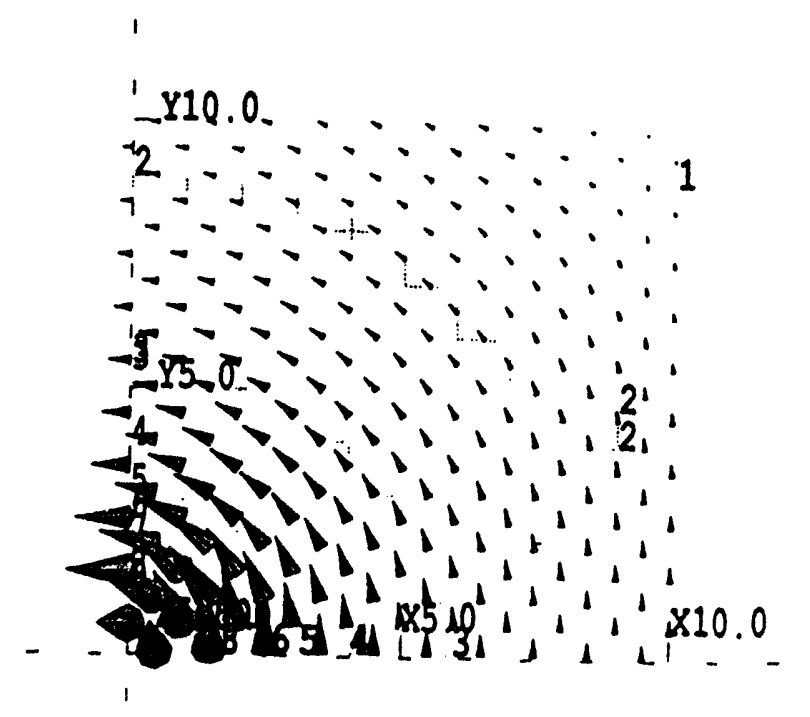

Figure 41. a) Schematic representation of the computational mesh' used around the riested conductors.

b) Computed magnetic field vector in the $x y$ plane 
la . A

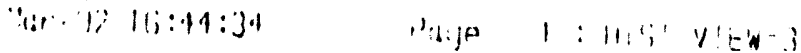

$:: 0 . i 100000$

.1000000

17.000000

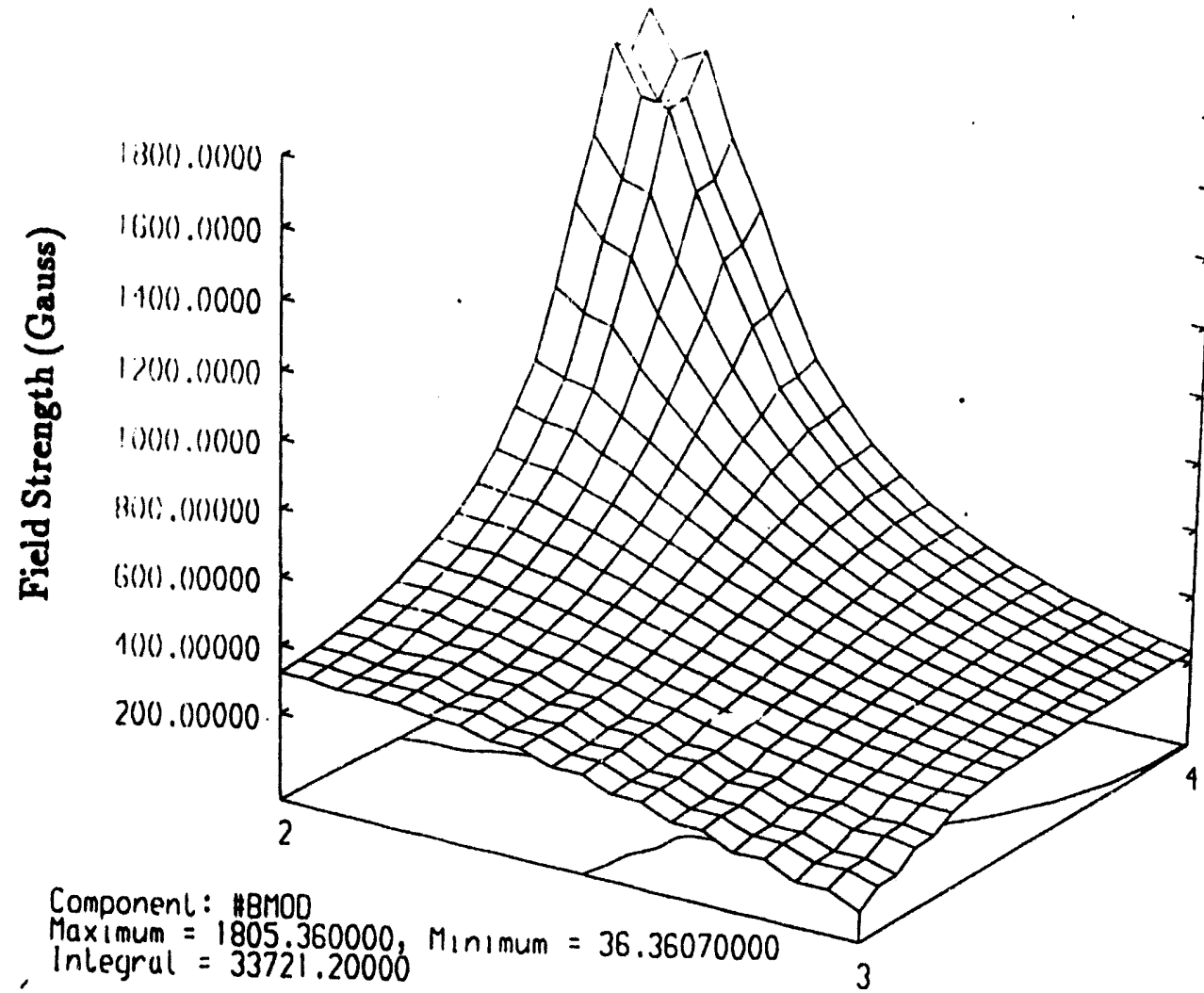

$2=3.04 c 000$ 0.1000000

$\left\{\begin{array}{rr}1800.0000 & 17.000000 \\ 1600.0000 & 3=9.0000000 \\ & 9.0000000 \\ 17.000000\end{array}\right.$

1400.0000

1200.0000

$1=0.1000000$

9.0000000

17.000000

1000.0000

Carlesian

800.00000

600.00000

400.00000

200.00000

$1=0.1414210$

44.999900

17.000000

$2=9.0005550$

0.6365930

17.000000

$3=12.727900$

44.999900

17.000000

$4=9.0005550$

89.363400

17.000000

Polar

BESCART .OAT

4/Mar/92 16:44:11 Page 3: HIST VIEW=2

$1=0.1000000$

0.1000000

$2=9.0000000$

0.1000000
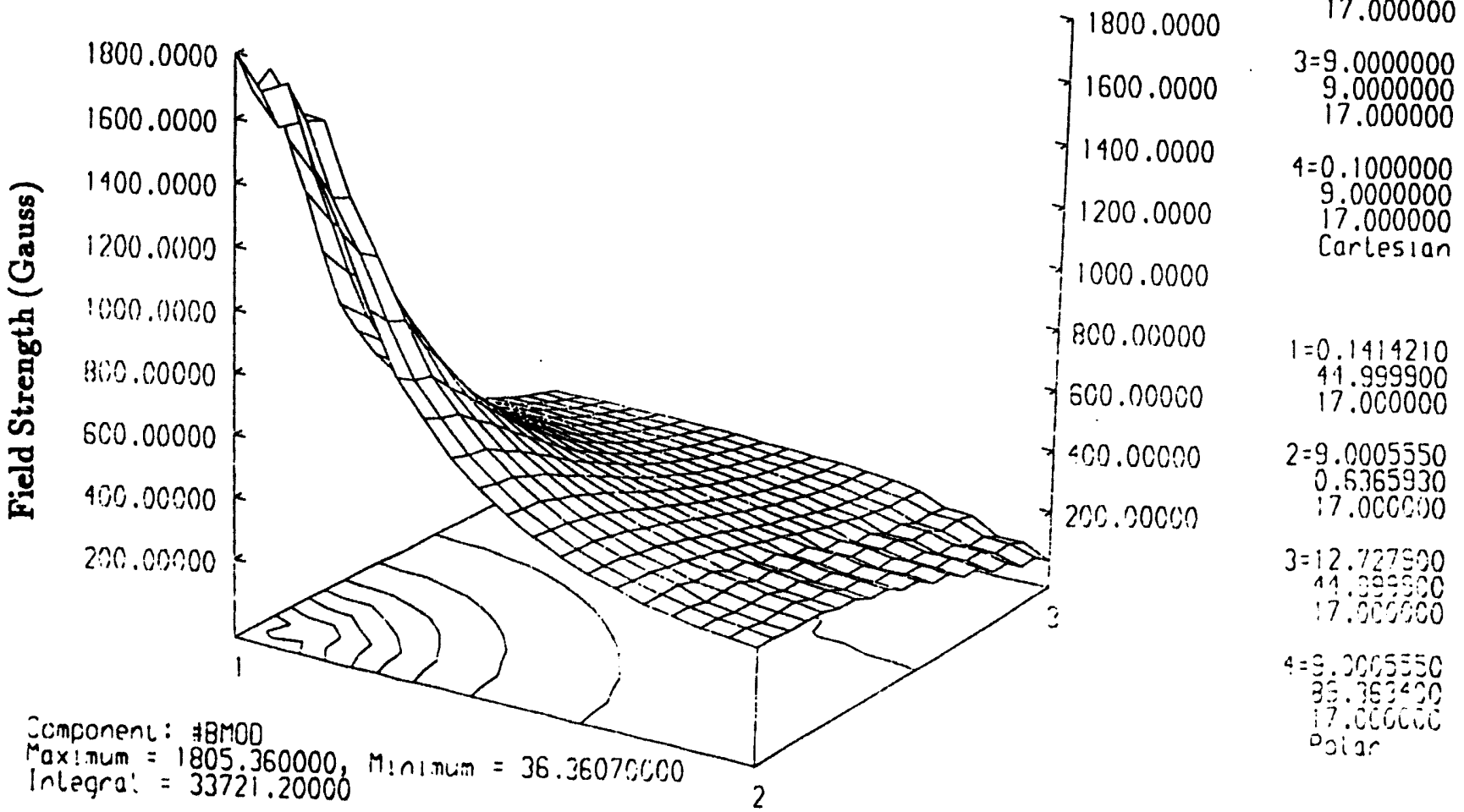

Figure 42 Histogram of the magnetic field strength in a xy cross-section plane 

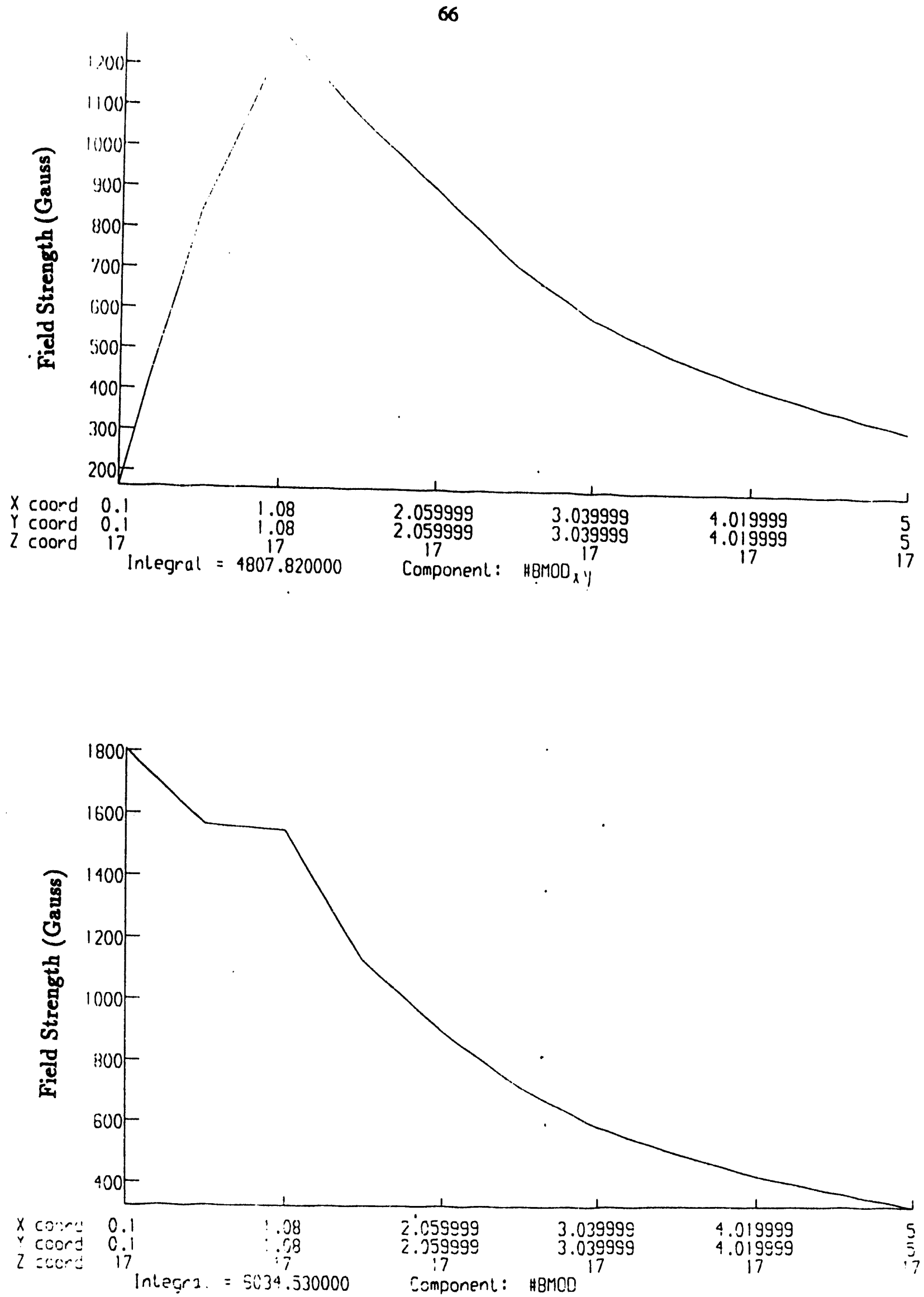

Figure 43 Top: Azimuthal field strength in the xy cross-sectional plane; Bottom: total field strength (azimuthal + poloidal) in the xy cross-section plane 


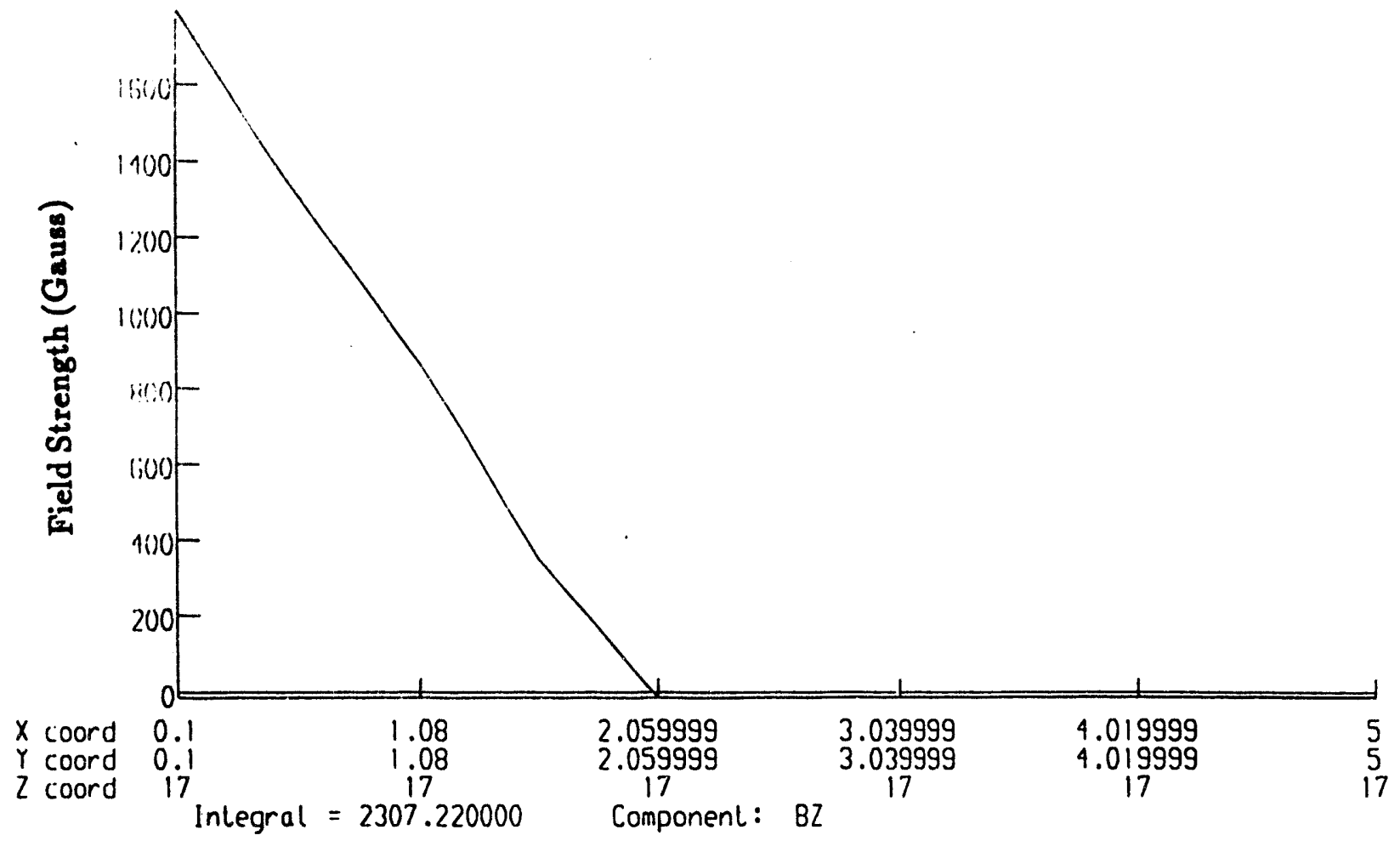

Figure 44 Poloidal field strength on the xy cross plane

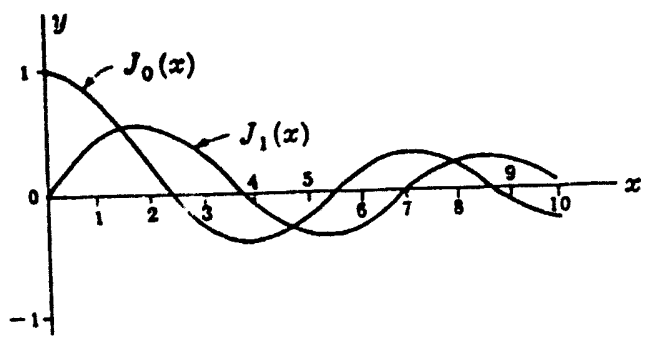
Figure 45 Theoretical poloidal (Jo(n)) and azimuthal $(\mathrm{Jl}(\mathrm{n}))$ field strengths calculated by Besses
functions for the Lundquist coil (infinite domain) 
analogous to a pressure build-up which would tend to compensate the internal compressive force. It is this force compensation which brings a global reduction of the internal compressive force on the wire.

These encouraging results are only preliminary. They show that force-reduction is indeed possible by using truncated force-free solutions. An optimization study in which the discretization of the Lundquist coil is made finer should be carried out to possibly establish larger force reduction. Because of time constraints, this optimization study could not be carried out. Howęver, it is believed that a more thorough understanding of force-reduced fields for discrete coil geometries was provided by this study.

Quite an intriguing comment can be made that would need further investigation. It has been shown that the ideal Lundquist coil displays no forces in its interior and its exterior domains. However, nothing has been said about the coil periphery. To satisfy the virial theorem, a surface force should be present at the periphery of the coil. We also know that although tiie Maxwell stress tensor is continous at the interface, its derivatives are discontinuous. This leads to surface forces which could not be calculated by the procedure followed in this study. It is speculated, however, that this surface force is responsible for the low force reduction (25\%) found in this study. Further work is needed to provide clear understanding on the nature and strength of the forces exerted at the periphery of the force-reduced coil. 


\section{5 .0 Needs and Directions for Future R\&D}

As indicated in this report, the development of analytical closed form solutions for forcefree fields is worth pursuing. At the same time, the problem should also be tackled numerically for a variety of boundary conditions and coil geometries. As indicated in this preliminary study, a substantial amount of computer time is necessary to solve even the simplest problem. It is, therefore, anticipated that the entire discretization and field computation for complex coils may require advanced and highly performant supercomputers and computational algorithms. In this study, we purposely ignored the design influences of force-free concepts upon the complexity of winding and cryogenic design. As stated in Appendix A, the design of an advanced superconducting magnet not only requires field analyses, but also structural, thermal, and conductor analyses. It is only within this envelope of constraints, that benefits from force-free field concepts can be assessed.

On the same front, small-scale experimental magnets designed with force-free concepts should be built and tested. This step is important to validate the analytical and computer models and gain physical understanding of the practical limits of such concepts. 


\subsection{Conclusions}

In this study several new force-reduced magnet configurations were reviewed and studied. A helicoidal torsatron, a rectangular torsatron and a truncated Lundquist coil were analyzed numerically. Significant force reduction was found on the windings of these systems. However, the field homogeneity/uniformity generally becomes worse than those generated by conventional magnets. Other disadvantages of such magnets namely the complexity of their windings, design, construction, maintenance, and repair have to be considered. Some of these considerations may outweigh the possible weight reduction benefit that could be accrued by using force-free field concepts. From this preliminary analysis, it is perceived that a tremendous research and development effort will be required to design and optimize these new coil configurations. This study clearly shows that force-reduced magnet configurations are possible and grossly follow the trends of proposed theoretical models. It remains to refine these models, show that larger force reductions are possible and that such magnets configurations can find a use for various technological applications. 


\subsection{Acknowledgments}

This research was funded by an LDRD grant, proposed by G.E. Marsh, M. Petrick, A. Wolsky, and R. Poepple, and approved by the Energy, Environmental, and Biological Research ALD Office. Frank Degges is thanked for his help on magnetic field computations for the racetrack geometries. G.E. Marsh, M. Petrick, A. Wolsky, R. Poeppel of ANL and J. Schwartz of the University of Illinois are warmly thanked for their constructive comments and discussions during this work. 


\section{Appendix A: \\ Review of Superconducting Magnet Design Procedures}

If weight reduction of a superconducting magnet by force-free concepts is sought, not only has the ciesign of the coils to be modified to take advantage of the structural weight reduction, but in addition the whole magnet design may have to be revisited to accommodate these new coil configurations.

The design of the superconducting magnet usually starts by the definition of the characteristics of the magnet which are the magnetic field distribution, flux density, configurations volume, uniformity, leakage's, etc. (as shown in Figure A-1). Then, these requirements lead to the identification of the winding configurations with the specification of current densities. This type of study of requirements is called the Field Analysis. Then these conductor configurations have to hold together and may require the specification of the underlying structure of the magnet. This study is called the structural stress analysis, which takes into account the gravity, seismic, and the electromagnetic forces. To maintain the coil in a superconducting state, a thermal analysis is necessary to assess heat losses due to cool-down procedures, field charging, conduction and radiation. These heat loss mechanisms mainly deal with solid, liquid, and gas conductions, convection, radiation between the coils at low temperatures and their shields, and Joule effects on the coils and current leads. After these heat losses are evaluated, the cryostat requirements and design can be established. Once the field, stress and thermal analyses are performed, the specific design of the conductor with its stability analysis are performed. This analysis will set requirements and specifications on the power supply syctem, the protection system and current leads. In designing a superconductor magnet, one has to keep in perspective the guidelines listed in Table A-1. The second item "simplicity of winding design" is important to those who want to devise new windings as those proposed for force-free/reduced coils. An economic cost benefit analysis has to be ultimately performed to assess the economic gains proposed by force reduced coils. 


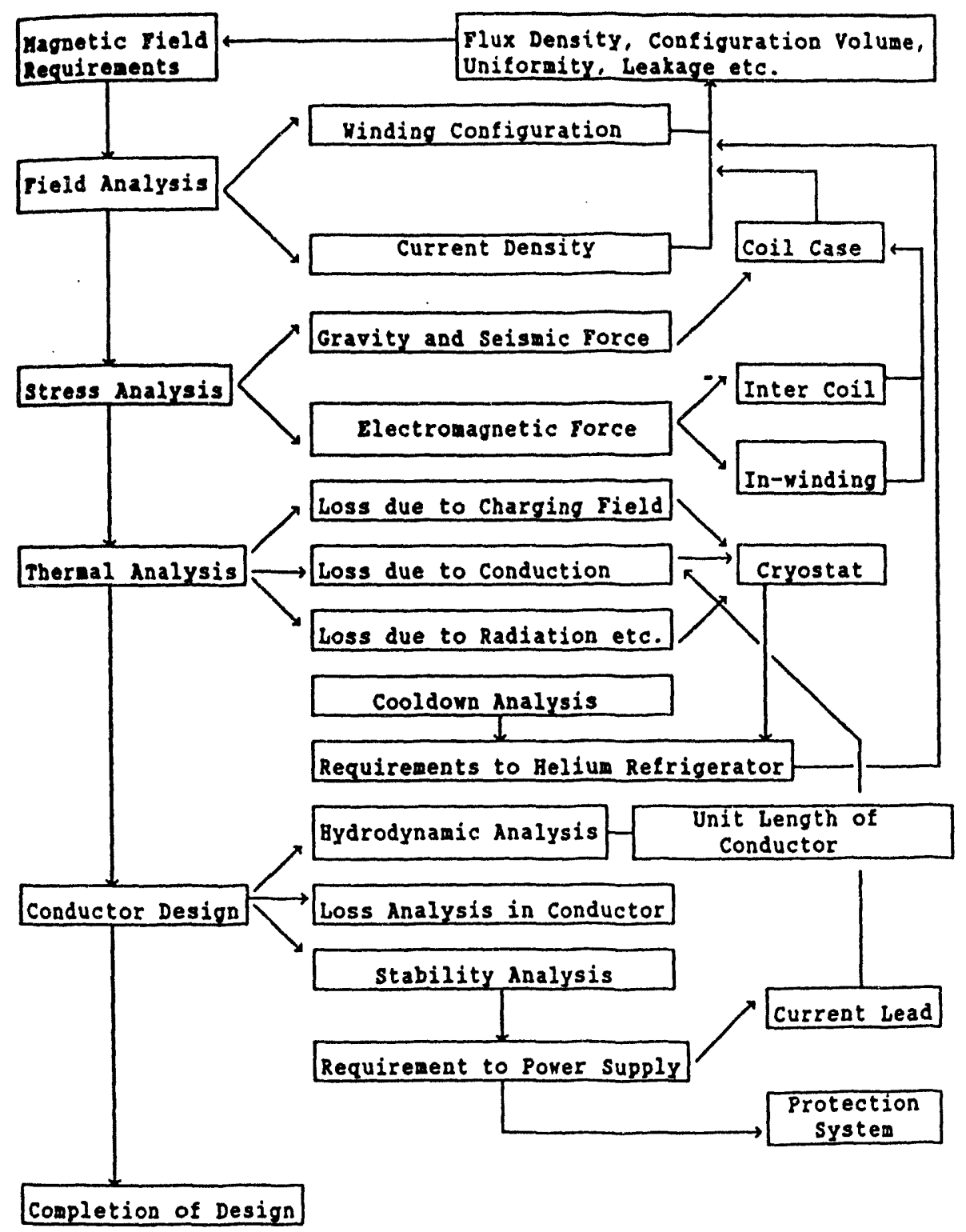

Figure A-1 Typical design procedure for the development of superconducting magnets (M. Akiyama) 
Table A-1:

Practical Considerations for Superconducting Magnet Design

Simplicity of conduct or design

Simplicity of winding design

Cost of conductor fabrication

Cost of winding fabrication

Expenses for quality assurance

Possibility of internal shorts

Vacuum tightness

Thermal efficiency during steady refrigeration

Stiffness of the winding

Possibility of internal disturbance

Stability against external heat input

Self-recovery from normal state

Pressure increase due to fast discharge

Reliability in case of cryogenic system down

Magnetic environmental pollution

As can be seen from Figure A-1 and Table A-2, the final design of the magnet hinges on several analyses that are all interdependent. Hence if one wants to apply a new winding concept, strong repercussions may ensue in the stress, thermal and conductor design analyses. A savings in one particular area may not mean a final savings especially if it is counter-balanced by an increase in complexity and cost in other areas.

\section{Basic Design Considerations for Superconducting Magnets}

\section{(1) Cooling}

One of the basic selection to be done for the coil cooling is the type of cooling. The major types of cooling are the pool-boiling cooling $(\mathrm{PBC})$, force-flow cooling $(\mathrm{FFC})$ and the superfluid cooling (SFC). Among them, SFC provides low operating temperature of $1.8 \mathrm{~K}$ and the critical current of superconductors and heat transfer characteristics are highly improved at this temperature when compared to those at $4 \mathrm{~K}$. However, the thermal efficiency for the refrigeration at $1.8 \mathrm{~K}$ is low and SFC is not suitable for the application to the large magnet systems. Therefore, the actual cooling selection for large coils is PBC or FFC. 
(2) Winding current density

Present status of achievements on the winding current density is shown in Figure A-2. The upper limits of current density for reliable design based on the present technology are 25-30 $\mathrm{A} / \mathrm{mm}^{2}$ for $8-\mathrm{T} \mathrm{Nb} \mathrm{Ti}$ toroidal coils and $40 \mathrm{~A} / \mathrm{mm}^{2}$ for $12-\mathrm{T} \mathrm{Nb} 3 \mathrm{Sn}$ toroidal coils.

(3) Practical superconducting materials and conductor design

Practical superconducting materials to be used in large scale coils include NbTi and NbTiTa for alloy conductors, and $\mathrm{Nb}_{3} \mathrm{Sn}$ and ( $\left.\mathrm{NbTi}\right)_{3} \mathrm{Sn}$ for chemical compound conductors. Relationships between critical current density and magnetic field for such conductors are shown in Figure A-3. A field of $12 \mathrm{~T}$ is possible for a NbTiTa conductor in the reduced temperature region below $1.8 \mathrm{~K}$. The reliability of a $\mathrm{Nb}_{3} \mathrm{Sn}$ coil, however, was recently reported. $\mathrm{Nb}_{3} \mathrm{Sn}$ conductors could be applied to toroidal coils to generate fields greater than $10 \mathrm{~T}$. Note that the typical following characteristics of the superconducting materials have to be considered when designing a superconductor coil.

(1) Critical current density of multifilamentary $\mathrm{Nb}_{3} \mathrm{Sn}$ as a function of temperature and magnetic field. For example, between 2 and $6 \mathrm{~K}$, the critical current density follows the formula.

$\mathrm{J}_{\mathrm{c}}(\mathrm{B}, \mathrm{T})=\mathrm{Jc}(\mathrm{B}, 4.2)(1-(\mathrm{T}-4.2) /(10.7-0.61 \times \mathrm{B}))$.

This formula shows how the critical current density is reduced by temperature or field strength increases

(2) Stress dependence of the critical current density in a multifilamentary $\mathrm{Nb}_{3} \mathrm{Sn}$ conductor.

As seen in Figure 19, the critical current density in a multifilamentary $\mathrm{Nb}_{3} \mathrm{Sn}$ conductor depends strongly on strain. The critical current density peak occurs at the strain which corresponds in magnitude to the compressive prestrain introduced into the surrounding matrix material. The relationship between critical current density and strain is described by the following formula:

$$
J_{c}=J_{c m}(1-a \mid \varepsilon d 1.7) 0.5\left(\left(1-t^{2}\right)\left(1-t^{2}\right)\right)^{2}((1-b)(1-b m))^{2}
$$

where $\varepsilon_{0}=\varepsilon-\varepsilon_{m, t}=T / T c^{*}(\varepsilon), b=B / B c^{*}(T, \varepsilon), T c^{*}$ and $B c^{*}$ are the critical temperature and the critical field of $\mathrm{Nb}_{3} \mathrm{Sn}$ bulk, and $\mathrm{a}$ is 900 for $\varepsilon<0$ and 1,250 for $\varepsilon \geq 0$. 
This section clearly demonstrates how field requirements have a profound repercussion on the choice of superconductors, on the definition of cryostat requirements and structural reguirements. It is clear that all these disciplines play interdependent roles and thus improvements of superconducting magnets can only be made by dealing with these disciplines globally rather than individually. 


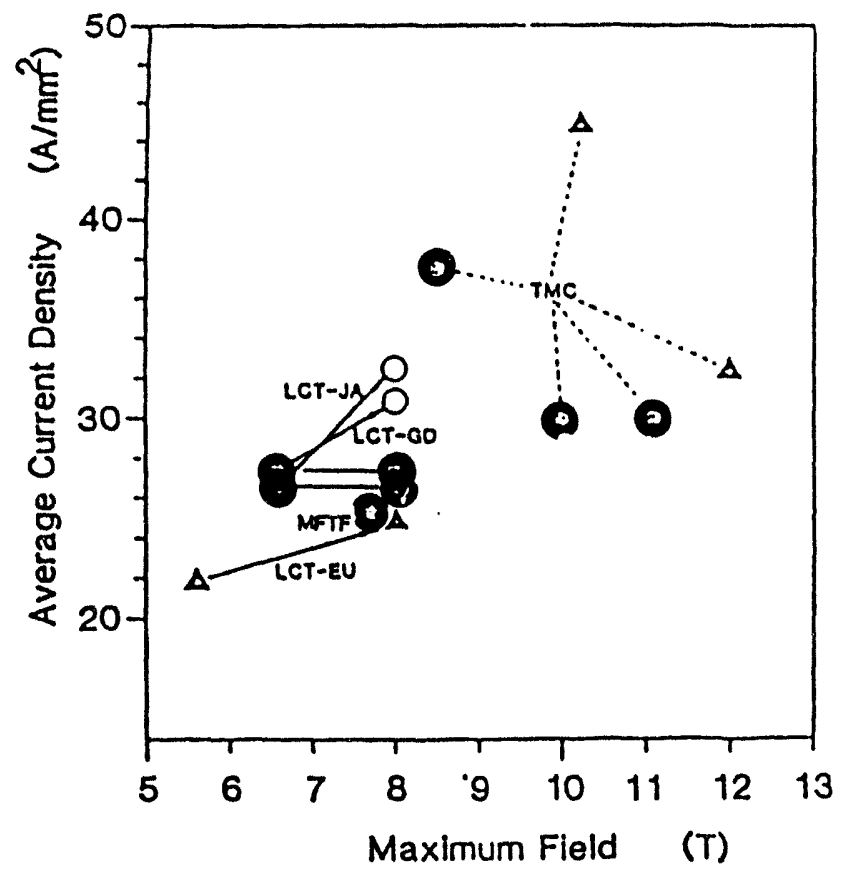

Figure A-2 Average winding current density of large coils already constructed and tested for fusion.

0: already tested and cryostable,

$\Delta:$ already tested without stability test

0 : estimated stability limit.)

(TMD: $12 \mathrm{~T}$ test module coil (Japan), LCT: large coil tests (Akiyama, 1991) 


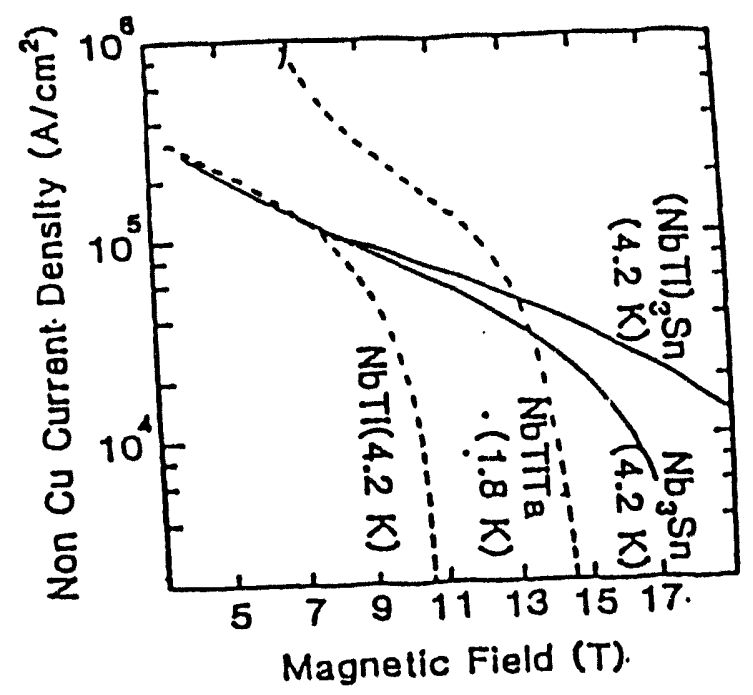

Figure A-3 Critical current density of various materials (Akiyama, 1991) 


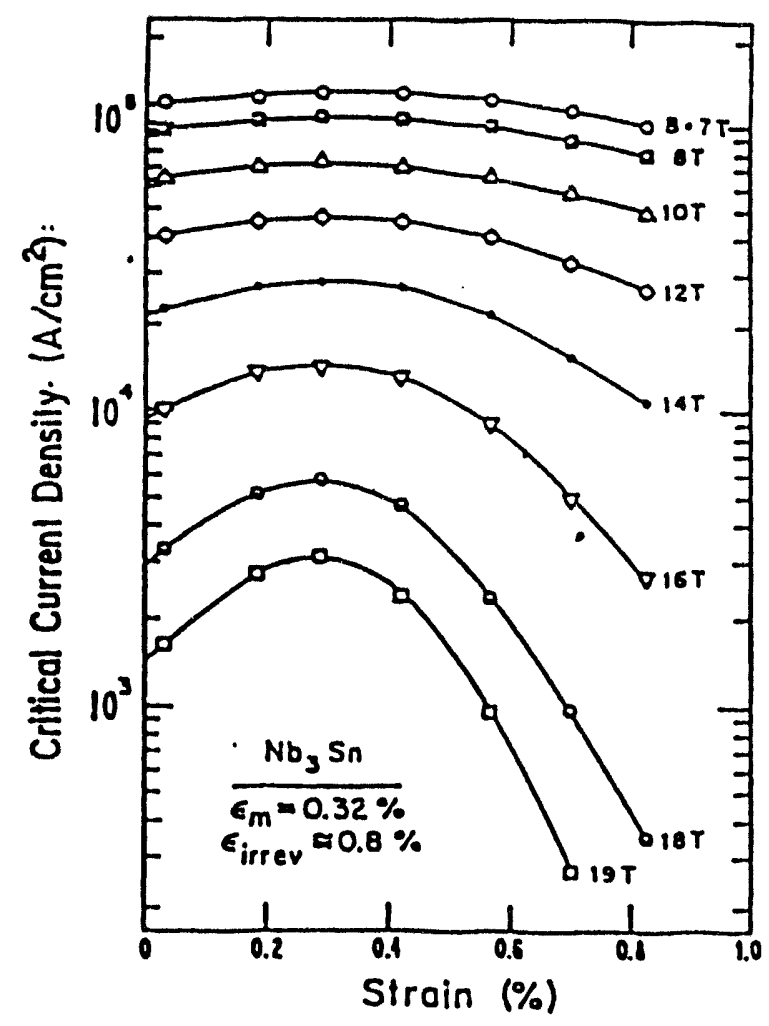

Figure A-4 Strain dependence of the critical current density of $\mathrm{Nb}_{3} \mathrm{Sn}$ (Akiyama, 1991) 


\section{Appendix B \\ Basic Concepts in Maonetostatics}

\section{Biot-Sayart_Lart}

In this Appendix, the basic governing equations in magnetostatics are reviewed and their boundary conditions discussed, because they are important in setting all the parameters required by the TOSCA finite element computer code. A discussion on the possible gauges to close these magnetostatics governing equations is also provided, since they lead to different types of P.D.E. equations to be numerically resolved.

Consider a current carrying wire the magnetic field produced by this wire is given by BiotSavart law which reads:

$$
\mathrm{dB}=\frac{\mu_{0}}{4 \pi R^{2}} J\left(x^{\prime}, y^{\prime}, z\right) \times a_{R} \text { dlds }
$$

where $\mathrm{aR}$, is the unit vector linking the point $\left(x^{\prime}, y^{\prime}, z^{\prime}\right)$ to $(x \cdot y \cdot z)$ and the elementary field $d B$ is calculated at a distance $R$ from the current flowing medium which can be expressed as

$$
R=\left[\left(x-x^{\prime}\right) 2+\left(y-y^{\prime}\right) 2+\left(z-z^{\prime}\right) 2\right]^{12}
$$

The total current is $\mathrm{J}$ ds. The magnetic field due to the current in a volume $\mathrm{v}$ is then:

$$
B(x, y, z)=\frac{\mu_{0}}{4 \pi} \int_{v} \frac{J\left(x^{\prime}, y^{\prime}, z^{\prime}\right) x a_{R}}{R^{2}} d v^{\prime}
$$

Defining a vector potential $A(x, y, z)$ as

$$
A(x, y, z)=\frac{\mu_{0}}{4 \pi} \int_{v} \frac{J\left(x^{\prime}, y^{\prime}, z^{\prime}\right)}{R} d v^{\prime}
$$

and since

$$
\frac{a_{R}}{R^{2}}=-\nabla\left(\frac{1}{R}\right)
$$

then 


$$
B(x, y, z)=\frac{\mu o}{4 \pi} \int_{v} J\left(x^{\prime}, y^{\prime}, z^{\prime}\right) \times \nabla\left(\frac{1}{R}\right) d v^{\prime}
$$

iut

$$
\nabla \times\left(\frac{\mathrm{I}}{\mathrm{R}}\right)=\frac{1}{\mathrm{R}} \nabla \times \mathrm{J}-\mathrm{J} \times \nabla\left(\frac{1}{\mathrm{R}}\right)=-\mathrm{J} \times \nabla\left(\frac{1}{\mathrm{R}}\right)
$$

hence

$$
B(x, y, z)=\nabla \times A
$$

An essential property of the magnetic field is determined by taking the divergence of the magnetic field vector.

$$
\nabla \cdot \mathbf{B}=\nabla \cdot(\nabla \times A)=0
$$

or

$$
\nabla \cdot \mathbf{B}=\mathbf{0}
$$

\section{Boundary Conditions}

Let's consider a boundary between two medi volume element of thickness $h$, surface are: erent physical properties. A coin-shaped a its broad faces parallel to the interface penetrates the boundary and contai ss fortions of both media. We have

$$
n \cdot D_{1}=n \cdot D_{2}
$$

and the normal component of the $\mathrm{D}$ rield is continuous and since

$$
D_{1}=\varepsilon_{1} E_{1} \text { and } D_{2}=\varepsilon_{2} E_{2}
$$

for two general media, then

$$
\frac{n \cdot E_{1}}{n \cdot E_{2}}=\frac{\varepsilon_{2}}{\varepsilon_{1}}
$$

or

$$
\varepsilon_{1} E_{n 1}=\varepsilon_{2} E_{n 2}
$$


and the normal component of the E field is discontinuous when the permitivities are different. Now

$$
\nabla \cdot \mathrm{B}=0
$$

and

$$
\lim _{h \rightarrow 0} \int_{v} \nabla \cdot B d v=\lim _{h \rightarrow 0} \oint_{s} B d s
$$

or

$$
\begin{aligned}
& \left(\mathrm{n} \mathrm{B}_{1}\right) \Delta_{\mathrm{s}}-\left(\mathrm{n} \mathrm{B}_{2}\right) \Delta_{\mathrm{s}}=0 \\
& \mathrm{n} \cdot \mathrm{B}_{1}=\mathrm{n} \cdot \mathrm{B}_{2}
\end{aligned}
$$

and the normal component of the B field is continuous. Using the definitions

$$
\mathrm{B}_{1}=\mu_{1} \mathrm{H}_{1} \text { and } \mathrm{B}_{2}=\mu_{2} \mathrm{H}_{2}
$$

then

$$
\frac{\mathrm{n} \cdot \mathrm{H}_{1}}{\mathrm{n} \cdot \mathrm{H}_{2}}=\frac{\mu_{2}}{\mu_{1}}
$$

or

$$
\mu_{1} H_{n 1}=\mu_{2} H_{n 2}
$$

and the normal component of the $\mathrm{H}$ field is discontinuous when the permeability of the media are different. Also

$$
\nabla \cdot \mathbf{J}=0
$$

and

$$
\lim _{h \rightarrow 0} \int_{v}(\nabla \cdot J) d v=\lim _{h \rightarrow 0} \oint_{s} J \cdot d s=0
$$

and 


$$
\left(n \cdot J_{1}\right) \Delta s-\left(n \cdot J_{2}\right) \Delta s=0
$$

or

$$
\mathrm{n} \cdot \mathrm{J}_{1}=\mathrm{n} \cdot \mathrm{J}_{2}
$$

and the normal component of the current is continuous. With Ohm's law for the two media, i.e.,

$$
\mathrm{J}_{1}=\sigma_{1} \mathrm{E}_{1} \text { and } \mathrm{J}_{2}=\sigma_{2} \mathrm{E}_{2}
$$

then

$$
\frac{\mathrm{n} \cdot \mathrm{E}_{1}}{\mathrm{n} \cdot \mathrm{E}_{2}}=\frac{\sigma_{2}}{\sigma_{1}}
$$

or

$$
\sigma_{1} E_{n 1}=\sigma_{2} E_{n} 2
$$

and the normal component of the $\mathrm{E}$ field is also discontinuous when the electrical conductivities are different. Assuming that the magnetic field is produced by a steady current, then

$$
\nabla \times \mathrm{H}=\mathrm{J}
$$

and

$$
\lim _{b \rightarrow 0} \int_{\Delta s}(\nabla \cdot H) \cdot d s=\lim _{b \rightarrow 0} \oint_{c} H \cdot d l=\lim _{b \rightarrow 0} \int_{\Delta s} J \cdot d s
$$

consequently,

$$
\left(\mathrm{n} \times \mathrm{H}_{1}\right) \Delta \mathrm{l}-\left(\mathrm{n} \times \mathrm{H}_{2}\right) \Delta \mathrm{l}=\mathrm{J}_{\mathrm{S}} \Delta \mathrm{l}
$$

where $\mathrm{J}_{\mathrm{S}}$ is the surface current density. Thus, the jump in the tangential component is due to the surface current, i.e.,

$$
\mathrm{N} \times\left(\mathrm{H}_{1}-\mathrm{H}_{2}\right)=\mathrm{J}_{\mathrm{S}}
$$

In many problems, the surface current is negligibly small, thus, 


$$
\mathrm{n} \times \mathrm{H}_{1}=\mathrm{n} \times \mathrm{H}_{2}
$$

and the tangential component of the $\mathrm{H}$ field is continuous. Again, using the relations, Eq. (2.166),

$$
\frac{\mathrm{n} \times \mathrm{B}_{1}}{\mathrm{n} \times \mathrm{B}_{2}}=\frac{\mu_{1}}{\mu_{2}}
$$

or

$$
\mu_{2} \mathrm{~B}_{\tau 1}=\mu_{1} \mathrm{~B}_{\tau 2}
$$

and the tangential component of the B field is discontinuous when the permeability's are different.

\section{Magnetostatics Governing_Equations:}

The quasi-static electromagnetic field governing equations at low frequency can be represented by:

$$
\begin{aligned}
\nabla \cdot \mathrm{D}=\rho & \text { (Gauss's Law) } \\
\nabla \cdot \mathrm{B}=0 & \\
\nabla \times \mathrm{E}=\frac{\partial \mathrm{B}}{\partial \mathrm{t}} & \text { (Faraday's Law) } \\
\nabla \times \mathrm{H}=\mathrm{J} & \text { (Ampere's Law) }
\end{aligned}
$$

where $\mathrm{D}, \mathrm{B}, \mathrm{E}, \mathrm{H}$ are the usual field vectors, $\rho$ and $\mathrm{J}$ the free charge and current densities respectively. The field vectors are not independent since they are further related by the material constitutive properties (see also previous section);

$$
\begin{aligned}
& D=\varepsilon E \\
& B=\mu H
\end{aligned}
$$

where $\varepsilon$ and $\mu$ are the material permitivity and permeability respectively. The current density in a conductor moving with relative velocity $v$ is generated by the Lorentz force and is given by:

$$
\mathrm{J}=\sigma(\mathrm{E}+\mathrm{v} \times \mathrm{B})(\text { Ohm's Law) }
$$

where $\sigma$ is the material conductivity. In practice $\mu$ and $\sigma$ may often be field dependent quantities, and furthermore, some materials will exhibit both anisotropic and hysteretic effects. The current continuity condition is: 


$$
\nabla \cdot \mathbf{J}=0
$$

The four field vectors must satisfy the following conditions at the interfaces between regions of different material properties;

$$
\begin{aligned}
& \left(B_{2}-B_{1}\right) \cdot n=0 \\
& \left(D_{2}-D_{1}\right) \cdot n=\omega \\
& \left(H_{2}-H_{1}\right) \times n=K \\
& \left(E_{2}-E_{1}\right) \times n=0
\end{aligned}
$$

where $\mathrm{K}$ and $\omega$ are the surface current and charge densities respectively. These relations follow directly from the limiting forms of field equations (see previous section).

\section{Magnetic Yector Potential}

Since the field vector B satisfies a zero divergence condition, it can be expressed in terms of a vector potential $\mathrm{A}$ as follows:

$$
\mathrm{B}=\nabla \times \mathrm{A}
$$

and then, it follows that,

$$
\nabla \times\left(E+\frac{\partial}{\partial t} A\right)=0
$$

and hence by integrating to give,

$$
E=-\left(\frac{\partial}{\partial t} A+\nabla V\right)
$$

where $\mathrm{V}$ is a scalar potential. Neither $\mathrm{A}$ nor $\mathrm{V}$ are completely defined since the gradient of an arbitrary scalar function can be added to $A$ and the time derivative of the same function can be subtracted from $V$ without affecting the physical quantities $E$ and $B$. These changes to $A$ and $V$ are the so called gauge transformations, and uniqueness is usually ensured by specifying the divergence (gauge) of $\mathrm{A}$ and sufficient boundary conditions. Thus, the field equations in terms of $A$ and $V$ are as follows: 


$$
\begin{aligned}
& \nabla \times \mathrm{xH}=\nabla \times \frac{1}{\mu} \nabla \mathrm{xA}+\emptyset\left(\frac{\partial \mathrm{A}}{\partial \mathrm{t}}+\nabla \mathrm{V}\right)=\mathrm{J}_{s} \\
& \nabla \cdot \mathrm{B}=\nabla \cdot \sigma\left(\frac{\partial \mathrm{A}}{\partial \mathrm{t}}+\nabla \mathrm{V}\right)=0
\end{aligned}
$$

where $\mathrm{J}_{\mathrm{S}}$ is the current source density. In the above expressions, eddy currents are expressed by

$$
\oint\left(\frac{\partial A}{\partial t}+\nabla V\right)
$$

In the region, where no conductors are present $(\sigma=0)$, one obtains

$$
\nabla \times \mathrm{xH}_{\mathbf{S}}
$$

Since $\nabla \cdot B=0$, one can define a magnetic scalar potential, $\phi$, such that

$$
\mathrm{B}=\mu \nabla \phi \text { with }(\mathrm{H}=\nabla \phi)
$$

with

$$
\nabla \cdot(\mu \nabla \phi)=0
$$

\section{Coulomb - Lorentz Gauges}

To ensure uniqueness of the Magnetic Vector Potential and of Electric potential, two gauges, the Coulomb and the Lorentz gauges, are generally used to close system of equations.

\section{(a) Coulomb Gauge}

For the Coulomb gauge, we set $\nabla \cdot A=0$. The governing equations for $A$, can be re-written as

$$
\nabla \times \frac{1}{\mu} \nabla x A-\nabla \frac{1}{\mu} \nabla \cdot A=-\oint\left(\frac{\partial}{\partial t} A+\nabla V\right)
$$

with

$$
\nabla \cdot \sigma\left(\frac{\partial \mathrm{A}}{\partial t}\right)+\nabla \cdot \sigma \nabla V=0
$$


which leads to the uncoupled equation in A:

$$
\nabla^{2}\left(\frac{1}{\mu} \nabla \cdot A\right)=0
$$

\section{(b) Lorentz Gauge}

The governing equations for $\mathrm{A}$ with no gauge applied are re-written as

$$
\nabla \times \frac{1}{\mu} \nabla x A=-\sigma\left(\frac{\partial}{\partial t} A+\nabla V\right)
$$

and

$$
\nabla \cdot \sigma \frac{\partial \mathrm{A}}{\partial \mathrm{t}}+\nabla \cdot \sigma \nabla \mathrm{V}=0
$$

As can be seen these two equations are not independent. The Lorentz Gauge condition can now be used to obtain a sufficient set of defining equations with $\mathrm{A}$ and $\mathrm{V}$ defined independently. The low frequency form of the Lorentz gauge is,

$$
\nabla \cdot \mathrm{A}=-\mu \sigma \mathrm{V}
$$

Combining these equations leads to:

$$
\nabla \times \frac{1}{\mu} \nabla \times A=-\sigma \frac{\partial A}{\partial t}+\sigma \nabla\left(\frac{1}{\mu \sigma} \nabla \cdot A\right)
$$

and

$$
-\mu \sigma^{2} \frac{\partial V}{\partial t}+\nabla \cdot \sigma \nabla V=0
$$

where $\sigma$ is assumed piece wise constant. Thus this approach reflects the classical motivation for the Lorentz Gauge namely, to decouple the vector potential from the scalar potential. 


\section{References}

Akiyama, M., Design Technology of Fusion Reactors, World Scientific, Singapore, (1991).

Akiyama, M., Design Technology of Fusion Reactors, Vol. 6, Series in Theoretical and Applied Mechanics, edited by R.K.T. Hsieh (1991).

ANSYS: Swanson Analysis Systems, Inc., Houston, PA, 15342-0065.

Bobrova, W.A. and S.I. Syrovatskii, Singular Lines of One-Dimensional Force-Free Magnetic Fields, Sol. Phys., 61, 379 (1979).

Buck, G.J., Force-Free Magnetic Field Solution in Toroidal Coordinates, Journal of Applied Physics, Vol. 36, No. 7, pp. 2231-2235 (1965).

Campbell, L.J., and J.B. Kadtke, Stationary Configurations of Point Vortices and Other Logarithmic Objects in Two-Dimensions, Physical Review Letters, Vol. 58, No. 7, pp. 670-673 (1987).

Chapman, M.S., J.M. Cortella, I. Schermer, and R.H. Wanas, Mechanical Analysis of Different Yoke Configurations for SCC Dipole, pp. 85-90, 77th International Conference on Magnet Technology, Edited by T. Sekiguthi and S. Shimamoto, Vol. 1, Tsukuba, Japan, Elsevier Applied Science (1989).

Cody, G.D., G.W,.Cullen, and J.P. McEvoy, Field and Angular Dependence of Critical Currents in Nb3SnII; Review of Modern Magnets, pp. 95-97 (January 1964).

Furth, H.P., S.C. Jardin and D.B. Montgomery, IEEE Trans Magn. 24, 1467 (1988).

Furth, H.P., M.A. Levine, and R.W. Waniek, Production and Use of High Transient Magnetic Fields, Review of Scientific Instruments, Vol. 28, No. 11, pp. 349-958 (1957).

Hanksworth, D., Development of Superconducting Magnet Systems for MRI, Advances in Cryogenic Engineering, Vol. 35, pp. 525-538, edited by R.W. Fast, Plenum Press, New York (1990).

Kadtke, J.B. and L.J. Campbell, Method for Finding Stationary States of Point Vortices, Physical Review A, Vol. 36, No. 9, pp. 4360-4370 (1987).

Lundquist, S., Magneto-Hydrostatic Fields, Arh. Fys. 2, 361 (1951).

Marsh, G.E., A Class of Cylindrically Symmetric Solutions to the Force-Free Magnetic Field Equations With Nonccnstant $\alpha$, J. Appl. Phys. Vol. 68, No. 8, pp. 3818-3821 (1990).

Marsh, G.E., Axially Symmetric Solutions to the Force-Free Magnetic Field Equations in Spherical and Eylindrical Coordinates, Physical Review A, Vol. 45, No. 10, pp. 7520-7525 (1992).

Mawardi, O.K.., Design of Force-Free Inductive Coil, Los Alamos Report LA-5953-MS, (June 1975). 
Moon, F.C., The Virial Theorem and Scaling, Laws for Superconducting Magnet Systems, J. Appl. Phys. 53(12), pp. $9112-9121$ (1982).

MOSES, R.W., JR., Configurational Design of Superconductive Energy Storage Magnets, pp. 140-148, in Advances in Cryogenic Engineering, Vol: 21, edited by K.O. Timerhaus and D.H. Weitzel (1975).

Prueitt, M.L., F.M. Mueller, and J.L. Smith, Apparatus for Storing High Magnetic Fields Having Reduced Mechanical Forces and Reduced Magnetic Pollution, Patent No. 5,006,672, April 9, 1991.

Prueitt, M.L., F.M. Mueller, and J.L. Smith, Apparatus Having Induced Mechanical Force-Free Supporting High Magnetic Fields, Patent No. 4,992,696, Feb. 12, 1991.

Thome, R.J., Nims and Fusion Magnets: Field and Force Design Concepts, John Wiley and Sons (1982).

Tsujii, Y., Force Free Magnetic Field in the Axisymmetric Torus of Arbitrary Aspect Ratio, Phys. Fluids B, Vol. 3, No. 12, pp. 3379-3387 (1991).

TOSCA Reference Manual; Vector Fields, Aurora, II.

Wells, D.R. and R.C. Mills, High Magnetic Fields, Proceedings of the International Conference on High Magnetic Fields, November 1-4, 1961, edited by H. Kolm, B. Lax, F. Bitter and R. Mills. 
$r$
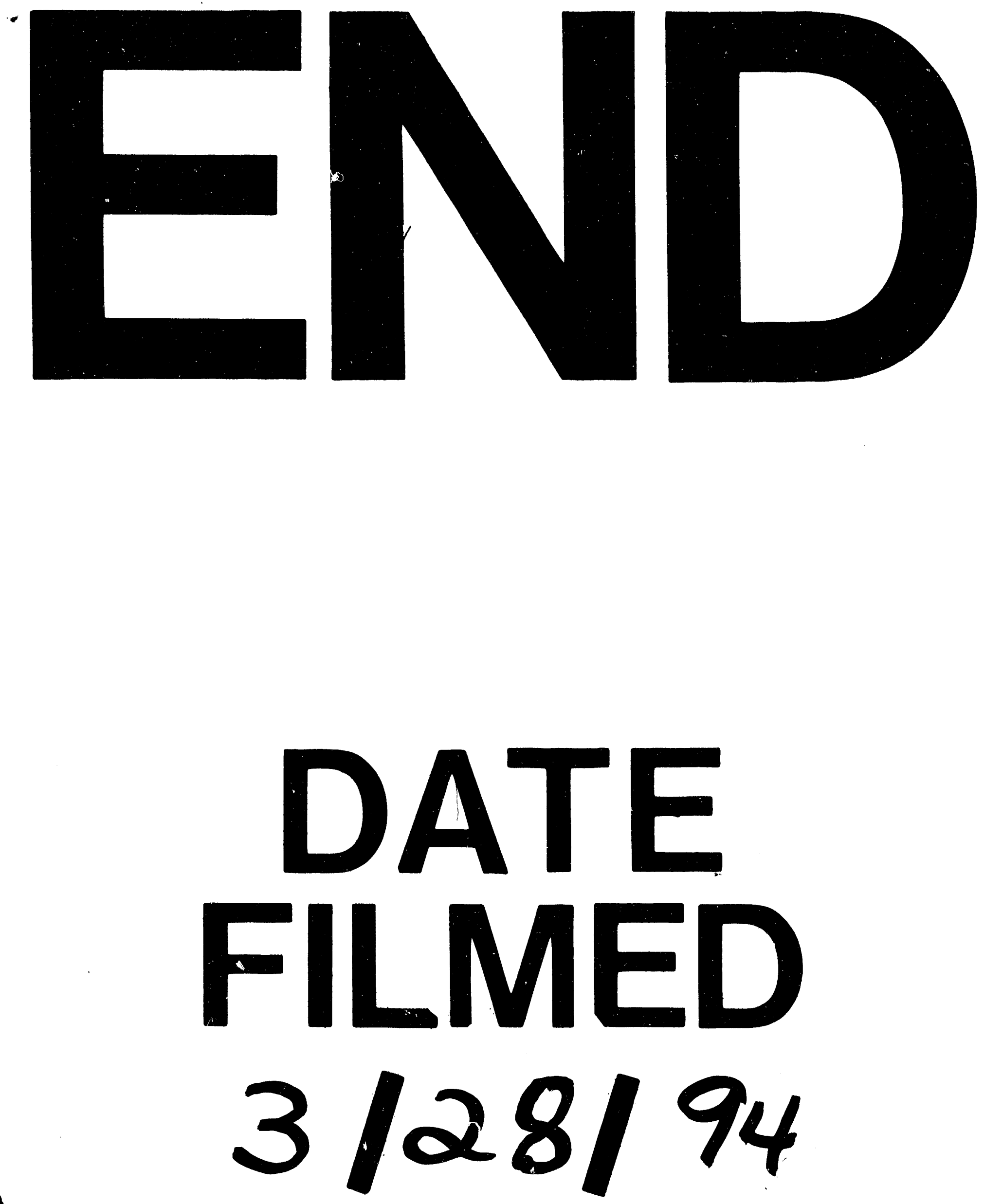

1 
